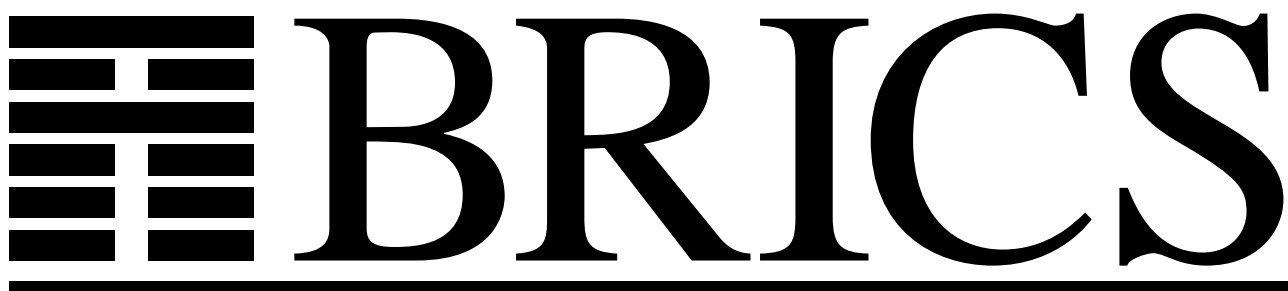

Basic Research in Computer Science

\title{
Profunctors, Open Maps and Bisimulation
}

Gian Luca Cattani

Glynn Winskel

RS-04-22 
Copyright (c) 2004, Gian Luca Cattani \& Glynn Winskel. BRICS, Department of Computer Science University of Aarhus. All rights reserved.

Reproduction of all or part of this work is permitted for educational or research use on condition that this copyright notice is included in any copy.

See back inner page for a list of recent BRICS Report Series publications. Copies may be obtained by contacting:

\author{
BRICS \\ Department of Computer Science \\ University of Aarhus \\ Ny Munkegade, building 540 \\ DK-8000 Aarhus C \\ Denmark \\ Telephone: +4589423360 \\ Telefax: $\quad+4589423255$ \\ Internet: BRICS@brics.dk
}

BRICS publications are in general accessible through the World Wide Web and anonymous FTP through these URLs:

http://www.brics.dk

ftp: //ftp.brics.dk

This document in subdirectory RS / 04 / 22 / 


\title{
Profunctors, Open Maps and Bisimulation*
}

\author{
Gian Luca Cattani \\ DS Data Systems S.p.A., \\ Via Ugozzolo 121/A, \\ I-43100 Parma, Italy. \\ Email: cattanil@dsdata.it. \\ Glynn Winskel \\ University of Cambridge Computer Laboratory, \\ Cambridge CB3 0FD, England. \\ Email: gw104@cl.cam.ac.uk.
}

October 2004

\begin{abstract}
This paper studies fundamental connections between profunctors (i.e., distributors, or bimodules), open maps and bisimulation. In particular, it proves that a colimit preserving functor between presheaf categories (corresponding to a profunctor) preserves open maps and open map bisimulation. Consequently, the composition of profunctors preserves open maps as 2-cells. A guiding idea is the view that profunctors, and colimit preserving functors, are linear maps in a model of classical linear logic. But profunctors, and colimit preserving functors, as linear maps, are too restrictive for many applications. This leads to a study of a range of pseudo-comonads and how non-linear maps in their co-Kleisli bicategories preserve open maps and bisimulation. The pseudo-comonads considered are based on finite colimit completion, "lifting", and indexed families. The paper includes an appendix summarising the key results on coends, left Kan extensions and the preservation of colimits. One motivation for this work is that it provides a mathematical framework for extending domain theory and denotational semantics of programming languages to the more intricate models, languages and equivalences found in concurrent computation. But the results are likely to have more general applicability because of the ubiquitous nature of profunctors.
\end{abstract}

\section{Contents}

1 Introduction 3

2 Presheaves, open maps and bisimulation 6

*To appear in Mathematical Structures in Computer Science 
3 A result on open map preservation $\quad 9$

4 The bicategory Prof and the 2-category Cocont 14

5 The structure of Prof $\quad 17$

5.1 Pseudo-products and -coproducts . . . . . . . . . . . . . . 17

5.2 Tensor . . . . . . . . . . . . . . . . . . . . . . 18

5.3 Dualiser .......................... . . . . . . . . . . . . . . .

5.4 Function space . . . . . . . . . . . . . . . . . . . . . . . . . . . . . . . . . . . .

5.5 Linear logic . . . . . . . . . . . . . . . . . . . . 20

6 Open map bisimulation in Prof 21

7 Prof and $\omega$-accessible categories 24

$7.1 \omega$-Accessible categories . . . . . . . . . . . . . . . . . 24

7.2 Finite colimit completion . . . . . . . . . . . . . . . . 26

7.2.1 Domain theoretic analogies . . . . . . . . . . . . . . . . . 31

7.3 A failure of open map preservation . . . . . . . . . . . . . . . 31

8 Lifting and connected colimits $\quad 32$

8.1 Lifting . . . . . . . . . . . . . . . . . . . . . . . . . . . . . . . . . .

8.2 Connected colimit preserving functors . . . . . . . . . . . . . . 34

8.2.1 Rooted colimits . . . . . . . . . . . . . . 37

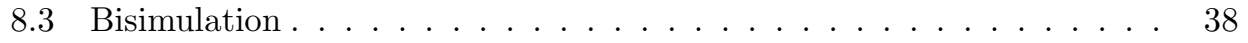

9 Pseudo comonads via families $\quad 40$

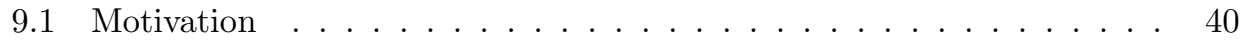

9.2 Indexed families . . . . . . . . . . . . . . . . . . . . . . . . . . . . . . . . . . . .

9.3 Pseudo-comonads on Prof _. . . . . . . . . . . . . . . . . . . 42

9.4 On preservation of bisimulation . . . . . . . . . . . . . . . 43

10 Conclusions $\quad 48$

A A primer on coends and left Kan extensions $\quad \mathbf{5 0}$

A.1 Representations, universality and parametricity . . . . . . . . . . . 50

A.2 (Co)Ends and their properties . . . . . . . . . . . . . . 51

A.2.1 Dinatural transformations . . . . . . . . . . . . 51

A.2.2 Coends . . . . . . . . . . . . . . . . . 51

A.2.3 Parametricity for coends . . . . . . . . . . . . . . . 53

A.2.4 The Fubini theorem for coends . . . . . . . . . . . . . . 54

A.2.5 Ends . . . . . . . . . . . . . . . . . . 55

A.2.6 End and coend formulae . . . . . . . . . . . . 55

A.3 Preservation of colimits . . . . . . . . . . . . . . . . . 55

A.4 Kan extensions and their properties . . . . . . . . . . . . . 56

A.4.1 Left Kan extensions . . . . . . . . . . . . . . . . . . . 56

A.4.2 Pointwise left Kan extensions . . . . . . . . . . . . . . . . 57

A.4.3 Left Kan extensions along Yoneda . . . . . . . . . . . . . . . 58

A.4.4 The density formulae . . . . . . . . . . . . . . . . 59

A.4.5 A factorisation lemma ............... 59 


\section{Introduction}

At first sight, it is perhaps surprising that profunctors, ${ }^{1}$ a categorical generalisation of relations [5, 31], and bisimulation [37, 42], a central equivalence in the study of processes are intimately related. Briefly, the chain of connections runs:

- Nondeterministic processes can be represented as presheaves. A presheaf over a category $\mathbb{P}$ can be thought of as a form of transition system whose computation paths have shapes objects in $\mathbb{P}$; the objects of $\mathbb{P}$ are paths and the arrows of $\mathbb{P}$ express how one computation path can extend to another. A presheaf category $\widehat{\mathbb{P}}=\left[\mathbb{P}^{o p}\right.$, Set $]$ is the free colimit completion of $\mathbb{P}$, so its objects, presheaves, as colimits, are collections of paths identified along subpaths. Familiar models of processes such as known categories of synchronisation trees and event structures, and many others, can be realised as presheaf categories $\mathbb{P}$ for some suitable choice of category $\mathbb{P}[28]$.

- Bisimulation between processes is caught via spans of open maps. An open map between presheaves is a generalisation of a functional bismulation between transition systems (i.e., a bisimulation whose underlying relation on states happens to be a function). In many, though not all, cases the bisimulation obtained coincides with familiar definitions [28].

- Profunctors correspond to colimit preserving functors between presheaf categories, which somewhat remarkably preserve open maps and so bisimulation (see Theorem 3.3).

The concept of a bisimulation was invented by Milner and Park as a relation between the states of labelled transition systems to express when two states have essentially the same communication capabilities [37, 42]. Showing processes bisimilar (an equivalence given as a maximum fixed point) amounts to exhibiting a bisimulation (a postfixed point) relating them. This coinductive method comes from a direct reading of Tarski's fixed point theorem [50].

Subsequently the idea of bisimulation has been extended and generalised to a range of languages and models, most often based on a transition system obtained from an operational semantics. Though a pattern has emerged, bisimulation is most often defined in an ad hoc manner for the language at hand, and sometimes can be a matter of great subtlety [36].

Broadly speaking, there are two lines of development in making the definition of bisimulation more systematic; so that the variety of bisimulation is determined by the denotational semantics given to a language. One approach is that based on the recognition that bisimulation arises from final coalgebras. This line is very fruitful in a range of categories of process models and domain theories, and often furnishes useful proof principles of coinduction, echoing the technique promoted by Milner and Park [25]. The other approach is based on open maps.

Open maps have a prehistory in pure mathematics [27], but first appeared in computer science in [28]. Their initial role was in giving a unified approach to a

\footnotetext{
${ }^{1}$ Also called distributors and bimodules.
} 
range of models for concurrent computation, from interleaving models like transition systems to independence, or causal, models such as Petri nets and event structures. As summarised in the handbook chapter $[54,55]$, it had become useful to regard models for concurrency as categories (for example, as a category of transition systems, or a category of Petri nets). Then the constructions being used to model processes in a variety of models could be understood in a uniform way, as being the same categorical constructions, and different models were often related by adjunctions. The diagrammatic definition of open maps, expressing a path-lifting property, made sense in a range of categories of models for concurrency.

The landscape of models was however somewhat arbitrary and patchy. The categories of traditional models were not sufficient in themselves to provide semantics to higher order processes, or even CCS with late value passing. The fact that open maps were based on paths suggested building models for processes directly on the computation paths of which the processes were capable. Given a category of computation paths $\mathbb{P}$, the presheaf category $\left[\mathbb{P}^{o p}\right.$, Set $]$ is its colimit completion. An individual presheaf $X: \mathbb{P}^{\text {op }} \rightarrow$ Set consists of a collection of computation paths glued together at the shared subpaths, from which they nondeterministically branch.

Presheaf categories fill in the landscape of models to provide a range of models for concurrency. They are as versatile as the notion of computation path. With suitable choices of computation path, presheaves subsume traditional models such as synchronisation trees (where paths are finite sequences) and event structures (where paths are finite partial orders of events). (This is one place where a traditional use of powerdomains based on domains of resumptions [43], can fall short; being based on a nondeterministic choice of actions one at a time, it cannot accommodate the potentially complex structure of computation paths.)

Profunctors are maps relating presheaf categories. As such, profunctors can play a fundamental role in understanding the semantics of interacting processes, and suggest a new form of domain theory for concurrency. According to this view, objects of the bicategory of profunctors Prof, which are small categories $\mathbb{P}, \mathbb{Q}, \cdots$, stand for types of processes. A process having type $\mathbb{P}$ means that the process performs computation paths which lie in $\mathbb{P}$. The arrows of Prof, are profunctors $F: \mathbb{P} \rightarrow \rightarrow \mathbb{Q}$ and so functors $F: \mathbb{P} \times \mathbb{Q}^{\text {op }} \rightarrow$ Set, and so correspond to functors $\bar{F}: \mathbb{P} \rightarrow \widehat{\mathbb{Q}}$. Because presheaf categories are free colimit completions, this means that profunctors from $\mathbb{P}$ to $\mathbb{Q}$ correspond to colimit preserving functors between presheaf categories from $\widehat{\mathbb{P}}$ to $\widehat{\mathbb{Q}}$, and map processes of type $\mathbb{P}$ to processes of type $\mathbb{Q}$. The bicategory Prof can be endowed with a rich type discipline guided by the view of Prof as a model of classical linear logic. In particular, there are function spaces $\mathbb{P} \multimap \mathbb{Q}$, the type of higher order processes which take a process of type $\mathbb{P}$ as argument and deliver a process of type $\mathbb{Q}$ as result. Recursive domain equations can also be treated in this generalised setting [12].

It is sensible to view a profunctor $F: \mathbb{P} \rightarrow \mathbb{Q}$ as a linear map which on input of a process of type $\mathbb{P}$ yields a process of type $\mathbb{Q}$. Linearity is about how to manage without a presumed ability to copy or discard, and accordingly a linear map uses exactly one copy of the input process. Although it can be hard or impossible for processes to copy processes, which may be highly distributed, it is generally easy for processes to ignore other processes. So, for many applications linearity is too stringent a general requirement on maps. For example, a profunctor, regarded as a colimit preserving functor between presheaf categories, will necessarily send the empty presheaf to the empty presheaf; input of the inactive nil process will always yield the nil process. In 
linear logic the standard way around this stringency is to take maps from $\mathbb{P}$ to $\mathbb{Q}$ to be linear maps from $\mathcal{F}(\mathbb{P})$ to $\mathbb{Q}$ where $\mathcal{F}$ is an operation on types obeying laws including those of a comonad. A choice of $\mathcal{F}$ which allows input to be discarded but not copied will lead to affine maps, while other choices can support various regimes of copying. This methodology can be followed for profunctors when different choices of $\mathcal{F}$ determine maps which are linear/affine/continuous according to whether they use (exactly one)/(at most one)/(finitely many) copies of the input process.

Whether a map is linear/affine/continuous is reflected in whether a path of its output is determined by (exactly one)/(at most one)/(finitely many) paths of the input process. Accordingly an object of $\mathcal{F}(\mathbb{P})$ can be thought of as a form of compound path consisting of an assembly of paths (i.e., objects) of $\mathbb{P}$. One interesting case we shall study is when $\mathcal{F}(\mathbb{P})$ is $\mathbb{P}_{\perp}$ consisting of $\mathbb{P}$ to which an initial empty path has been freely adjoined. From this choice we obtain a form of affine linear map, and accordingly a model of affine linear logic. Another interesting case is when $\mathcal{F}(\mathbb{P})$ is the free finite colimit completion of $\mathbb{P}$. An object of $\mathcal{F}(\mathbb{P})$ can then be thought of as a finite collection of paths, objects from $\mathbb{P}$, glued together along subpaths. The associated (continuous) maps correspond to filtered colimit preserving functors between presheaf categories; the category is cartesian closed, and a model of intuitionistic logic. This example is fairly well known. But, as we shall see, there are several other interesting possible choices for $\mathcal{F}$, and they can behave better with respect to open maps.

Linearity underpins distributed processes. Although we cannot expect all maps to be linear, it is useful when they are (linear maps preserve colimits so nondeterministic sums) and, in the standard fashion, we can moderate the strictness of linearity by explicitly allowing the discarding and copying of processes. The bicategory of profunctors is one place where all this can be made precise, ${ }^{2}$ while at the same time being rich enough in structure to subsume a range of models and support bisimulation. The references, especially in "Conclusions", provide the beginnings of a bibliography of its applications to the semantics of process languages.

A remark on applications and examples. Where appropriate we point to applications to process models and the semantics and equivalences of existing of process languages. To a large extent the mathematics has been developed in order to interpret processes as presheaves. But we don't see our primary business as being in chasing up the latest process syntax to give it mathematical meaning. The mathematics has a curious life of its own, exhibiting much more structure than is currently reflected in process languages. A role of the mathematics is to suggest new connections and insights, as well as new process languages and models, operational semantics and equivalences.

\section{Outline}

We start in Section 2 by recalling the fundamental definitions and properties of presheaf categories, open maps and bisimulation, including preservation properties of open maps across adjunctions, for later use. Section 3 is devoted to the proof of

\footnotetext{
${ }^{2}$ Another place is in the work of Matthew Hennessy[20], who in developing a domain theory for concurrency used a direct analogue of Prof, essentially one based on relations $F: \mathbb{P} \times \mathbb{Q}^{\text {op }} \rightarrow \mathbf{2}$ where the role of the category Set in defining a profunctor has been replaced by the partial order comprising $0<1$. See too the more recent work of Nygaard and Winskel on a domain theory for concurrency based on this view [40]. A semantics based on such relations is not sufficiently sensitive to the branching behaviour of processes to support bisimulation.
} 
a major result: that colimit preserving functors between presheaf categories preserve open maps. In Section 4 the bicategory of profunctors Prof is introduced alongside the equivalent 2-category in which arrows are colimit preserving functors between presheaf categories. Section 5 exhibits the rich structure of the bicategory Prof, explaining the sense in which it can be made into a model of classical linear logic, once a choice of (pseudo) comonad for the exponential is made. The result on preservation of open maps in Section 3 is extended to preservation results for Prof in Section 6, showing that composition of profunctors preserves open maps. Our first candidate for an exponential on Prof is motivated by an analogy with domain theory. This analogy is pursued in Section 7; the construction of forming a presheaf category is shown analogous to a powerdomain, and the bicategory of profunctors analogous to a category of nondeterministic domains. The continuous maps induced between presheaf categories do not preserve open maps and bisimulation in general. So in Sections 8 and 9 we look more broadly at other ways in which to moderate the linear maps that are profunctors to obtain affine and continuous maps suitable for denotational semantics. This can be achieved in a uniform way via pseudo-comonads based on families of paths, with results emphasising the preservation of open maps. The Conclusion points to the current status of presheaf models for concurrency, which is one of the major application areas.

Finally, some remarks on category theory. We rely heavily on coend notation and left Kan extensions, the main results concerning which are summarised in the Appendix, along with further references. It is extremely helpful to make use of naturality to simplify proofs that functors expressed as coends preserve colimits - see Section A.3. The results of the Appendix are perhaps best referred to in a demand driven way. We have tried to be as light handed as possible in our treatment of 2-categorical and bicategorical issues. The use in this paper of pseudo-comonads predated and to some extent motivated Cheng, Hyland and Power's systematic definition and study of pseudo monads and their attendant constructions [16]. We refer the reader to that work and the recent work of Power and Tanaka [46, 49] for the definitions and results of pseudo-monads and pseudo-comonads on a 2-category, and to legitimise the terminology here. We will use their concepts for bicategories, as they transfer via biequivalences of the bicategories with specific 2-categories. Finally, the reader is warned that for us a category being small means that it is equivalent to a category of which the objects and arrows form sets (what others often call "essentially small").

\section{Presheaves, open maps and bisimulation}

In this section we recall the definition and main properties of presheaf categories. We introduce the definition of bisimulation on presheaves via open maps. The original motivation for viewing processes as presheaves and basic results can be found in [28].

Let $\mathbb{P}$ be a small category. The category of presheaves over $\mathbb{P}$, often denoted by $\widehat{\mathbb{P}}$ or by Set $^{\mathbb{P}^{\text {op }}}$, is the functor category $\left[\mathbb{P}^{\mathrm{op}}\right.$, Set $]$ whose objects are contravariant functors from $\mathbb{P}$ to Set (the category of sets and functions) and whose arrows are the natural transformations between such functors.

A category of presheaves, $\widehat{\mathbb{P}}$, is accompanied by the Yoneda embedding, a functor $\mathrm{y}_{\mathbb{P}}: \mathbb{P} \rightarrow \widehat{\mathbb{P}}$, which fully and faithfully embeds $\mathbb{P}$ in the category of presheaves. For 
every object $P$ of $\mathbb{P}$, the Yoneda embedding yields $\mathrm{y}_{\mathbb{P}}(P)=\mathbb{P}(-, P)$. Presheaves isomorphic to images of objects of $\mathbb{P}$ under the Yoneda embedding are called representables.

Via the Yoneda embedding we can regard $\mathbb{P}$ essentially as a full subcategory of $\widehat{\mathbb{P}}$. Moreover $\widehat{\mathbb{P}}$ is characterized (up to equivalence) as the free colimit completion of $\mathbb{P}$. In other words, the Yoneda embedding $\mathrm{y}_{\mathbb{P}}$ satisfies the universal property that for any functor $F: \mathbb{P} \rightarrow \mathcal{E}$, where $\mathcal{E}$ is a cocomplete category, there is a colimit preserving functor $G: \widehat{\mathbb{P}} \rightarrow \mathcal{E}$, determined to within isomorphism such that $F \cong G \circ y_{\mathbb{P}}$ :

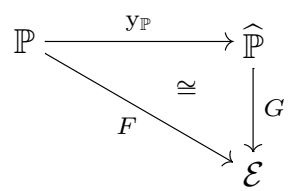

We may choose $G$ such that $F=G \circ y_{\mathbb{P}}$. Observe that $G$ is the functor part of the left Kan extension of $F$ along $\mathrm{y}_{\mathbb{P}}, \operatorname{Lan}_{\mathrm{yP}}(F)$ - see Appendix A.4.3. Notice also that the functor $\operatorname{Lan}_{\mathrm{yP}}(F)$ above always has a right adjoint, $F^{*}: \mathcal{E} \rightarrow \widehat{\mathbb{P}}$ given by $F^{*}(E)=\mathcal{E}(F(-), E)$.

In applications to the semantics of concurrent processes, the category $\mathbb{P}$ is to be thought of as consisting of path objects, or computation-path shapes. The Yoneda Lemma [33], by providing a natural bijection between $\widehat{\mathbb{P}}\left(y_{\mathbb{P}}(P), X\right)$ and $X(P)$, justifies the intuition that a presheaf $X: \mathbb{P}^{\circ p} \rightarrow$ Set can be thought of as specifying for a typical path object $P$ the set $X(P)$ of computation paths of shape $P$. The presheaf $X$ acts on a morphism $m: P \rightarrow Q$ in $\mathbb{P}$ to give a function $X m: X(Q) \rightarrow X(P)$ saying how $Q$-paths restrict to $P$-paths. A presheaf being a colimit of path objects can be thought of as a collection of computation paths glued together by identifying sub-paths.

Bisimulation on presheaves is derived from the notion of open map [27].

Definition 2.1 A morphism $f: X \rightarrow Y$, between presheaves $X, Y$, is $\mathbb{P}$-open if for all morphisms $m: P \rightarrow Q$ in $\mathbb{P}$, the square of functions

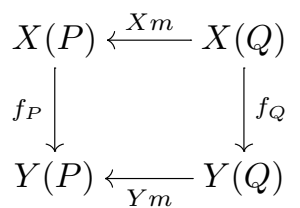

is a quasi-pullback, i.e. whenever $x \in X(P)$ and $y \in Y(Q)$ satisfy $f_{P}(x)=(Y m)(y)$, then there exists $x^{\prime} \in X(Q)$ such that $(X m)\left(x^{\prime}\right)=x$ and $f_{Q}\left(x^{\prime}\right)=y$.

In [28] a broader notion of open map, based on a path lifting property was presented:

Definition 2.2 Let $\mathcal{M}$ be a category and $I: \mathbb{P} \rightarrow \mathcal{M}$ a functor. Say that an arrow $f: M_{1} \rightarrow M_{2}$ is I-open if for every commuting square

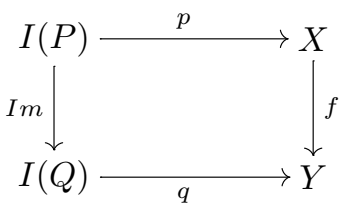


there exists an arrow $r: I(Q) \rightarrow X$ such that $r(\operatorname{Im})=p$ and $f r=q$.

Let $I: \mathbb{P} \rightarrow \mathcal{M}$. Note that any isomorphism is $I$-open and that $I$-open maps form a subcategory. Another useful, direct consequence of the definition of openness is the following. Suppose $I^{\prime}: \mathbb{P}^{\prime} \rightarrow \mathcal{M}$ and that $I^{\prime}$ factors through $I$ in the sense that $I^{\prime} \cong I \circ J$ for some functor $J: \mathbb{P}^{\prime} \rightarrow \mathbb{P}$. Then $I$-open maps are necessarily $I^{\prime}$-open. In particular, if $I$ and $I^{\prime}$ are naturally isomorphic, then an arrow is $I$-open iff it is $I^{\prime}$-open.

In the case of presheaves the definition of open map, translates via the Yoneda Lemma to an equivalent path-lifting property of $f$ :

Proposition 2.3 A morphism between presheaves is $\mathbb{P}$-open iff it is $\mathrm{y}_{\mathbb{P}}$-open.

In the main we shall work with open maps in presheaf categories; only rarely shall we need to make explicit which notion of openness is intended.

Open maps generalise functional bisimulations of process algebra (i.e., where the bisimulation relation is a function). A symmetric relation of bisimilarity is obtained through the presence of spans of surjective open maps. ${ }^{3}$ (Because presheaves may lack unique elements corresponding to initial states we insist on the surjectivity condition - otherwise any two presheaves would be related by a span of open maps from the empty presheaf.)

Definition 2.4 We say that presheaves $X, Y$ in $\widehat{\mathbb{P}}$ are $\mathbb{P}$-bisimilar iff there is a span of surjective open maps between them. This is equivalent to there being a subobject $R \hookrightarrow X \times Y$ such that the compositions with the projections

$$
R \hookrightarrow X \times Y \stackrel{\pi_{1}}{\rightarrow} X \text { and } R \hookrightarrow X \times Y \stackrel{\pi_{2}}{\rightarrow} Y
$$

are surjective open.

The following preservation property of open maps along adjunctions will be useful in Section 9 (see $[17,28]$ for other applications and a related result):

Lemma 2.5 If $\mathbb{P} \stackrel{H}{\longrightarrow} \mathcal{A} \underset{L}{\stackrel{R}{T}} \mathcal{B}$, are three functors with $L$ left adjoint to $R$, we have for every arrow $g$ in $\mathcal{B}$, that $R g$ is $H$-open iff $g$ is $L H$-open.

Proof: "only if": Suppose

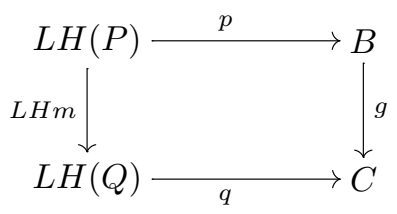

\footnotetext{
${ }^{3}$ Surjective maps in a presheaf category are those natural transformations between presheaves whose components are always surjective functions; surjective maps coincide with epimorphisms in presheaf categories.
} 
commutes. Then the following commutes as well:

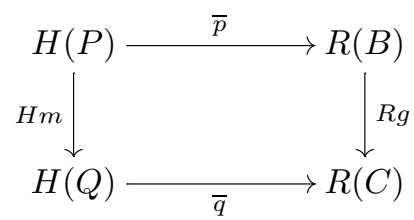

where $\bar{p}$ and $\bar{q}$ are the transpositions of $p$ and $q$ along the adjoint pair $L \dashv R$ [33]. Let, then $r: H(Q) \rightarrow R(B)$ be such that $r(H m)=\bar{p}$ and $(R g) r=\bar{q}$. Transposing $r$, gives $\bar{r}: L H(Q) \rightarrow B$ such that (see [33])

$$
\bar{r}(L H m)=\overline{(r(H m)}=\overline{\bar{p}}=p
$$

and

$$
g \bar{r}=\overline{(R g) r}=\overline{\bar{q}}=q .
$$

The "if" part uses the reverse argument, this time starting from a commuting square in $\mathcal{A}$.

In this paper the above proposition will often be applied in the context of presheaf categories; it then takes the form of the following lemma:

Lemma 2.6 If $I: \mathbb{P} \rightarrow \widehat{\mathbb{Q}}$ is a functor, then an arrow $h$ in $\widehat{\mathbb{Q}}$ is $I$-open iff $I^{*}(h)$ is $\mathrm{y}_{\mathbb{P}}$-open.

Proof: We have the following situation:

$$
\mathbb{P} \stackrel{\mathrm{y}_{\mathbb{P}}}{\longrightarrow} \widehat{\mathbb{P}} \underset{\operatorname{Lan}_{\mathrm{y}_{\mathbb{P}}}(I)}{\stackrel{I^{*}}{\leftrightarrows}} \widehat{\mathbb{Q}}
$$

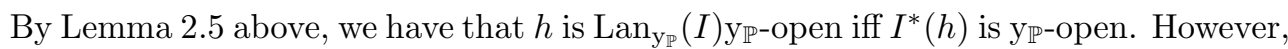
since $\mathrm{y}_{\mathbb{P}}$ is full and faithful, $\operatorname{Lan}_{\mathrm{y}}(I) \mathrm{y}_{\mathbb{P}} \cong I$, and so $I$-openness and $\operatorname{Lan}_{\mathrm{y}_{\mathbb{P}}}(I) \mathrm{y}_{\mathbb{P}^{-}}$ openness coincide.

We remark that categories of process models often fit the situation described in Lemma 2.5. For example $\mathcal{A}$ might be the category of labelled event structures, $\mathcal{B}$ the category of Petri nets, related by an adjunction with right adjoint $R$ "unfolding" a net to an event structure. Appropriate computation paths $\mathbb{P}$ are then finite labelled partial orders of events (pomsets) in event structures, with $H$ the inclusion of pomsets. The lemma then says that open maps, and so bisimulation, are preserved by the unfolding of nets. (See $[28,55]$ for more detail and further examples.)

\section{A result on open map preservation}

We are about to prove a key result, that colimit preserving functors, the mathematically natural maps between presheaf categories, preserve open maps and open map bisimulation. In preparation, it is helpful to think of a category of elements of a 
presheaf over $\mathbb{P}$ (see Definition A.13) as a transition system in the which the computation paths have shapes in $\mathbb{P}$. This point of view, in which the objects of the category of elements are regarded as states and its arrows as transitions, is emphasised in [56]. We examine how properties of maps between presheaves correspond to well-known properties of morphisms of transition systems [54].

Proposition 3.1 Let $f: X \rightarrow Y$ be a map in $\widehat{\mathbb{P}}$.

(i) Suppose $(\mathcal{E} l(f))(x)=y$ and $x \stackrel{e}{\longrightarrow} x^{\prime}$ in $\mathcal{E} l(X)$. Then, there is $y^{\prime}$ such that $(\mathcal{E l}(f))\left(x^{\prime}\right)=y^{\prime}$ and $y \stackrel{e}{\longrightarrow} y^{\prime}$ in $\mathcal{E} l(Y)$ :

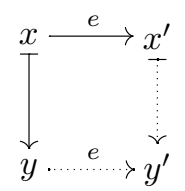

(ii) Suppose $(\mathcal{E} l(f))\left(x^{\prime}\right)=y^{\prime}$ and $y \stackrel{e}{\longrightarrow} y^{\prime}$ in $\mathcal{E l}(Y)$. Then, there is $x$ such that $(\mathcal{E} l(f))(x)=y$ and $x \stackrel{e}{\longrightarrow} x^{\prime}$ in $\mathcal{E} l(X)$ :

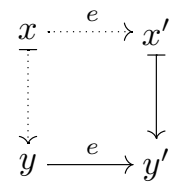

(iii) Assume $f$ is an open map. Then, $\mathcal{E} l(f)$ satisfies the condition that if $(\mathcal{E l}(f))(x)=$ $y$ and $y \stackrel{e}{\longrightarrow} y^{\prime}$ in $\mathcal{E} l(Y)$, then, there is $x^{\prime}$ such that $(\mathcal{E} l(f))\left(x^{\prime}\right)=y^{\prime}$ and $x \stackrel{e}{\longrightarrow} x^{\prime}$ in $\mathcal{E} l(X)$ :

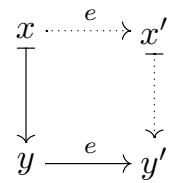

Conversely, if $\mathcal{E} l(f)$ satisfies this condition, then $f$ is an open map.

\section{Proof:}

(i) Directly from the functoriality of $\mathcal{E} l(f)$.

(ii) Directly from the naturality of $f$.

(iii) Directly from the quasi-pullback condition expressing the openness of $f$.

The property (iii) says that a map $f: X \rightarrow Y$ between presheaves is open exactly when $\mathcal{E} l(f): \mathcal{E} l(X) \rightarrow \mathcal{E} l(Y)$ is a "functional bisimulation" between categories of elements, viewed as transition systems (a functional bisimulation is a bisimulation $[37,42]$ whose graph is a function). From the point of view of transition systems, condition (ii) is expected when the transition systems are unfoldings (condition (ii) holds for instance in the categories of label-preserving morphisms of synchronisation trees and event structures $[54,28])$.

By combining properties (ii) and (iii) we immediately obtain that open maps reflect "zig-zags" in the following sense. 
Corollary 3.2 Assume $f$ is an open map. Suppose $(\mathcal{E} l(f))\left(x_{0}\right)=y_{0}$ and that

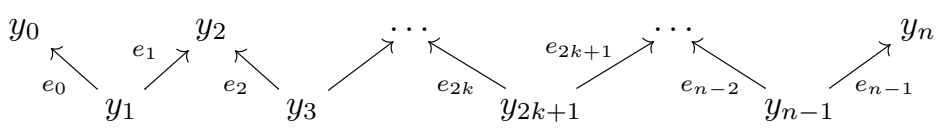

- a "zig-zag", in $\mathcal{E} l(Y)$. Then there is a corresponding "zig-zag"

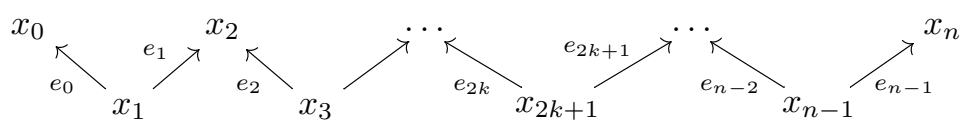

in $\mathcal{E l}(X)$ with $(\mathcal{E} l(f))\left(x_{i}\right)=y_{i}$ whenever $0 \leq i \leq n$.

Proof: We lift the $e_{n}$-arrows, when $n$ is even by (ii), and when $n$ is odd by (iii) of Proposition 3.1.

The next theorem, a major result of this paper, was first announced in [14].

Theorem 3.3 A colimit preserving functor between presheaf categories preserves open maps.

Proof: As $\widehat{\mathbb{P}}, \mathrm{y}_{\mathbb{P}}$ is a free colimit completion, to within isomorphism, any colimit preserving functor from $\widehat{\mathbb{P}}$ to $\widehat{\mathbb{Q}}$ can be obtained as a left Kan extension $\operatorname{Lan}_{\mathrm{y}} F$ of a functor $F: \mathbb{P} \rightarrow \widehat{\mathbb{Q}}$. Clearly if a functor preserves open maps, then so does any functor naturally isomorphic to it. So, without loss of generality, it suffices to show that, assuming a functor $F: \mathbb{P} \rightarrow \widehat{\mathbb{Q}}$, its left Kan extension $L=\operatorname{Lan}_{\mathrm{y}} F: \widehat{\mathbb{P}} \rightarrow \widehat{\mathbb{Q}}$ preserves open maps.

Let $Y$ be a presheaf in $\widehat{\mathbb{P}}$. Recall from the Appendix, A.4.2, that

$$
L(Y)=\operatorname{colim}\left(\mathcal{E} l(Y) \stackrel{\pi_{Y}}{\longrightarrow} \mathbb{P} \stackrel{F}{\longrightarrow} \widehat{\mathbb{Q}}\right) .
$$

Taking advantage of the concrete presentation of colimits in Set-see the Appendix, Proposition A.4, we can express $(L(Y))(Q)$, where $Q$ is an object of $\mathbb{Q}$, as a set of equivalence classes:

$$
(L(Y))(Q)=\sum_{(P, y) \in|\mathcal{E} l(Y)|}(F P)(Q) / \sim
$$

where $\sim$ is the least equivalence relation such that $((P, y), u) \sim\left(\left(P^{\prime}, y^{\prime}\right), u^{\prime}\right)$ if

$$
\exists e:(P, y) \rightarrow\left(P^{\prime}, y^{\prime}\right) \text { in } \mathcal{E} l(Y) . \quad(F e)_{Q}(u)=u^{\prime} .
$$

Thus, $((P, y), u) \sim\left(\left(P^{\prime}, y^{\prime}\right), u^{\prime}\right)$ iff there is a "zig-zag" in $\mathcal{E} l(Y)$, viz.

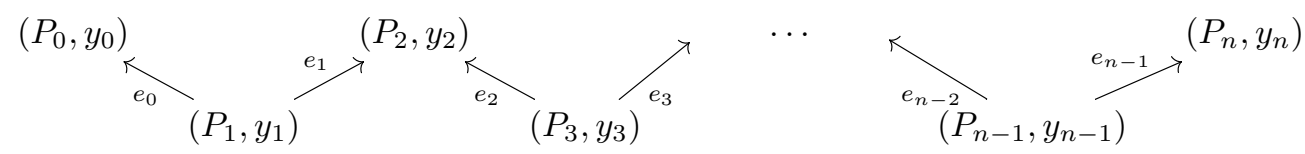

with

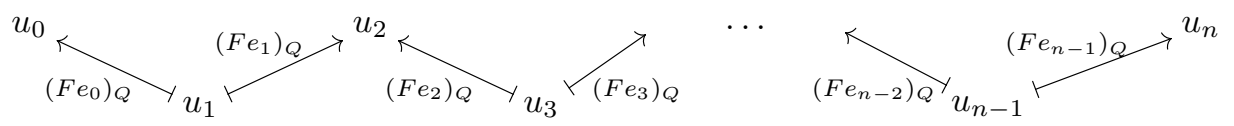


where $y=y_{0}, y^{\prime}=y_{n}$, and $u=u_{0}, u^{\prime}=u_{n}$.

For a presheaf $Y$ in $\widehat{\mathbb{P}}$, the components of the colimiting cone $\left\langle F P(Q) \stackrel{\gamma_{P, y}}{\longrightarrow}\right.$ $L Y(Q)\rangle_{(P, y) \in|\mathcal{E} l(Y)|}$ are given explicitly by

$$
\gamma_{P, y}(u)=\{((P, y), u)\}_{\sim} .
$$

It will be useful to understand the functorial actions of $L h$ and $L Y$ on representatives of $\sim$-equivalence classes.

For $m: Q \rightarrow Q^{\prime}$ in $\mathbb{Q}$,

$$
L Y(m)\left(\left\{\left(\left(P^{\prime}, y^{\prime}\right), w\right)\right\}_{\sim}\right)=\left\{\left(\left(P^{\prime}, y^{\prime}\right), F P^{\prime}(m)(w)\right)\right\}_{\sim} .
$$

The map $L Y(m)$ is the unique function, mediating between the colimiting cones $\left\langle F P(Q) \stackrel{\gamma_{P, y}}{\longrightarrow} L Y(Q)\right\rangle_{(P, y) \in|\mathcal{E} l(Y)|}$ and $\left\langle F P\left(Q^{\prime}\right) \stackrel{\gamma_{P, y}^{\prime}}{\longrightarrow} L Y\left(Q^{\prime}\right)\right\rangle_{(P, y) \in|\mathcal{E} l(Y)|}$, such that

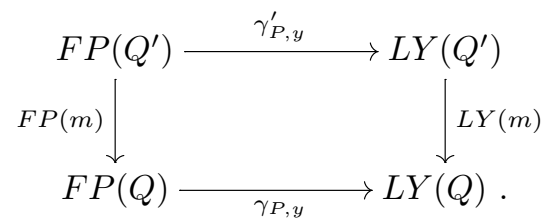

For $h: X \rightarrow Y$ in $\widehat{\mathbb{P}}$, the component of $L h$ at an object $Q$ is a function $(L h)_{Q}$ : $L X(Q) \rightarrow L Y(Q)$ such that

$$
(L h)_{Q}\left(\{((P, x), u)\}_{\sim}\right)=\left\{\left(\left(P, h_{P}(x)\right), u\right)\right\}_{\sim}
$$

- see the definition of $L h=\operatorname{Lan}_{\mathrm{yp}} F(h)$ in Appendix A.4.3.

Suppose now that $h: X \rightarrow Y$ is an open map in $\widehat{\mathbb{P}}$. In order to show that $L h$ is open we require that each naturality square

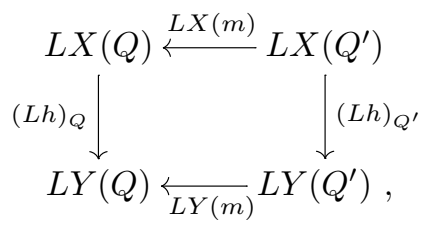

associated with $m: Q \rightarrow Q^{\prime}$, is a quasi-pullback.

To this end suppose that

$$
L Y(m)\left(\left\{\left(\left(P^{\prime}, y^{\prime}\right), w\right)\right\}_{\sim}\right)=(L h)_{Q}\left(\{((P, x), u)\}_{\sim}\right) .
$$

Then, from the action of $L Y(m)$ and $(L h)_{Q}$ on representatives, noted above,

$$
\left(\left(P, h_{P}(x)\right), u\right) \sim\left(\left(P^{\prime}, y^{\prime}\right), F P^{\prime}(m)(w)\right) .
$$

Hence $\left(\left(P, h_{P}(x)\right), u\right)$ and $\left(\left(P^{\prime}, y^{\prime}\right), F P^{\prime}(m)(w)\right)$ are connected via a "zig-zag" in 
$\mathcal{E} l(Y)$, viz.

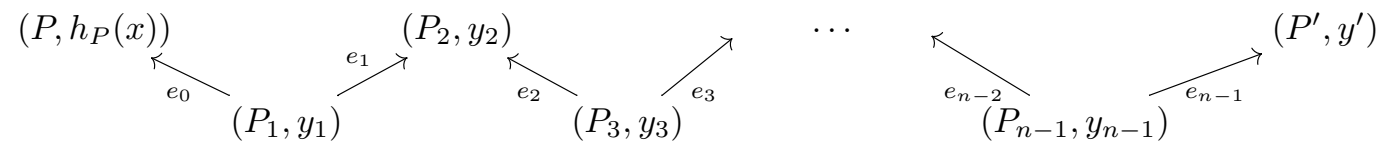

with

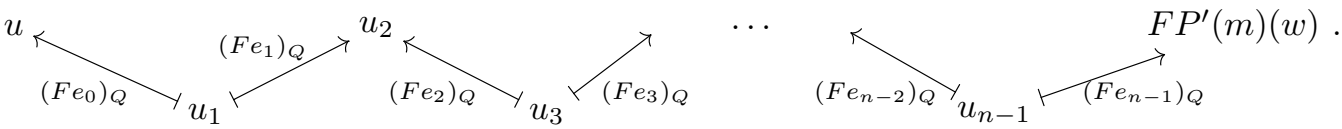

But, by Corollary 3.2, this "zig-zag" is reflected by a "zig-zag" in $\mathcal{E l}(X)$, viz.<smiles>C=[Te]</smiles>

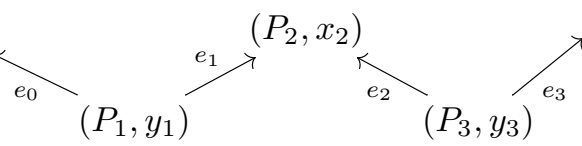

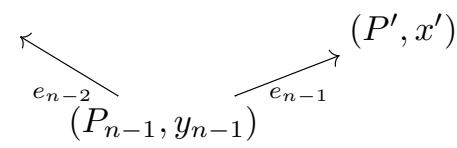

where still
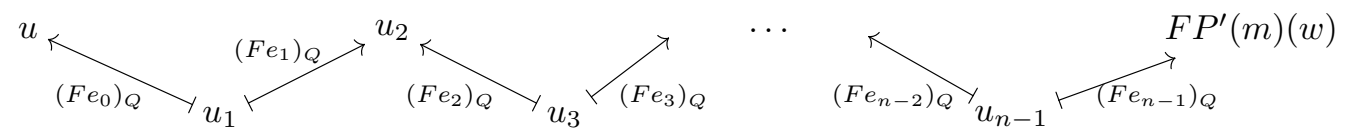

with $h_{P^{\prime}}\left(x^{\prime}\right)=y^{\prime}$. Thus,

$$
((P, x), u) \sim\left(\left(P^{\prime}, x^{\prime}\right), F P^{\prime}(m)(w)\right) .
$$

Recalling the action of $L X(m)$ and $(L h)_{Q^{\prime}}$ on representatives,

$$
L X(m)\left(\left\{\left(\left(P^{\prime}, x^{\prime}\right), w\right)\right\}_{\sim}\right)=\left\{\left(\left(P^{\prime}, x^{\prime}\right), F P^{\prime}(m)(w)\right)\right\}_{\sim}=\{((P, x), u)\}_{\sim},
$$

and

$$
(L h)_{Q^{\prime}}\left(\left\{\left(\left(P^{\prime}, x^{\prime}\right), w\right)\right\}_{\sim}\right)=\left\{\left(\left(P^{\prime}, h_{P^{\prime}}\left(x^{\prime}\right)\right), w\right)\right\}_{\sim}=\left\{\left(\left(P^{\prime}, y^{\prime}\right), w\right)\right\}_{\sim} .
$$

Hence we fulfil the quasi-pullback condition, thus ensuring that $L h: L X \rightarrow L Y$ is open in $\widehat{\mathbb{Q}}$.

Colimit preserving functors between presheaf categories preserve open map bisimulation.

Corollary 3.4 If presheaves $X$ and $Y$ are $\mathbb{P}$-bisimilar and $F: \widehat{\mathbb{P}} \rightarrow \widehat{\mathbb{Q}}$ is a colimit preserving functor, then $F(X)$ is $\mathbb{Q}$-bisimilar to $F(Y)$.

Proof: If $X \stackrel{f}{\longleftarrow} Z \stackrel{g}{\longrightarrow} Y$ is a span of $\mathbb{P}$-open maps then, by Corollary 3.3,

$$
F(X) \stackrel{F(f)}{\longleftarrow} F(Z) \stackrel{F(g)}{\longrightarrow} F(Y)
$$


is a span of $\mathbb{Q}$-open maps. Moreover if $f$ and $g$ are surjective, so are $F(f)$ and $F(g)$. In fact in any category an arrow $e: C \rightarrow D$ is an epimorphism iff the following diagram is a pushout

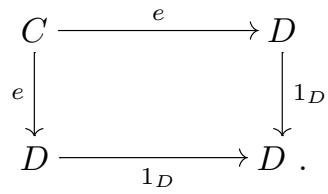

Since $F$ preserves colimits, in particular it preserves pushouts.

Theorem 3.3 and Corollary 3.4 have many applications. For now, recall from Appendix A.4.6, that a functor $F: \mathbb{P} \rightarrow \mathbb{Q}$, between small categories $\mathbb{P}$ and $\mathbb{Q}$, induces a triple of adjoints

$$
F_{!} \dashv F^{*} \dashv F_{*}: \widehat{\mathbb{P}} \rightarrow \widehat{\mathbb{Q}}
$$

Both $F_{!}$and $F^{*}$ are colimit preserving as they are left adjoints. Hence, $F$ ! sends $\mathbb{P}$-open maps to $\mathbb{Q}$-open maps, and so bisimilar presheaves in $\widehat{\mathbb{P}}$ to bisimilar presheaves in $\widehat{\mathbb{Q}}$. In the other direction, $F^{*}$ sends $\mathbb{Q}$-open maps to $\mathbb{P}$-open maps, and bisimilar presheaves in $\widehat{\mathbb{Q}}$ to bisimilar presheaves in $\widehat{\mathbb{P}}$. We might, for example, take $\mathbb{P}$ to be the partial order category of non-empty strings over some alphabet $L$ and $\mathbb{Q}$ to be the category of nonempty, finite pomsets with labels in $L$. See [28] for the detailed description of these categories, and the explanation of the presheaf categories $\widehat{\mathbb{P}}$ as synchronisation trees with $\mathbb{P}$-bisimulation being strong bisimulation, and $\widehat{\mathbb{Q}}$ as including event structures with labels in $L$, with $\mathbb{Q}$-bisimulation being hereditary history preserving bisimulation. There is an obvious inclusion of strings into pomsets giving rise to a functor $F: \mathbb{P} \rightarrow \mathbb{Q}$. In this case, $F$ is the inclusion of synchronisation trees in event structures, and its right adjoint $F^{*}$ the operation which serialises an event structure to produce a tree. That, for example $F^{*}$ preserves open map bisimulation implies that two hereditary history preserving bisimilar event structures are sent to strongly bisimilar synchronisation trees. The papers $[14,15]$ contain several examples directly using this result, including a characterisation of a well known refinement operation on event structures [19] as an instance of $F_{1}$.

\section{The bicategory Prof and the 2-category Cocont}

Presheaf categories are free colimit completions. Morphisms between them are naturally taken to be colimit preserving functors. In order to study the relation between presheaf categories we consider the following 2-category:

Definition 4.1 Define Cocont to consist of

- objects: small categories, $\mathbb{P}, \mathbb{Q}, \mathbb{R}, \ldots$

- arrows: colimit preserving functors between the corresponding presheaf categories, i.e., $F$ is an arrow from $\mathbb{P}$ to $\mathbb{Q}$, if it is a colimit preserving functor $F: \widehat{\mathbb{P}} \rightarrow \widehat{\mathbb{Q}}$

- 2-cells: natural transformations between such functors. 
The composition of arrows is the usual composition of functors. The vertical and horizontal composition of 2-cells are those of natural transformations [33].

As we saw, to within isomorphism, colimit preserving functors $\widehat{\mathbb{P}} \rightarrow \widehat{\mathbb{Q}}$ correspond to functors $\mathbb{P} \rightarrow \widehat{\mathbb{Q}}$, which correspond by "uncurrying" to functors $\mathbb{P} \times \mathbb{Q}^{\text {op }} \rightarrow$ Set. Functors of this latter kind are often called profunctors (or bimodules or distributors $)[6,31,5]$. For a functor $F: \mathbb{P} \times \mathbb{Q}^{\text {op }} \rightarrow$ Set, we write $F: \mathbb{P} \rightarrow \rightarrow \mathbb{Q}$ to signify that $F$ is a profunctor from $\mathbb{P}$ to $\mathbb{Q}$. Often operations are best described on profunctors, which provide an alternative (bicategorical) presentation of Cocont.

Definition 4.2 The bicategory Prof of profunctors is defined to consist of

- objects: small categories, $\mathbb{P}, \mathbb{Q}, \mathbb{R} \ldots$

- arrows: Profunctors $F: \mathbb{P} \longrightarrow \mathbb{Q}$

- 2-cells: $\alpha: F \Rightarrow G$, natural transformations between profunctors.

The vertical composition of 2-cells is the usual (vertical) composition of natural transformations. Horizontal composition of both arrows and 2-cells is described in terms of coend formulae. Given two arrows $\mathbb{P} \stackrel{F}{\rightarrow} \mathbb{Q} \stackrel{G}{\rightarrow} \mathbb{R}$, consider the following functor,

$$
\mathbb{P} \times \mathbb{Q}^{\text {op }} \times \mathbb{Q} \times \mathbb{R}^{\text {op }} \stackrel{F \times G}{\longrightarrow} \text { Set } \times \text { Set } \stackrel{\times}{\longrightarrow} \text { Set }
$$

that to each 4-tuple of objects $P, Q, Q^{\prime}, R$ associates the set $F(P, Q) \times G\left(Q^{\prime}, R\right)$, with the obvious actions on morphisms derived from those of $F$ and $G$. Using coends (see Appendix A), one defines the composition of $F$ and $G$ as arrows of Prof as

$$
G F(P, R)=\int^{Q} F(P, Q) \times G(Q, R)
$$

and for any $f: P \rightarrow P^{\prime}$ and $g: R^{\prime} \rightarrow R$, defines

$$
G F(f, g)=\int^{Q} F(f, Q) \times G(Q, g): G F(P, R) \rightarrow G F\left(P^{\prime}, R^{\prime}\right) .
$$

To specify the horizontal composition of 2-cells, suppose we have the following situation

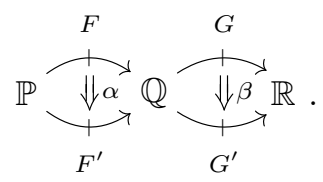

Define $\beta \alpha: G F \Rightarrow G^{\prime} F^{\prime}$, the horizontal composition of the two cells $\alpha$ and $\beta$, to be the natural transformation with components

$$
(\beta \alpha)_{\langle P, R\rangle}=\int^{Q} \alpha_{\langle P, Q\rangle} \times \beta_{\langle Q, R\rangle}
$$

As for identities, these are just the hom-functors. Given any small category $\mathbb{P}$ define

$$
1_{\mathbb{P}}: \mathbb{P} \times \mathbb{P}^{\text {op }} \rightarrow \text { Set } \quad \text { so that } \quad\left(P, P^{\prime}\right) \mapsto \mathbb{P}\left(P^{\prime}, P\right) \text {. }
$$


Obviously "currying" $1_{\mathbb{P}}$ yields the Yoneda embedding $\mathrm{y}_{\mathbb{P}}$. The associativity morphisms and those for left and right identities are derived from the universal property that defines coends.

Profunctors subsume presheaves:

Proposition 4.3 A presheaf category, $\widehat{\mathbb{P}}$, is isomorphic to the category $\operatorname{Prof}(\mathbb{1}, \mathbb{P})$ of profunctors from the terminal category to the category $\mathbb{P}$. The terminal category $\mathbb{1}$ consists of one object $*$ and its identity arrow $1_{*}$. Under the isomorphism, a presheaf $X$ in $\widehat{\mathbb{P}}$ corresponds to a profunctor $X^{\prime}$ where $X^{\prime}(*, P)=X(P)$ and $X^{\prime}\left(1_{*}, f\right)=X(f)$ for any arrow $f: P \rightarrow Q$ in $\mathbb{P}$. A natural transformation $\alpha$ between presheaves corresponds to a 2 -cell $\alpha^{\prime}$ where $\alpha_{\langle *, P\rangle}^{\prime}=\alpha_{P}$.

Notation: It is often useful to identify profunctors with functors $\mathbb{P} \rightarrow \widehat{\mathbb{Q}}$ (after "currying") via the isomorphism

$$
\left[\mathbb{P} \times \mathbb{Q}^{\text {op }}, \text { Set }\right] \cong\left[\mathbb{P},\left[\mathbb{Q}^{\text {op }}, \text { Set }\right]\right]
$$

between functor categories. Profunctors $F: \mathbb{P} \rightarrow \mathbb{Q}$ correspond to functors $\bar{F}: \mathbb{P} \rightarrow$ $\widehat{\mathbb{Q}}$, by "currying", where $\bar{F}(P)(Q)=F(P, Q)$. We will use the same notation for the inverse "uncurrying" operation; for a functor $G: \mathbb{P} \rightarrow \widehat{\mathbb{Q}}$ we will write $\bar{G}: \mathbb{P} \rightarrow \mathbb{Q}$ for the corresponding profunctor. The same notation will be used for the action of the isomorphism on natural transformations between such functors; when $\alpha: F \Rightarrow F^{\prime}$ between profunctors we write $\bar{\alpha}: \bar{F} \Rightarrow \bar{F}^{\prime}$ for the corresponding natural transformation between their curried forms, and vice versa.

The composition of profunctors $F: \mathbb{P} \rightarrow \mathbb{Q}$ and $G: \mathbb{Q} \rightarrow \mathbb{R}$ can be expressed in terms of left Kan extensions. Using a choice of left Kan extension,

$$
\overline{G F} \cong \operatorname{Lan}_{\mathrm{y}_{Q}}(\bar{G}) \circ \bar{F},
$$

where the second composition is the usual composition of functors. In fact, since colimits in presheaf categories are computed pointwise, we have from Appendix A.4.2 that for any object $P$ of $\mathbb{P}$ and object $R$ of $\mathbb{R}$,

$$
\begin{aligned}
\overline{\left(\operatorname{Lan}_{\mathrm{y}_{Q}}(\bar{G}) \circ \bar{F}\right)}(P, R) & =\left(\left(\operatorname{Lan}_{\mathrm{y}_{Q}}(\bar{G}) \circ \bar{F}\right)(P)\right)(R) \\
& \cong\left(\int^{Q} \bar{F}(P)(Q) \cdot \bar{G}(Q)\right)(R) \\
& =\int^{Q} \bar{F}(P)(Q) \times \bar{G}(Q)(R) \\
& =\int^{Q} F(P, Q) \times G(Q, R) .
\end{aligned}
$$

Prof and Cocont are equivalent as bicategories. In defining the biequivalence $\Lambda$ from Prof to Cocont, we assume for every profunctor $F: \mathbb{P} \rightarrow \mathbb{Q}$ a choice $\left(\operatorname{Lan}_{\mathrm{y}}(\bar{F}), \theta^{F}\right)$ of left Kan extension; we will write $F^{\dagger}$ for $\operatorname{Lan}_{\mathrm{y}}(\bar{F})$. Define $\Lambda_{(\mathbb{P}, \mathbb{Q})}$ : $\operatorname{Prof}(\mathbb{P}, \mathbb{Q}) \rightarrow \operatorname{Cocont}(\mathbb{P}, \mathbb{Q})$ to be the functor which maps $F$ to $F^{\dagger}$ and $\alpha: F \Rightarrow G$ to the unique $\alpha^{\dagger}$ such that $\left(\alpha^{\dagger} \mathrm{y}_{\mathbb{P}}\right) \cdot \theta^{F}=\theta^{G} \cdot \bar{\alpha}$, given by the universal property of Kan extensions. Notice that $\Lambda$ is the identity on objects. Since $\left(1_{\widehat{\mathbb{p}}}, 1_{\mathrm{y \mathbb {P }}}\right)$ is a left 
Kan extension of $\mathrm{y}_{\mathbb{P}}$ along itself, we can further assume that $\Lambda_{(\mathbb{P}, \mathbb{Q})}\left(1_{\mathbb{P}}\right)=1_{\widehat{\mathbb{P}}}$. In the converse direction, from Cocont to Prof, define $\Xi_{(\mathbb{P}, \mathbb{Q})}$ simply by precomposing with $\mathrm{y}_{\mathbb{P}}$, followed by "uncurrying". We have the following:

Proposition $4.4 \Lambda$ and $\Xi$ are bicategorical homomorphisms [48]

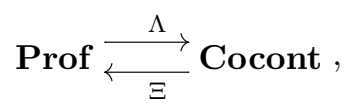

which are the identity on objects, send identity arrows to identity arrows and are such that for any two small categories $\mathbb{P}, \mathbb{Q}$, the functors $\Lambda_{(\mathbb{P}, \mathbb{Q})}$ and $\Xi_{(\mathbb{P}, \mathbb{Q})}$ are equivalences of categories, pseudo inverses to each other.

With the view of Prof and Cocont as "categories" of domains of nondeterministic processes, the techniques required to solve recursive domain equations are explored in $[12]$.

\section{The structure of Prof}

It has been remarked, for example in [30], that Prof has enough structure to be, what might be called, a compact closed bicategory. To see this, we first need to define certain bicategorical limits explicitly.

\subsection{Pseudo-products and -coproducts}

Definition 5.1 (Pseudo-products and -coproducts) In a bicategory $\mathcal{B}$, a pseudoproduct of two objects $B, C$, is given by an object $D$ and an equivalence of categories

$$
\mathcal{B}(E, B) \times \mathcal{B}(E, C) \simeq \mathcal{B}(E, D)
$$

pseudo-natural in E; more explicitly a pseudo-product is given by a span of arrows

$$
B \stackrel{\pi_{1}}{\longleftarrow} D \stackrel{\pi_{2}}{\longrightarrow} C
$$

such that:

1. For any other span, $B \stackrel{f}{\longleftarrow} E \stackrel{g}{\longrightarrow} C$, there exists an $h: E \rightarrow D$ and isomorphic

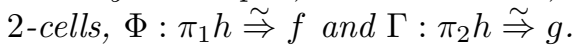

2. For any two arrows $h, k: E \rightarrow D$ and 2 -cells, $\sigma_{i}: \pi_{i} h \Rightarrow \pi_{i} k$, for $i=1,2$, there exists a unique $\sigma: h \Rightarrow k$, such that $\sigma_{i}=\pi_{i} \sigma$.

If the equivalences are isomorphisms, we shall say that the product is strict.

One defines pseudo-coproducts in a dual fashion.

Remark: Observe that our terminology for bicategorical limits clashes with that often employed in the literature, e.g., in [48], where one would talk about "bilimits" rather than "pseudo-limits" which there denote a stricter notion. We follow the practice of [16]. 
Prof has strict pseudo products $(\&)$ and coproducts $(\oplus)$ and they coincide on objects. Let $\mathbb{P}$ and $\mathbb{Q}$ be two small categories, define

$$
\mathbb{P} \& \mathbb{Q} \stackrel{\text { def }}{=} \mathbb{P}+\mathbb{Q} \stackrel{\text { def }}{=} \mathbb{P} \oplus \mathbb{Q},
$$

where $\mathbb{P}+\mathbb{Q}$ is the usual disjoint union of small categories with inclusions $i n_{\mathbb{P}}$ and $i n_{\mathbb{Q}}$. Further define $\pi_{\mathbb{P}}: \mathbb{P} \& \mathbb{Q} \longrightarrow \mathbb{P}$ by $\pi_{\mathbb{P}}\left(i n_{\mathbb{P}}(P), P^{\prime}\right)=\mathbb{P}\left(P^{\prime}, P\right)$ and $\pi_{\mathbb{P}}\left(i n_{\mathbb{Q}}(Q), P^{\prime}\right)=\emptyset$ and symmetrically $\pi_{\mathbb{Q}}$. The profunctor $i_{\mathbb{P}}: \mathbb{P}-r \rightarrow \mathbb{P} \oplus \mathbb{Q}$ is defined as the uncurrying of $\mathrm{y}_{\mathbb{P}+\mathbb{Q}} i n_{\mathbb{P}}$.

Notice that $\widehat{\mathbb{P} \& \mathbb{Q}}$ is isomorphic to $\widehat{\mathbb{P}} \times \widehat{\mathbb{Q}}$; a presheaf $Z$ in $\widehat{\mathbb{P}+\mathbb{Q}}$ restricts to presheaf $X$ over $\mathbb{P}$ and one $Y$ over $\mathbb{Q}$, and so splits into a pair $(X, Y)$. (We will often present a presheaf in $\widehat{\mathbb{P} \& \mathbb{Q}}$ as a pair $(X, Y)$.) This accounts for the strictness of product and coproduct.

Definition 5.2 (Pseudo-initial, -terminal and -zero object) In a bicategory $\mathcal{B}$ a pseudo-initial object 0 is an object such that $\mathcal{B}(0, B) \simeq \mathbb{1}$ for every object $B$ of $\mathcal{B}$. (The terminal category $\mathbb{1}$ consists of a single object with a single morphism, the identity.)

Dually one defines what a pseudo-terminal object is.

An object is a pseudo-zero object, if it is both pseudo-initial and-terminal.

If the equivalences are isomorphisms one talks of strict pseudo-initial, -terminal and -zero objects.

Prof has a (strict) pseudo-zero object. Take the initial category, $\mathbb{O}$, with no objects and no arrows. Of course the zero object is the unit for the product/coproduct bifunctor.

\subsection{Tensor}

We define a tensor $\otimes:$ Prof $\times$ Prof $\rightarrow$ Prof in Prof as follows:

- On objects: $\mathbb{P} \otimes \mathbb{Q} \stackrel{\text { def }}{=} \mathbb{P} \times \mathbb{Q}$, the product of categories

- On arrows: If $F: \mathbb{P}-1 \rightarrow \mathbb{P}^{\prime}$ and $G: \mathbb{Q}-1 \rightarrow \mathbb{Q}^{\prime}$,

$$
\begin{aligned}
F \otimes G: \mathbb{P} \times \mathbb{Q} \times \mathbb{P}^{\prime \text { op }} \times \mathbb{Q}^{\prime \text { op }} & \rightarrow \text { Set } \\
\left(P, Q, P^{\prime}, Q^{\prime}\right) & \mapsto F\left(P, P^{\prime}\right) \times G\left(Q, Q^{\prime}\right)
\end{aligned}
$$

- On 2-cells: if $\alpha: F \Rightarrow F^{\prime}$ and $\beta: G \Rightarrow G^{\prime}$, then

$$
(\alpha \otimes \beta)_{\left(P, Q, P^{\prime}, Q^{\prime}\right)}=\alpha_{\left(P, P^{\prime}\right)} \times \beta_{\left(Q, Q^{\prime}\right)} .
$$

The terminal category $\mathbb{1}$ is a neutral element for $\otimes$.

Tensor classifies "bilinear" maps. For small categories $\mathbb{P}, \mathbb{Q}, \mathbb{R}$, a functor $G$ : $\widehat{\mathbb{P} \& \mathbb{Q}} \rightarrow \widehat{\mathbb{R}}$ is bilinear if it is "linear" in each argument, i.e. $G(-, Y)$ and $G(X,-)$ are colimit preserving for any $X \in \widehat{\mathbb{P}}$ and $Y \in \widehat{\mathbb{Q}}$. Let $\operatorname{Bilin}(\mathbb{P} \& \mathbb{Q}, \mathbb{R})$ be the category of bilinear functors from $\widehat{\mathbb{P \& Q}}$ to $\widehat{\mathbb{R}}$, related by natural transformations. 
Proposition 5.3 There is an equivalence of categories

$$
\operatorname{Prof}(\mathbb{P} \otimes \mathbb{Q}, \mathbb{R}) \simeq \operatorname{Bilin}(\mathbb{P} \& \mathbb{Q}, \mathbb{R}) .
$$

The equivalence is given by composition with a functor $J^{*}$, obtained in the following way.

Let $J: \mathbb{P} \otimes \mathbb{Q} \rightarrow \widehat{\mathbb{P \& Q}}$ be the full and faithful functor taking $(P, Q)$ to the pair of presheaves $\left(\mathrm{y}_{\mathbb{P}} P, \mathrm{y}_{\mathbb{Q}} Q\right)$. For profunctors $F: \mathbb{P} \otimes \mathbb{Q} \rightarrow \rightarrow \mathbb{R}$, consider their left Kan extensions along $J$ :

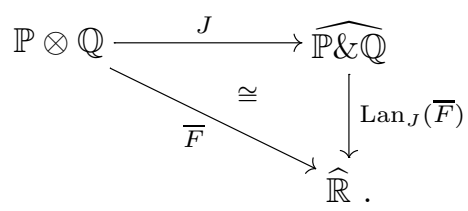

Note that by Proposition A.14 in the Appendix, we can factor the left Kan extension as

$$
\operatorname{Lan}_{J}(\bar{F}) \cong \operatorname{Lan}_{\mathrm{y}_{\mathbb{P} \otimes \mathbb{Q}}}(\bar{F}) \circ J^{*},
$$

where $J^{*}: \widehat{\mathbb{P \& Q}} \rightarrow \widehat{\mathbb{P} \otimes \mathbb{Q}}$ is given by

$$
\left(J^{*}(X, Y)\right)(P, Q)=\widehat{\mathbb{P \& Q}}(J(P, Q),(X, Y)) \cong X(P) \times Y(Q) .
$$

Because product in Set preserves colimits in each argument separately, it is easy to see that any functor $\operatorname{Lan}_{J}(\bar{F})$ is bilinear. Moreover, as presheaves are colimits of representables, any bilinear functor $G$ is determined by its restriction $G \circ J$ and so can be obtained up to isomorphism as such a left Kan extension. The equivalence between $\operatorname{Prof}(\mathbb{P} \otimes \mathbb{Q}, \mathbb{R})$ and $\operatorname{Bilin}(\mathbb{P} \& \mathbb{Q}, \mathbb{R})$ now follows by Proposition A.12 in the Appendix.

\subsection{Dualiser}

We define a dualiser in Prof. We write Prof ${ }^{\text {op }}$ for the opposite bicategory which reverses the direction of the 1-cells but not that of the 2-cells in Prof. Define the dualiser, $(-)^{\perp}$ : Prof $\rightarrow$ Prof $^{\text {op }}$, as follows:

- On objects: $\mathbb{P}^{\perp}=\mathbb{P}^{o p}$.

- On arrows: Given $F: \mathbb{P} \mapsto \mathbb{Q}$, define $F^{\perp}: \mathbb{Q}^{\perp} \longrightarrow \mathbb{P}^{\perp}$ as $F^{\perp}(Q, P)=F(P, Q)$.

- On 2-cells: If $\alpha: F \Rightarrow F^{\prime}$, then $\alpha^{\perp}: F^{\perp} \Rightarrow F^{\prime} \perp$, with $\alpha_{\langle Q, P\rangle}^{\perp}=\alpha_{\langle P, Q\rangle}$.

This definition of dualiser is straightforward and direct in contrast to the definition of the corresponding pseudo-functor on Cocont. The bicategory Prof might reasonably be called a $*$-autonomous bicategory [4].

\subsection{Function space}

Combining tensor and dualiser, yields a "linear function space". Define the pseudo functor $\multimap$ : Prof ${ }^{\circ \mathrm{p}} \times$ Prof $\rightarrow$ Prof as $\multimap=\otimes \circ\left((-)^{\perp} \times 1\right)$, so $\mathbb{P} \multimap \mathbb{Q}=\mathbb{P}^{\mathrm{op}} \times \mathbb{Q}$, for any small categories $\mathbb{P}$ and $\mathbb{Q}$. 
There is the following chain of natural isomorphisms for any small categories, $\mathbb{P}, \mathbb{Q}, \mathbb{R}:$

$$
\begin{aligned}
\operatorname{Prof}(\mathbb{P} \otimes \mathbb{Q}, \mathbb{R}) & \stackrel{\text { def }}{=} \operatorname{CAT}\left(\mathbb{P} \times \mathbb{Q} \times \mathbb{R}^{\text {op }}, \text { Set }\right) \\
& \cong \operatorname{CAT}\left(\mathbb{P}, \widehat{Q^{\text {op }} \times \mathbb{R}}\right) \\
& \stackrel{\text { def }}{=} \operatorname{CAT}\left(\mathbb{P}, \widehat{\mathbb{Q}^{\perp} \otimes \mathbb{R}}\right) \\
& \cong \operatorname{CAT}\left(\mathbb{P} \times\left(\mathbb{Q}^{\perp} \otimes \mathbb{R}\right)^{\text {op }}, \text { Set }\right) \\
& \stackrel{\text { def }}{=} \operatorname{Prof}\left(\mathbb{P}, \mathbb{Q}^{\perp} \otimes \mathbb{R}\right) \\
& \stackrel{\text { def }}{=} \operatorname{Prof}(\mathbb{P}, \mathbb{Q} \multimap \mathbb{R}) .
\end{aligned}
$$

The resultant isomorphism

$$
\operatorname{Prof}(\mathbb{P} \otimes \mathbb{Q}, \mathbb{R}) \cong \operatorname{Prof}(\mathbb{P}, \mathbb{Q} \multimap \mathbb{R})
$$

simply sets up a correspondence between profunctors $H:(\mathbb{P} \times \mathbb{Q}) \times \mathbb{R}^{\text {op }} \rightarrow$ Set on the left and profunctors $\bar{H}: \mathbb{P} \times\left(\mathbb{Q}^{\text {op }} \times \mathbb{R}\right)^{\text {op }} \rightarrow$ Set on the right, where $\bar{H}(P,(Q, R))=$ $H((P, Q), R)$. The isomorphism is pseudo-natural (or a strong transformation) in $\mathbb{P}$ and $\mathbb{Q}$ making a pseudo-adjunction (or biadjunction) between two copies of Prof $[48$, 45]:

Proposition 5.4 For any small category $\mathbb{Q}$, the pseudo-functor $-\otimes \mathbb{Q}$ is a left pseudoadjoint to $\mathbb{Q} \multimap-$.

\subsection{Linear logic}

We might summarise, informally and imprecisely, by saying that Prof is a compact closed bicategory.

From a logical point of view, Prof forms an interpretation of classical linear logic [18] once it is equipped with a suitable exponential, and so provides a basis for a rich linear type discipline. Though, as a model of classical linear logic, Prof is somewhat degenerate; the operations 28 ("par") and $\otimes$ ("tensor") coincide as do \& ("product") and $\oplus$ ("sum").

Looking ahead, the pseudo-comonad ! of Section 7, freely adjoining finite colimits, can play the role of the linear logic exponential. Its co-Kleisli bicategory in which the arrows of Prof are expanded to profunctors of the kind $! \mathbb{P} \rightarrow \mathbb{Q}$ is equivalent (as bicategories) to the 2-category of filtered colimit preserving functors between presheaf categories. This 2-category is cartesian closed with function spaces constructed as $! \mathbb{P} \multimap \mathbb{Q}$, for small categories $\mathbb{P}, \mathbb{Q}$; the key fact here is that ! satisfies the Seely condition [47] that there is an isomorphism of categories

$$
!(\mathbb{P} \& \mathbb{Q}) \cong ! \mathbb{P} \otimes ! \mathbb{Q}
$$

a presheaf over $\mathbb{P} \& \mathbb{Q}$ which is a finite colimit of representables splits into a pair of presheaves one over $\mathbb{P}$ and one over $\mathbb{Q}$, each of which is a finite colimit of representables.

Other candidates for exponentials are presented in Section 9. 


\section{Open map bisimulation in Prof}

For any categories $\mathbb{P}, \mathbb{Q}$, the category $\operatorname{Prof}(\mathbb{P}, \mathbb{Q})$ is identical with the presheaf category $\widehat{\mathbb{P o p} \times \mathbb{Q}}$; the 2 -cells in Prof are identical with arrows between presheaves. We inherit from presheaf categories a definition of open 2-cells in Prof. We will show that the horizontal composition in Prof of open 2-cells gives an open 2-cell, and consequently that horizontal composition preserves bisimulation. We saw a special case of this in Section 3, where we showed that colimit preserving functors between presheaf categories preserve open maps, and so open map bisimulation.

Definition 6.1 Let $\alpha: F \Rightarrow F^{\prime}$, be a 2-cell between two profunctors $F, F^{\prime}: \mathbb{P} \rightarrow \mathbb{Q}$. Define $\alpha$ to be open if it is open as an arrow of $\widehat{\mathbb{P o p} \times \mathbb{Q}}$

We unpack this definition. Since $\alpha$ is regarded as a natural transformation between two presheaves, its being open amounts to it satisfying the quasi-pullback condition of Definition 2.1. Suppose, that $\left\langle f^{\circ \mathrm{op}}, g\right\rangle:\langle P, Q\rangle \rightarrow\left\langle P^{\prime}, Q^{\prime}\right\rangle$ is an arrow in $\mathbb{P}^{\mathrm{op}} \times \mathbb{Q}$, then the following square must be a quasi-pullback in Set:

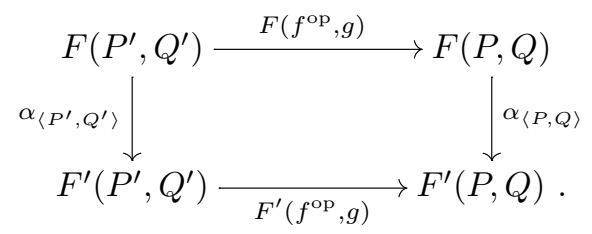

If we instantiate one of the two arguments $f$ or $g$ to be the identity arrow, on $P$ and $Q$, respectively, this immediately implies that the corresponding natural transformations,

$$
\begin{aligned}
& \bar{\alpha}_{P}: F(P,-) \Rightarrow F^{\prime}(P,-) \text { and } \\
& \bar{\alpha}_{Q}^{\perp}: F(-, Q) \Rightarrow F^{\prime}(-, Q)
\end{aligned}
$$

are $\mathbb{Q}$-open and $\mathbb{P}^{\text {op }}$-open respectively. The converse holds too:

Proposition 6.2 Let $\alpha: F \Rightarrow F^{\prime}$ be a natural transformation between two presheaves $F, F^{\prime} \in \widehat{\mathbb{P o p} \times \mathbb{Q}}$, then $\alpha$ is $(\mathbb{P}$ op $\times \mathbb{Q})$-open iff for any object $P$ of $\mathbb{P}$ and $Q$ of $\mathbb{Q}$, the corresponding natural transformations $\bar{\alpha}_{P}$ and $\bar{\alpha}_{Q}^{\perp}$ are $\mathbb{Q}$-open and $\mathbb{P}^{\mathrm{op}}$-open, respectively.

Proof: The discussion above shows "only if". For the converse, observe that, via the functoriality of $F$, the diagram (1) above can be rewritten as:

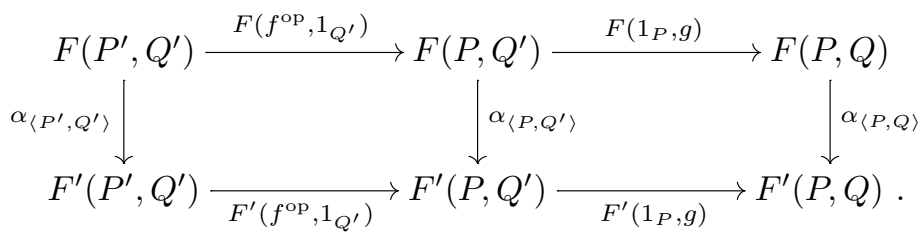

It is easy now to verify that the composition of the two quasi-pullback squares is a quasi-pullback square.

Consequently: 
Proposition 6.3 Let $\alpha: F \Rightarrow F^{\prime}$ be a 2-cell in Prof. The 2-cell $\alpha$ is open in Prof iff the 2 -cell $\alpha^{\perp}$ is open.

Proof: From Proposition 6.2 by dualising.

Proposition 6.4 According to the isomorphism between a presheaf category $\widehat{\mathbb{Q}}$ and the hom-category $\operatorname{Prof}(\mathbb{1}, \mathbb{Q})$ (cf.Proposition 4.3), a natural transformation between presheaves is open iff it is open as a 2-cell between the corresponding profunctors.

Proof: By specialising Proposition 6.2 to the case when $\mathbb{P}$ is $\mathbb{1}$.

Since open maps compose, and epimorphisms obviously compose, it is clear that the vertical composition of two (surjective) open 2-cells is a (surjective) open 2-cell. Our next goal is to show that the horizontal composition of 2-cells preserves (surjective) open maps, and so bisimulation.

\section{Theorem 6.5 (i) If}

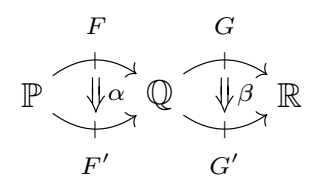

are two consecutive open 2-cells of Prof, then their horizontal composition $\beta \alpha$ is an open 2 -cell.

(ii) Suppose profunctors $F, F^{\prime}: \mathbb{P} \longrightarrow \mathbb{Q}$ are open map bisimilar and that profunctors $G, G^{\prime}: \mathbb{Q} \rightarrow \mathbb{R}$ are open map bisimilar. Then, the compositions $G F, G^{\prime} F^{\prime}$ : $\mathbb{P} \longrightarrow \mathbb{R}$ are open map bisimilar.

Proof: A direct proof can be found in [11]. In fact, both these results follow from the seemingly weaker Theorem 3.3 and Corollary 3.4, once we observe that the composition of profunctors preserves colimits in each argument.

(i) This can be seen by considering the coend formula for the composition of profunctors $F: \mathbb{P} \longrightarrow \mathbb{Q}$ and $G: \mathbb{Q} \rightarrow \mathbb{R}$ :

$$
G F(P, R)=\int^{Q} F(P, Q) \times G(Q, R) .
$$

The coend expression is functorial in $P$ and $R$. We might write

$$
G F=\lambda P, R . \int^{Q} F(P, Q) \times G(Q, R),
$$

a lambda expression describing $G F$ as a functor belonging to $\left[\mathbb{P} \times \mathbb{R}^{\text {op }}\right.$, Set $]$, just another way to write $\operatorname{Prof}(\mathbb{P}, \mathbb{R})$. The lambda expression exhibits the functoriality of the composition $G F$, in $F$ ranging over the category $\operatorname{Prof}(\mathbb{P}, \mathbb{Q})$ and in $G$ over the category Prof $(\mathbb{Q}, \mathbb{R})$. By inspecting the expression of the composition of $F$ and $G$ as a coend we can see that regarded as a functor in $F$ (and analogously as a functor in $G$ ) it must preserve colimits. This is because colimits of functors to cocomplete categories are obtained pointwise, coends preserve colimits (see the Appendix A.3), and, fixing 
one argument, products in Set are left adjoints, so preserve colimits. In detail, we have the following chain of isomorphisms natural in a diagram $F: \mathbb{I} \rightarrow \widehat{\mathbb{P o p} \times \mathbb{Q}}$ :

$$
\begin{aligned}
G\left(\int^{I} F(I)\right) & \cong \lambda P, R \cdot \int^{Q}\left(\int^{I} F(I)\right)(P, Q) \times G(Q, R) \\
& \cong \lambda P, R \cdot \int^{Q}\left(\int^{I} F(I)(P, Q)\right) \times G(Q, R) \text { - the colimit of } F \text { is got pointwise, } \\
& \cong \lambda P, R \cdot \int^{Q} \int^{I}(F(I)(P, Q) \times G(Q, R)) \text { as Set-product is a left adjoint, } \\
& \cong \lambda P, R \cdot \int^{I} \int^{Q}(F(I)(P, Q) \times G(Q, R)) \text { by the Fubini Theorem A.2.4, } \\
& \cong \int^{I} \lambda P, R \cdot \int^{Q}(F(I)(P, Q) \times G(Q, R)) \text { - the colimit is got pointwise, } \\
& \cong \int^{I}(G F(I))
\end{aligned}
$$

Hence, by Lemma A.10, the composition of profunctors $G F$ preserves colimits regarded as a functor in $F$ (and similarly as a functor in $G$ ). Consequently horizontal composition of 2-cells preserves openness by Theorem 3.3.

(ii) This now follows directly from Corollary 3.4.

Thus composition of profunctors preserves open maps and bisimulation in each argument. We can recover Theorem 3.3 as a special instance of Theorem 6.5. Recall the equivalence between Prof and Cocont (Proposition 4.4). To within isomorphism a colimit preserving functor can be obtained as a left Kan extension

$$
F^{\dagger}: \widehat{\mathbb{P}} \rightarrow \widehat{\mathbb{Q}}
$$

from a profunctor $F: \mathbb{P} \rightarrow \mathbb{Q}$. As observed in Propositions 4.3, 6.4, there is an open map respecting correspondence between natural transformations $\alpha: X \Rightarrow Y$ in $\widehat{\mathbb{P}}$ and 2-cells $\alpha^{\prime}: X^{\prime} \Rightarrow Y^{\prime}$ in $\operatorname{Prof}(\mathbb{1}, \mathbb{P})$. The coend definition of the horizontal composition $F \alpha^{\prime}$,

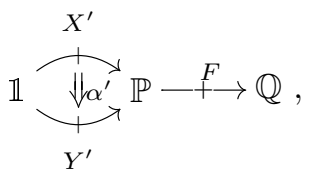

equals that of the application $F^{\dagger} \alpha$-both amount to $\int^{P} \alpha_{P} \cdot \bar{F}(P)$. In this case, that the composition of profunctors preserves open maps and bisimulation amounts to saying that $F^{\dagger}$ preserves open maps and bisimulation.

From Theorem 6.5, we obtain a characterisation of open maps between profunctors. Recall from Proposition 4.4, the correspondence to within isomorphism, between 2-cells of Prof and 2-cells of Cocont; a 2-cell $\alpha: F \Rightarrow F^{\prime}$ of Prof corresponds to a natural transformation $\alpha^{\dagger}: F^{\dagger} \Rightarrow F^{\prime \dagger}$ between colimit preserving functors.

Corollary 6.6 Let $\alpha: F \Rightarrow F^{\prime}$ be a 2 -cell between profunctors $F, F^{\prime}: \mathbb{P} \rightarrow \mathbb{Q}$. Then, $\alpha$ is open iff 
(i) the component $\alpha_{X}^{\dagger}$ is a $\mathbb{Q}$-open map, for each $X \in \widehat{\mathbb{P}}$, and

(ii) the component $\left(\alpha^{\perp}\right)_{Y}^{\dagger}$ is a $\mathbb{P}^{\text {op }}$-open map, for each $Y \in \widehat{\mathbb{Q}^{o p}}$.

Proof: 'if': Assume $\alpha_{X}^{\dagger}$ and $\left(\alpha^{\perp}\right)_{Y}^{\dagger}$ are open for any $X \in \widehat{\mathbb{P}}$ and $Y \in \widehat{\mathbb{Q}^{\circ p}}$. The correspondence $(-)^{\dagger}$ is with respect to choices of left Kan extensions, assumed to be $\left(F^{\dagger}, \theta\right)$ and $\left(F^{\prime \dagger}, \theta^{\prime}\right)$ in the cases of the profunctors $F$ and $F^{\prime}$. From the definition of $\alpha^{\dagger}$,

Hence

$$
\alpha_{\mathrm{yP}_{\mathbb{P}}^{\dagger}}^{\dagger} \theta=\theta^{\prime} \bar{\alpha} .
$$

$$
\bar{\alpha}_{P}=\theta_{P}^{\prime-1} \alpha_{\mathrm{y}_{\mathbb{P}}(P)}^{\dagger} \theta_{P},
$$

for any $P \in \mathbb{P}$. Because $\alpha_{\mathrm{y}(P)}^{\dagger}$ is open, it follows that $\bar{\alpha}_{P}$ is open, for any $P \in \mathbb{P}$. By a similar argument, from (ii) we can show that $\bar{\alpha}_{Q}^{\perp}$ is open for any $Q \in \mathbb{Q}$. Hence $\alpha$ is open by Proposition 6.2.

'only if': We consider the horizontal compositions expressed in the pictures:
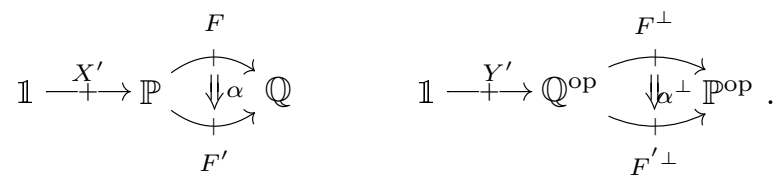

Assume $\alpha$ is open. Then so is $\alpha^{\perp}$. An application $\alpha_{X}^{\dagger}$, where $X \in \widehat{\mathbb{P}}$, equals the horizontal composition $\alpha X^{\prime}$ - both are given by the coend formula $\int^{P} X(P) . \alpha_{P}$. But the horizontal composition $\alpha X^{\prime}$ is open by Theorem 6.5. Similarly, the application of the dual $\alpha^{\perp}$ to $Y \in \widehat{\mathbb{Q}^{\text {op }}}$ equals the horizontal composition $\alpha^{\perp} Y^{\prime}$ which is again open by Theorem 6.5 .

\section{$7 \quad$ Prof and $\omega$-accessible categories}

It is often said that profunctors are to categories what relations are to sets (see e.g. [6]). In this section we pursue another analogy relating presheaf categories to non-deterministic domains, in which the presheaf construction corresponds to a powerdomain construction [21,43]. With presheaf categories as analogues of powerdomains, Prof can be regarded as a bicategory of non-deterministic domains [21].

\section{$7.1 \omega$-Accessible categories}

The operation of ideal completion, familiar in domain theory, produces an algebraic domain from a preorder (see e.g. [44]). We start with its generalisation to categories, in which a category is completed with all filtered colimits (see [33] for a discussion of filtered categories and colimits).

Definition 7.1 (Completion by filtered colimits) [35, 2] Let $\mathbb{P}$ be a small category. We write $\widetilde{\mathbb{P}}$ for the full subcategory of $\widehat{\mathbb{P}}$ consisting of presheaves whose categories of elements (see Definition A.13) are filtered. As the category of elements of each representable has a terminal object and is therefore filtered, we are justified in writing $i_{\mathbb{P}}: \mathbb{P} \rightarrow \widetilde{\mathbb{P}}$ for the functor which coincides with the Yoneda embedding. 
Proposition 7.2 For a small category $\mathbb{P}$, the category $\widetilde{\mathbb{P}}$ and embedding $i_{\mathbb{P}}$ are a free filtered colimit completion of $\mathbb{P}$. That is, $\widetilde{\mathbb{P}}$ has colimits of filtered diagrams and $i_{\mathbb{P}}: \mathbb{P} \hookrightarrow \widetilde{\mathbb{P}}$ is a functor, such that any functor $F: \mathbb{P} \rightarrow \mathcal{C}$, where $\mathcal{C}$ is a category with filtered colimits, extends to a filtered colimit preserving functor $F^{+}: \widetilde{\mathbb{P}} \rightarrow \mathcal{C}$ such that $F^{+} \circ i_{\mathbb{P}} \cong F$, unique up to a natural isomorphism:

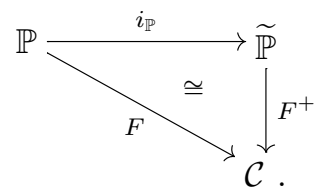

Moreover $F^{+}$is the left Kan extension $\operatorname{Lan}_{i_{\mathbb{P}}}(F)$ of $F$ along $i_{\mathbb{P}}$.

The category $\mathbf{F i l t}(\widetilde{\mathbb{P}}, \mathcal{C})$, of filtered colimit preserving functors and natural transformations, is equivalent to the functor category $\mathbf{C A T}(\mathbb{P}, \mathcal{C})$.

Proof: The proof is essentially that of [2], Theorem 2.26. It is included here for convenience, and because it sets a pattern which will recur when we consider other free completions.

The category $\widetilde{\mathbb{P}}$, as a subcategory of $\widehat{\mathbb{P}}$, is closed under filtered colimits; the category of elements of a filtered colimit of presheaves in $\widetilde{\mathbb{P}}$ may be checked to have a category of elements which is filtered.

Suppose $F: \mathbb{P} \rightarrow \mathcal{C}$ is a functor to a category $\mathcal{C}$ with all filtered colimits. Define $F^{+}$to be the functor $\operatorname{Lan}_{i_{\mathbb{P}}}(F)$ which takes $X$ in $\widetilde{\mathbb{P}}$ to the filtered colimit

$$
F^{+}(X)=\operatorname{colim}\left(\mathcal{E l}(X) \stackrel{\pi_{X}}{\longrightarrow} \mathbb{P} \stackrel{F}{\longrightarrow} \mathcal{C}\right)
$$

in $\mathcal{C}$. Because $i_{\mathbb{P}}$, which coincides with the Yoneda embedding, is full and faithful, we obtain a natural isomorphism $F^{+} \circ i_{\mathbb{P}} \cong F$, where without loss of generality we may assume that $F^{+} i_{\mathbb{P}}(P)=F(P)$.

Because colimits of presheaves are obtained pointwise, via the Yoneda Lemma, a functor $\widehat{\mathbb{P}}\left(\mathrm{y}_{\mathbb{P}}(P),-\right)$ preserves colimits. Consequently a functor $\widetilde{\mathbb{P}}\left(i_{\mathbb{P}}(P),-\right)$ preserves filtered colimits. (In other words, an object $i_{\mathbb{P}}(P)$ is finitely presentable in $\widetilde{\mathbb{P}}$.) Thus, supposing that a cone $\left\langle X_{i} \stackrel{k_{i}}{\longrightarrow} X\right\rangle_{i \in \mathbb{I}}$ is a filtered colimit, any arrow $i_{\mathbb{P}}(P) \stackrel{\bar{x}}{\longrightarrow}$ $X$, corresponding via Yoneda to an element $x \in X(P)$, will factor through some component of the cone:

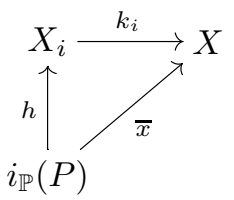

for some $i$ in $\mathbb{I}$. Hence

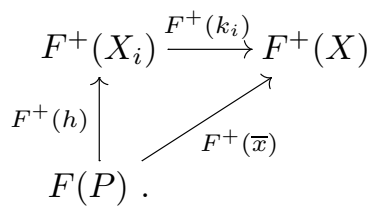


But the cone

$$
\left\langle F(P) \stackrel{F^{+}(\bar{x})}{\longrightarrow} F^{+}(X)\right\rangle_{(P, x) \in \mathcal{E} l(X)}
$$

is colimiting by definition, whence the cone

$$
\left\langle F^{+}\left(X_{i}\right) \stackrel{F^{+}\left(k_{i}\right)}{\longrightarrow} F^{+}(X)\right\rangle_{i \in \mathbb{I}}
$$

must also be colimiting. This shows that $F^{+}$preserves filtered colimits.

Any presheaf $X$ in $\mathbb{P}$ can be expressed as a filtered colimit:

$$
X \cong \operatorname{colim}\left(\mathcal{E l}(X) \stackrel{\pi_{X}}{\longrightarrow} \mathbb{P} \stackrel{i_{\mathbb{P}}}{\longrightarrow} \widetilde{\mathbb{P}}\right) \text {. }
$$

Supposing $G: \widetilde{\mathbb{P}} \rightarrow \mathcal{C}$ is filtered colimit preserving functor such that $G \circ i_{\mathbb{P}} \cong F$ ensures that

$$
G(X) \cong G\left(\operatorname{colim}\left(i_{\mathbb{P}} \circ \pi_{X}\right) \cong \operatorname{colim}\left(G \circ i_{\mathbb{P}} \circ \pi_{X}\right) \cong \operatorname{colim}\left(F \circ \pi_{X}\right) \cong F^{+}(X),\right.
$$

natural in $X$ in $\widetilde{\mathbb{P}}$.

The equivalence between the categories $\mathbf{F i l t}(\widetilde{\mathbb{P}}, \mathcal{C})$ and $\mathbf{C A T}(\mathbb{P}, \mathcal{C})$ is a consequence of Proposition A.12.

The 2-category of $\omega$-accessible categories is analogous to the category of algebraic domains and continuous functions. An $\omega$-accessible category is a free filtered colimit completion of a small category.

Definition 7.3 The 2-category $\omega$-Acc consists of

- objects: small categories, $\mathbb{P}, \mathbb{Q}, \mathbb{R}, \ldots$

- arrows: filtered colimit preserving functors between the respective filtered colimit completions i.e., $\underset{\widetilde{Q}}{F}$ is an arrow from $\mathbb{P}$ to $\mathbb{Q}$, if it is a filtered colimit preserving functor $F: \widetilde{\mathbb{P}} \rightarrow \widetilde{\mathbb{Q}}$.

- 2-cells: natural transformations between such functors.

Thus $\omega-\operatorname{Acc}(\mathbb{P}, \mathbb{Q})$ is the category $\mathbf{F i l t}(\widetilde{\mathbb{P}}, \widetilde{\mathbb{Q}})$ of filtered colimit preserving functors and natural transformations. ${ }^{4}$

We could have given an equivalent bicategorical presentation of $\omega$-Acc in terms of functors from $\mathbb{P}$ to $\widetilde{\mathbb{Q}}$ as arrows, and used the freeness property to determine the composition of arrows (just as was done for profunctors).

\subsection{Finite colimit completion}

We can exhibit Prof as a Kleisli bicategory with respect to a pseudo-monad on $\omega$-Acc. The pseudo-monad adjoins nondeterminism (it is based on the free finite colimit completion of a category) and so is analogous to a powerdomain construction, and Prof to a category of nondeterministic domains. Turning the pseudo-monad around to get a pseudo-comonad we will obtain a model of linear logic.

The constructions are based on the free completion of a (small) category under finite colimits. With the exponential of linear logic in mind [18], we write $! \mathbb{P}$ for the free finite colimit completion of $\mathbb{P}$. More exactly:

\footnotetext{
${ }^{4}$ The $\omega$ in $\omega$-Acc refers to the fact that filtered colimits are specified in terms of finite subdiagrams. For more on the notion of $\kappa$-accessible category (for $\kappa$ any regular cardinal) see [2] or [35].
} 
Definition 7.4 Let $\mathbb{P}$ be a small category. Define $! \mathbb{P}$ to be the full subcategory of $\widehat{\mathbb{P}}$ consisting of all finite colimits of representables. Write $I_{\mathbb{P}}: ! \mathbb{P} \hookrightarrow \widehat{\mathbb{P}}$ for the associated inclusion functor. Since any representable is a finite colimit of representables in an obvious way, we can write $\mathrm{y}_{\mathbb{P}}^{!}: \mathbb{P} \rightarrow ! \mathbb{P}$ for the Yoneda embedding with its codomain restricted to $! \mathbb{P}$.

Lemma 7.5 The subcategory $! \mathbb{P}$ of $\widehat{\mathbb{P}}$ is closed under all finite colimits. The category $! \mathbb{P}$ with $\mathrm{y}_{\mathbb{P}}^{!}: \mathbb{P} \rightarrow ! \mathbb{P}$ is a free finite colimit completion of $\mathbb{P}$.

Proof: The closure of $! \mathbb{P}$ under finite colimits is shown in Theorem 5.8 of [29]. The proof of freeness is straightforward.

We now show that $\left(\widehat{\mathbb{P}}, I_{\mathbb{P}}\right)$ is a free filtered colimit completion of ! $\mathbb{P}$ (see also [29], Proposition 5.41).

Theorem 7.6 The presheaf category $\widehat{\mathbb{P}}$, with $I_{\mathbb{P}}: ! \mathbb{P} \rightarrow \widehat{\mathbb{P}}$, is a free filtered colimit completion of $! \mathbb{P}$.

Proof: First note two facts concerning the presheaf images of $I_{\mathbb{P}}$.

(i) Any object $I_{\mathbb{P}}(D)$ of $\widehat{\mathbb{P}}$ is finitely presentable, i.e., $\widehat{\mathbb{P}}\left(I_{\mathbb{P}}(D),-\right.$ ) preserves filtered colimits. To see this, suppose that $D$ is a finite colimit $\int{ }^{K} \mathrm{y}_{\mathbb{P}} P_{K}$ and that $\int^{I \in \mathbb{I}} X(I)$ is a filtered colimit. Because finite limits commute with filtered colimits in Set [33], using simple coend manipulations (see Appendix A), we deduce:

$$
\begin{aligned}
\widehat{\mathbb{P}}\left(I_{\mathbb{P}}(D), \int^{I} X(I)\right) & =\widehat{\mathbb{P}}\left(\int^{K} \mathrm{y}_{\mathbb{P}} P_{K}, \int^{I} X(I)\right) \\
& \cong \int_{K} \widehat{\mathbb{P}}\left(\mathrm{y}_{\mathbb{P}} P_{K}, \int^{I} X(I)\right) \\
& \cong \int_{K} \int^{I} \widehat{\mathbb{P}}\left(\mathrm{y}_{\mathbb{P}} P_{K}, X(I)\right) \\
& \cong \int^{I} \int_{K} \widehat{\mathbb{P}}\left(\mathrm{y}_{\mathbb{P}} P_{K}, X(I)\right) \\
& \cong \int^{I} \widehat{\mathbb{P}}\left(\int^{K} \mathrm{y}_{\mathbb{P}} P_{K}, X(I)\right. \\
& =\int^{I} \widehat{\mathbb{P}}\left(I_{\mathbb{P}}(D), X(I)\right)
\end{aligned}
$$

natural in $X: \mathbb{I} \rightarrow \widehat{\mathbb{P}}$. Hence, $\widehat{\mathbb{P}}\left(I_{\mathbb{P}}(D),-\right)$ preserves filtered colimits by Lemma A.8 clearly filtered colimits are connected.

(ii) For $X$ in $\widehat{\mathbb{P}}$, the category of elements $\mathcal{E} l\left(\widehat{\mathbb{P}}\left(I_{\mathbb{P}}(-), X\right)\right)$ is filtered with $X$ the colimit of

$$
\mathcal{E} l\left(\widehat{\mathbb{P}}\left(I_{\mathbb{P}}(-), X\right)\right) \stackrel{\pi}{\longrightarrow} ! \mathbb{P} \stackrel{I_{\mathbb{P}}}{\longrightarrow} \widehat{\mathbb{P}} .
$$

This follows because, by Lemma 7.5 , objects of $! \mathbb{P}$ include the representables and are closed under finite coproducts and coequalisers. 
We now show freeness by an argument analogous to that of Proposition 7.2. The presheaf category $\widehat{\mathbb{P}}$ is closed under all colimits, so certainly under filtered colimits. Suppose $F: ! \mathbb{P} \rightarrow \mathcal{C}$ is a functor to a category with all filtered colimits. We can define the functor $F^{+}$by taking $F^{+}(X)$, for $X$ in $\widehat{\mathbb{P}}$, to be $\left(\operatorname{Lan}_{I_{\mathbb{P}}} F\right)(X)$, the colimit

$$
\operatorname{colim}\left(\mathcal{E} l\left(\widehat{\mathbb{P}}\left(I_{\mathbb{P}}(-), X\right)\right) \stackrel{\pi_{X}}{\longrightarrow} ! \mathbb{P} \stackrel{F}{\longrightarrow} \mathcal{C}\right)
$$

- the colimit is filtered by (ii).

The functor $F^{+}$is such that the triangle

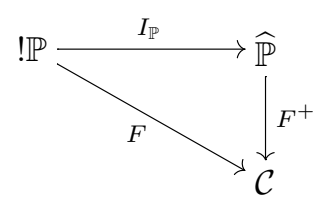

commutes up to isomorphism because $I_{\mathbb{P}}$ is full and faithful. Without loss of generality we may assume that $F^{+}\left(I_{\mathbb{P}}(D)\right)=F(D)$ for all $D$ in $! \mathbb{P}$.

The functor $F^{+}$will preserve filtered colimits because each $I_{\mathbb{P}}(D)$ is finitely presentable: Supposing $\left\langle X_{i} \stackrel{k_{i}}{\longrightarrow} X\right\rangle_{i \in \mathbb{I}}$ is a colimiting cone with $\mathbb{I}$ is filtered, any $I_{\mathbb{P}}(D) \stackrel{x}{\longrightarrow} X$ factors

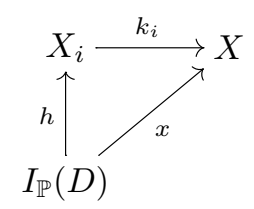

for some $i$ in $\mathbb{I}$. Hence

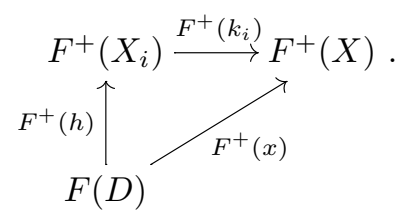

But the cone

$$
\left\langle F(P) \stackrel{F^{+}(x)}{\longrightarrow} F^{+}(X)\right\rangle_{(D, x) \in \mathcal{E} l\left(\widehat{\mathbb{P}}\left(I_{\mathbb{P}}(-), X\right)\right)}
$$

is colimiting by definition, whence the cone

$$
\left\langle F^{+} X_{i} \stackrel{F^{+} k_{i}}{\longrightarrow} F^{+} X\right\rangle_{i \in \mathbb{I}}
$$

must also be colimiting.

A filtered colimit preserving functor from $\widehat{\mathbb{P}}$ to $\mathcal{C}$ is determined to within natural isomorphism by its restriction to $! \mathbb{P}$ because, by (ii) above, every presheaf can be expressed as a filtered colimit.

Because both $\widetilde{\mathbb{P}}, i_{! \mathbb{P}}$ and $\widehat{\mathbb{P}}, I_{\mathbb{P}}$ are free filtered colimit completions, we obtain an equivalence of categories: 
Proposition 7.7 For any small category $\mathbb{P}$, there is an equivalence of categories

$$
\widetilde{\mathbb{P}} \simeq \widehat{\mathbb{P}}
$$

given by the functors

$$
\operatorname{Lan}_{i ! \mathbb{P}}\left(I_{\mathbb{P}}\right): \widetilde{\mathbb{P}} \rightarrow \widehat{\mathbb{P}}
$$

and

$$
\operatorname{Lan}_{I_{\mathbb{P}}}\left(i_{! \mathbb{P}}\right): \widehat{\mathbb{P}} \rightarrow \widetilde{\mathbb{P}} .
$$

The functor, $\operatorname{Lan}_{i_{\mathbb{P}}}\left(I_{\mathbb{P}}\right)$, is naturally isomorphic to the functor $Y \mapsto \int^{D} Y(D) \cdot I_{\mathbb{P}}(D)$. The functor, $\operatorname{Lan}_{I_{\mathbb{P}}}\left(i_{! \mathbb{P}}\right)$, is naturally isomorphic to the functor $I_{\mathbb{P}}^{*}: X \mapsto \widehat{\mathbb{P}}\left(I_{\mathbb{P}}(-), X\right)$.

Proof: The equivalence and functors establishing it are given by Proposition 7.2 and (the proof of) Theorem 7.6. As noted in Appendix A.4.2, the application of a pointwise left Kan extension may be expressed as a coend. In particular, $\operatorname{Lan}_{I_{\mathbb{P}}}\left(i_{! \mathbb{P}}\right)(X)$, where $X$ is presheaf over $\mathbb{P}$, may be expressed as the coend

$$
\int^{D} \widehat{\mathbb{P}}\left(I_{\mathbb{P}}(D), X\right) \cdot \mathrm{y} ! \mathbb{P}(D) \cong \widehat{\mathbb{P}}\left(I_{\mathbb{P}}(-), X\right)
$$

using the density formula (Appendix A.4.4). Similarly, for $Y$ in $\widetilde{\mathbb{P}}$,

$$
\operatorname{Lan}_{i_{! \mathbb{P}}}\left(I_{\mathbb{P}}\right)(Y) \cong \int^{D} \widetilde{\mathbb{P}}\left(i_{! \mathbb{P}}(D), Y\right) \cdot I_{\mathbb{P}}(D) \cong \int^{D} Y(D) \cdot I_{\mathbb{P}}(D),
$$

by the Yoneda Lemma.

The proof of Theorem 7.6 above shows us how to represent filtered colimit preserving functors between presheaf categories as profunctors.

Proposition 7.8 For any two small categories $\mathbb{P}$ and $\mathbb{Q}$ there is an equivalence of categories

$$
\operatorname{Prof}(! \mathbb{P}, \mathbb{Q}) \simeq \operatorname{Filt}(\widehat{\mathbb{P}}, \widehat{\mathbb{Q}})
$$

given by $F \mapsto \operatorname{Lan}_{I_{\mathbb{P}}}(\bar{F})$ for $F$ in $\operatorname{Prof}(! \mathbb{P}, \mathbb{Q})$, and $G \mapsto \overline{G \circ I_{\mathbb{P}}}$ for $G$ in $\mathbf{F i l t}(\widehat{\mathbb{P}}, \widehat{\mathbb{Q}})$.

Proof: From the proof of freeness, Theorem 7.6 above, a profunctor $F: ! \mathbb{P} \mapsto \rightarrow Q$ gives rise to $\operatorname{Lan}_{I_{\mathbb{P}}}(\bar{F}): \widehat{\mathbb{P}} \rightarrow \widehat{\mathbb{Q}}$, a filtered colimit preserving functor, unique up to isomorphism such that $\operatorname{Lan}_{I_{\mathbb{P}}}(\bar{F}) \circ I_{\mathbb{P}} \cong F$. The equivalence is a direct consequence of Proposition A.12 as $I_{\mathbb{P}}$ is full and faithful.

Consequently, for any small categories $\mathbb{P}$ and $\mathbb{Q}$ there is an equivalence

$$
\operatorname{Cocont}(! \mathbb{P}, \mathbb{Q}) \simeq \omega-\operatorname{Acc}(\mathbb{P}, \mathbb{Q}) .
$$

This is part of a pseudo-adjunction. We recall one way of presenting a pseudoadjunction between 2-categories from [45].

Definition 7.9 Let $\mathcal{C}$ and $\mathcal{D}$ be 2-categories. A left pseudo-adjoint to a 2-functor $U: \mathcal{C} \rightarrow \mathcal{D}$ is given by, for each object $X$ of $\mathcal{D}$, a 1-cell $\eta_{X}: X \rightarrow U F X$ in $\mathcal{D}$ such that the composition with $\eta_{X}$ induces an equivalence of categories from $\mathcal{C}(F X, Y)$ to $\mathcal{D}(X, U Y)$ for any object $Y$ of $\mathcal{C}$. 
Proposition 7.10 For any two small categories $\mathbb{P}$ and $\mathbb{Q}$ there is an equivalence of categories

$$
\operatorname{Cocont}(! \mathbb{P}, \mathbb{Q}) \simeq \operatorname{Filt}(\widehat{\mathbb{P}}, \widehat{\mathbb{Q}})
$$

induced by composition with $I_{\mathbb{P}}^{*}: X \mapsto \widehat{\mathbb{P}}\left(I_{\mathbb{P}}(-), X\right)$.

There is a pseudo-adjunction in which! together with $I_{\mathbb{P}}^{*}: \mathbb{P} \rightarrow ! \mathbb{P}$ in $\omega$-Acc is a left pseudo-adjoint to the inclusion 2-functor from Cocont to $\omega$-Acc.

Proof: Composing equivalences

$$
\operatorname{Cocont}(! \mathbb{P}, \mathbb{Q}) \simeq \operatorname{Prof}(! \mathbb{P}, \mathbb{Q}) \simeq \operatorname{Filt}(\widehat{\mathbb{P}}, \widehat{\mathbb{Q}})
$$

from Propositions 4.4 and 7.8 , we obtain an equivalence from $\operatorname{Cocont}(! \mathbb{P}, \mathbb{Q})$ to Filt $(\widehat{\mathbb{P}}, \widehat{\mathbb{Q}})$; it takes $G: ! \mathbb{P} \rightarrow \mathbb{Q}$ in Cocont to $\operatorname{Lan}_{I_{\mathbb{P}}}\left(\overline{G \circ I_{\mathbb{P}}}\right)$. Moreover it is induced by composition with $I_{\mathbb{P}}^{*}$, as

$$
\operatorname{Lan}_{I_{\mathbb{P}}}\left(\overline{G \circ I_{\mathbb{P}}}\right) \cong \operatorname{Lan}_{\mathrm{y} ! \mathbb{P}}\left(G \circ I_{\mathbb{P}}\right) \circ I_{\mathbb{P}}^{*} \cong G \circ I_{\mathbb{P}}^{*},
$$

using the factorisation of left Kan extensions in Lemma A.14.

The characterisation in Proposition 7.7 of $I_{\mathbb{P}}^{*}$ shows it to be filtered colimit preserving and so 1-cell in $\omega$-Acc. This makes ! together with $I_{\mathbb{P}}^{*}$ to be a left pseudo-adjoint to the inclusion functor.

It follows that ! extends to a pseudo-functor in a pseudo-adjunction:

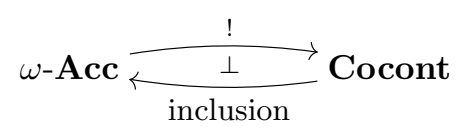

The pseudo-functor !, post-composed with the inclusion 2-functor to form a 2functor on $\omega$-Acc, can be equipped with multiplication, unit and corresponding coherence modifications in order to form a pseudo-monad [16] (a doctrine in the terminology of [48]). The bicategory of its free algebras, the Kleisli bicategory for !, is biequivalent to Prof, and Cocont.

In computational terms, the effect of ! of the pseudo-monad is to adjoin nondeterminism. This is traditionally achieved in domain theory by using powerdomains; adjoining non-determinism to a "domain" $\widetilde{\mathbb{P}}$, with basis the small category $\mathbb{P}$, produces the "non-deterministic domain" $\widetilde{\mathbb{P}}$, equivalent to $\widehat{\mathbb{P}}$. We can view Prof as a bicategory of "non-deterministic domains" analogous to the Kleisli category of a powerdomain.

If we "turn around" the pseudo-monad (and look instead at the pre-composition of ! with the inclusion 2-functor above), we obtain a pseudo-comonad on Cocont, and so on Prof, that we also denote by !. Its coKleisli bicategory is biequivalent to the 2-category with small categories as objects, 1-cells $F: \mathbb{P} \rightarrow \mathbb{Q}$ being filtered colimit preserving functors $F: \widehat{\mathbb{P}} \rightarrow \widehat{\mathbb{Q}}$ and 2-cells natural transformations. The pseudo-comonad ! can play the role of the "exponential" of linear logic and is one of several ways in which to adjoin a pseudo-comonad to Prof, so obtaining what can be viewed as a (bi)categorical model of Girard's classical linear logic [47]. (It constitutes the basic prefixing operation in the presheaf semantics of the higher order process language HOPLA [40].) 


\subsubsection{Domain theoretic analogies}

Analogous results are familiar in domain theory. Perhaps the closest analogue is obtained by replacing small categories $\mathbb{P}, \mathbb{Q}, \ldots$ by partial orders, presheaf categories by domains of downwards closed subsets ordered by inclusion, colimits by least upper bounds (with lubs given by unions) and filtered diagrams by directed subsets.

Then $\omega$-Acc would be replaced by continuous functions between ideal completions of partial orders (a category of algebraic cpos), and Cocont by additive (i.e., lub preserving) functions between domains of downwards closed subsets (a category of prime algebraic lattices). Now an additive function from a $\widehat{\mathbb{P}}$ to a $\widehat{\mathbb{Q}}$ can be represented by a monotonic function from the partial order $\mathbb{P}$ to $\widehat{\mathbb{Q}}$, or equivalently as a "relation", a downwards closed subset of $\mathbb{P}^{o p} \times \mathbb{Q}$ - a direct analogue of a profunctor, in which the category Set is replaced by the partial order $\emptyset \subseteq 1$.

In this domain set-up, we can take $! \mathbb{P}$ to be the finite lub completion of a partial order $\mathbb{P}$ (equivalently, the order got by restricting $\widehat{\mathbb{P}}$ to its finite elements). The analogue of the pseudo-monad above would be the monad associated with the lower (or Hoare) powerdomain, which given an ideal completion $\widetilde{\mathbb{P}}$ of a partial order $\mathbb{P}$ returns $\widetilde{\mathbb{P}}$, the ideal completion of $! \mathbb{P}$, that is isomorphic to $\widehat{\mathbb{P}}$.

The analogue of the pseudo-comonad would be the comonad on the category of prime algebraic lattices with additive functions given by !; the co-Kleisli category of the comonad would be equivalent to that of continuous functions between prime algebraic lattices, expressing the well known fact that a continuous function is determined by its restriction to just the finite elements. (See [40] for more details.)

An attractive feature of the pseudo-comonad !, freely adjoining finite colimits, is that it generalises a situation in traditional domain theory. However, as we shall see, there are other considerations, to do with how well bisimulation is respected, that argue for alternatives to this choice of comonad.

\subsection{A failure of open map preservation}

We have seen how Prof and the pseudo-comonad!, which on a small category yields its finite colimit completion, are sufficiently rich in structure that they can be regarded as a model of classical linear logic. The results of Section 6 say that the model's linear arrows, those in Prof, preserve open map bisimulation. A typical arrow in the coKleisli bicategory of ! is a profunctor

$$
F: ! \mathbb{P} \rightarrow \mathbb{Q} .
$$

It corresponds to a filtered colimit preserving functor

$$
\operatorname{Lan}_{I \mathbb{P}}(\bar{F}): \widehat{\mathbb{P}} \rightarrow \widehat{\mathbb{Q}}
$$

between presheaf categories, by Proposition 7.8 , where $I_{\mathbb{P}}$ is the embedding of $! \mathbb{P}$ into $\widehat{\mathbb{P}}$. Prima facie it might be hoped that $\operatorname{Lan}_{I_{\mathrm{P}}}(\bar{F})$ preserved open map bisimulation; that an open map in $\widehat{\mathbb{P}}$ was sent to an open map in $\widehat{\mathbb{Q}}$. Indeed, if we weaken open maps in $\widehat{\mathbb{P}}$, by convention understood to be with respect to the Yoneda embedding $\mathrm{y}_{\mathbb{P}}: \mathbb{P} \hookrightarrow \widehat{\mathbb{P}}$, to open maps with respect to the inclusion $I_{\mathbb{P}}: ! \mathbb{P} \hookrightarrow \widehat{\mathbb{P}}$, we can obtain a preservation result as a consequence of the following factorisation: 


$$
\operatorname{Lan}_{I_{\mathbb{P}}}(\bar{F}) \cong \operatorname{Lan}_{\mathrm{y}}(\bar{F}) \circ I_{\mathbb{P}}^{*}
$$

- this is a special case of Proposition A.14 in the Appendix. It follows that $\operatorname{Lan}_{I_{\mathbb{P}}}$

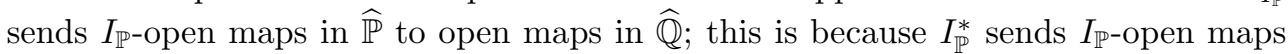
to $\mathrm{y} ! \mathbb{P}$-open maps (by Lemma 2.6).

But, unfortunately, $I_{\mathbb{P}}$-bisimulation degenerates to isomorphism:

Proposition 7.11 Let $X$ and $Y$ be presheaves in $\widehat{\mathbb{P}}$. Then, $X$ and $Y$ are $I_{\mathbb{P}}$-bisimilar iff $X$ and $Y$ are isomorphic presheaves.

Proof: We show that the isomorphisms are the only surjective $I_{\mathbb{P} \text {-open maps between }}$ presheaves over $\mathbb{P}$. Let $f: X \rightarrow Y$ be a surjective $I_{\mathbb{P}^{-} \text {open map. By definition it }}$ is an epimorphism. To show that $f$ is an isomorphism it is now enough to show that $f$ is a monomorphism as well (see [34]). Since $f$ is a natural transformation between presheaves, $f$ is a monomorphism iff for every object $P$ of $\mathbb{P}$, the function $f_{P}: X(P) \rightarrow Y(P)$ is injective. Suppose then that $x, x^{\prime} \in X(P)$ are such that $f_{P}(x)=f_{P}\left(x^{\prime}\right)$. Via the Yoneda lemma, we then have that the following square commutes:

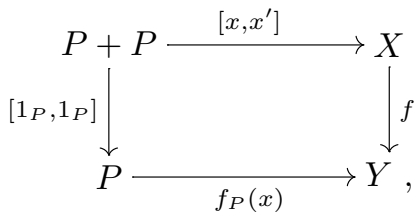

where we have let objects of $\mathbb{P}$ stand for their corresponding representables and elements of $X$ for the corresponding arrows to $X$. Since $f$ is $I_{\mathbb{P}}$-open and $P+P$, as well as $P$ are in $! P$, there exists $x^{\prime \prime}: P \rightarrow X$ such that $x^{\prime \prime} \circ\left[1_{P}, 1_{P}\right]=\left[x, x^{\prime}\right]$. Since $x^{\prime \prime} \circ\left[1_{P}, 1_{P}\right]=\left[x^{\prime \prime}, x^{\prime \prime}\right]$, we can conclude that $x=x^{\prime}$.

The arrows in the co-Kleisli bicategory of ! are too liberal to ensure preservation of more than the most trivial bisimulation! This negative result is backed up by examples where bisimilarity is not preserved by arrows in the co-Kleisli bicategory, corresponding to filtered colimit preserving functors. It is not hard to cook up an example of a filtered colimit preserving functor which sends the domain and codomain of a surjective open map to two non-bisimilar objects; for example, where the functor goes from $\widehat{\mathbb{1}}$, i.e. Set, to "synchronisation trees", i.e. presheaves over the partial order category of non-empty strings.

Remark: Observe that in order for the argument of Proposition 7.11 to go through it is enough to assume that the arrow $\left[1_{P}, 1_{P}\right]: P+P \rightarrow P$ from the coproduct of representables $P+P$ lies in $! \mathbb{P}$.

These results suggest that we look for alternative pseudo-comonads on Cocont and its equivalent Prof, where in expanding the arrows to those in the co-Kleisli bicategory, we do not lose preservation of open map bisimulation.

\section{$8 \quad$ Lifting and connected colimits}

Our next example of a pseudo-comonad is provided by the lifting operation on Prof. Its co-Kleisli bicategory provides a model of affine linear logic [26]. Arrows in the 
co-Kleisli bicategory will correspond to connected colimit preserving functors between presheaf categories. Such functors do not have to send the empty presheaf to the empty presheaf, but will still preserve open map bisimulation. This relaxation makes the category of connected colimit preserving functors between presheaf categories a suitable framework in which to give semantics to a wide range of process languages $[52$, $11,39]$.

\subsection{Lifting}

Definition 8.1 (Lifting) Define $(-)_{\perp}:$ Prof $\rightarrow$ Prof to be the following pseudofunctor:

- On objects: $\mathbb{P}_{\perp}$ is the category $\mathbb{P}$ to which it has been added a new strict initial object, often referred to as $\perp$. The objects of $\mathbb{P}_{\perp}$ other than $\perp$ are often written $\lfloor P\rfloor$ for $P$ an object of $\mathbb{P}$.

- On arrows: If $F: \mathbb{P} \longrightarrow \mathbb{Q}, F_{\perp}$ is defined by:

$$
F_{\perp}\left(P^{\prime}, Q^{\prime}\right)=\left\{\begin{array}{cl}
F(P, Q) & \text { if } P^{\prime}=\lfloor P\rfloor \text { and } Q^{\prime}=\lfloor Q\rfloor, \\
\{*\} & \text { if } Q^{\prime}=\perp \\
\emptyset & \text { otherwise. }
\end{array}\right.
$$

- On 2-cells: $A$ 2-cell $\alpha: F \Rightarrow G$ is extended with identity functions for the extra components to cover the new cases.

Not only is $\widehat{\mathbb{P}}, \mathrm{y}_{\mathbb{P}}$ a free colimit completion of $\mathbb{P}$, but also, as we will see shortly, $\widehat{\mathbb{P}}, \mathrm{j}_{\mathbb{P}_{\perp}}$ is a free connected colimit completion of $\mathbb{P}_{\perp}$, where $\mathrm{j}_{\mathbb{P}_{\perp}}: \mathbb{P}_{\perp} \rightarrow \widehat{\mathbb{P}}$ is the strict Yoneda embedding, now defined.

Definition 8.2 Writing $l: \mathbb{P} \rightarrow \mathbb{P}_{\perp}$, for the "inclusion" functor $P \mapsto\lfloor P\rfloor$, from a small category $\mathbb{P}$, in Cat, the construction $\mathbb{P}_{\perp}, l$ freely adjoins an initial object (in other words, it is the free completion of $\mathbb{P}$ with the colimit of the empty diagram). This freeness yields a unique initial-object preserving functor

$$
\mathrm{j}_{\mathbb{P}_{\perp}}: \mathbb{P}_{\perp} \rightarrow \widehat{\mathbb{P}}
$$

such that

$$
\mathrm{j}_{\mathbb{P}_{\perp}} \circ l=\mathrm{y}_{\mathbb{P}} .
$$

The functor $\mathrm{j}_{\mathbb{P}_{\perp}}$ sends every non-initial object to the corresponding representable and the initial object $\perp$ to the empty presheaf, the initial object of $\widehat{\mathbb{P}}$.

Associated with $\mathrm{j}_{\mathbb{P}_{\perp}}$ is the functor $\mathrm{j}_{\mathbb{P}_{\perp}}^{*}: \widehat{\mathbb{P}} \rightarrow \widehat{\mathbb{P}_{\perp}}$, which takes a presheaf $X$ in $\widehat{\mathbb{P}}$ to the presheaf $\widehat{\mathbb{P}}\left(\mathrm{j}_{\mathbb{P}_{\perp}}(-), X\right)$ in $\widehat{\mathbb{P}_{\perp}}$. The presheaf $\mathrm{j}_{\mathbb{P}_{\perp}}^{*}(X)$ is such that

$$
\mathrm{j}_{\mathbb{P}_{\perp}}^{*}(X)(\lfloor P\rfloor)=\widehat{\mathbb{P}}\left(j_{\mathbb{P}_{\perp}}\lfloor P\rfloor, X\right)=\widehat{\mathbb{P}}\left(\mathrm{y}_{\mathbb{P}} P, X\right) \cong X(P)
$$

and

$$
\mathrm{j}_{\mathbb{P}_{\perp}}^{*}(X)(\perp)=\widehat{\mathbb{P}}\left(j_{\mathbb{P}_{\perp}} \perp, X\right)=\widehat{\mathbb{P}}(\emptyset, X) \text {, a singleton set. }
$$

Notation: We write $\lfloor-\rfloor$ for the functor $\mathrm{j}_{\mathbb{P}_{\perp}}^{*}(-)$. 
Thus, the functor $\lfloor-\rfloor: \widehat{\mathbb{P}} \rightarrow \widehat{\mathbb{P}_{\perp}}$ has a simple description; it adjoins a "root" to a presheaf $X$ in $\widehat{\mathbb{P}}$ in the sense that $\lfloor X\rfloor(\lfloor P\rfloor)$ is a copy of $X(P)$ for any $P$ in $\mathbb{P}$, while $\lfloor X\rfloor(\perp)$ is singleton set $\{*\}$, the new root being $*$. Presheaves that to within isomorphism can be obtained in this way are called rooted in [28]. Any presheaf in $\widehat{\mathbb{P}_{\perp}}$ has an essentially unique decomposition as a coproduct of rooted presheaves - its rooted decomposition:

Proposition 8.3 Let $Y \in \widehat{\mathbb{P}_{\perp}}$. Then,

$$
Y \cong \sum_{i \in Y(\perp)}\left\lfloor Y_{i}\right\rfloor
$$

where, for $i \in Y(\perp)$, the presheaf $Y_{i}$ in $\widehat{\mathbb{P}}$ is the restriction of $Y$ to the elements over $P$, an object of $\mathbb{P}$, which $Y$ sends to $i$, viz.

$$
Y_{i}(P)=\{x \in Y(\lfloor P\rfloor) \mid Y(u)(x)=i\}
$$

—we have written $u: \perp \rightarrow\lfloor P\rfloor$ for the unique map in $\mathbb{P}_{\perp}$ from the initial object.

\subsection{Connected colimit preserving functors}

In Section 7 it was shown how to represent filtered colimit preserving functors between presheaf categories in Prof using a comonad, !. We now concentrate on another class of functors that we have found prevalent in the semantics of processes, this time based on lifting. These are functors which preserve connected colimits. A colimit is connected when its diagram is nonempty and connected as a graph [41]. Using lifting, we can describe connected colimit preserving functors between presheaf categories as certain arrows in Prof.

Proposition 8.4 The functor $\lfloor-\rfloor: \widehat{\mathbb{P}} \rightarrow \widehat{\mathbb{P}}$ preserves connected colimits.

Proof: Let $\mathbb{K}$ be a connected category. In order to apply Lemma A.8, we should check that

$$
\left\lfloor\int^{K} X(K)\right\rfloor\left(P^{\prime}\right) \cong\left(\int^{K}\lfloor X(K)\rfloor\right)\left(P^{\prime}\right)
$$

holds, and is natural in $X: \mathbb{K} \rightarrow \widehat{\mathbb{P}}$ and $P^{\prime} \in \mathbb{P}_{\perp}$.

In the case where $P^{\prime}=\lfloor P\rfloor$ for $P$ in $\mathbb{P}$, the isomorphism and its naturality in $X$ and $P$ follow by the Yoneda lemma and because colimits of presheaves are got pointwise:

$$
\begin{aligned}
\left.\int^{K} X(K)\right\rfloor(\lfloor P\rfloor) & =\widehat{\mathbb{P}}\left(j_{\mathbb{P}_{\perp}}\lfloor P\rfloor, \int^{K} X(K)\right) \\
& =\widehat{\mathbb{P}}\left(\mathrm{y}_{\mathbb{P}} P, \int^{K} X(K)\right) \\
& \cong\left(\int^{K} X(K)\right)(P) \\
& \cong \int^{K}(X(K)(P))
\end{aligned}
$$




$$
\begin{aligned}
& =\int^{K}(\lfloor X(K)\rfloor(\lfloor P\rfloor)) \\
& \cong\left(\int^{K}\lfloor X(K)\rfloor\right)(\lfloor P\rfloor),
\end{aligned}
$$

all of which isomorphisms are natural in $X$ and $P$. In the case where $P^{\prime}=\perp$, the isomorphism follows because a colimit of connected singletons is a singleton. It is then easy to show naturality in $P^{\prime}$ throughout $\mathbb{P}_{\perp}$ by exhibiting the additional naturality squares associated with arrows $\perp \rightarrow\lfloor P\rfloor$.

Proposition 8.5 The presheaf category $\widehat{\mathbb{P}}$, with $\mathrm{j}_{\perp}: \mathbb{P}_{\perp} \rightarrow \widehat{\mathbb{P}}$, is a free connected colimit completion of $\mathbb{P}_{\perp}$.

Proof: To show freeness, suppose $F: \mathbb{P}_{\perp} \rightarrow \mathcal{C}$ is a functor to a category with all connected colimits. Define the left Kan extension $\operatorname{Lan}_{\mathrm{jp}_{\perp}} F$ by

$$
\left(\operatorname{Lan}_{\mathbb{j}_{\perp}} F\right)(X)=\operatorname{colim}\left(\mathcal{E} l(\lfloor X\rfloor) \stackrel{\pi_{X}}{\longrightarrow} \mathbb{P}_{\perp} \stackrel{F}{\longrightarrow} \mathcal{C}\right),
$$

for $X$ in $\widehat{\mathbb{P}}$; clearly the category of elements of the rooted presheaf $\lfloor X\rfloor$ has an initial element at $\perp$ and so is connected.

Because $j_{\mathbb{P}_{\perp}}$ is full and faithful we have that

$$
\left(\operatorname{Lan}_{\mathrm{jp}_{\perp}} F\right) \circ \mathrm{j}_{\mathbb{P}_{\perp}} \cong F
$$

Abbreviate $\operatorname{Lan}_{\mathrm{j}_{\perp}} F$ to $F^{+}$. Without loss of generality we may assume that $F^{+} \mathrm{j}_{\mathbb{P}_{\perp}}(P)=F(P)$ for all $P$ in $\mathbb{P}_{\perp}$. To see that $F^{+}$preserves connected colimits, let $\left\langle X_{i} \stackrel{k_{i}}{\longrightarrow} X\right\rangle_{i \in \mathbb{I}}$ be a colimiting cone with $\mathbb{I}$ connected. Any $x: \mathrm{j}_{\mathbb{P}_{\perp}}(P) \rightarrow X$, with $P$ in $\mathbb{P}_{\perp}$, must factor

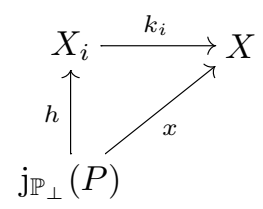

for some $I$ in $\mathbb{I}$. Hence

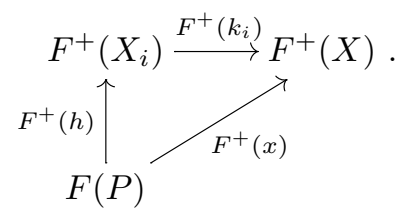

But the cone

$$
\left\langle F(P) \stackrel{F^{+}(x)}{\longrightarrow} F^{+}(X)\right\rangle_{(P, x) \in \mathcal{E} l(\lfloor X\rfloor)}
$$

is colimiting by definition, whence the cone

$$
\left\langle F^{+} X_{i} \stackrel{F^{+} k_{i}}{\longrightarrow} F^{+} X\right\rangle_{i \in \mathbb{I}}
$$

must also be colimiting. 
Thus $F^{+}$is connected colimit preserving and satisfies $F^{+} \circ \mathrm{j}_{\mathbb{P}_{\perp}} \cong F$. These properties determine $F^{+}$to within natural isomorphism, as we now show.

Any presheaf $X$ in $\widehat{\mathbb{P}}$ can be expressed as a connected colimit:

$$
X \cong \operatorname{colim}\left(\mathcal{E} l(\lfloor X\rfloor) \stackrel{\pi_{X}}{\longrightarrow} \mathbb{P}_{\perp} \stackrel{\mathrm{j}_{\perp}}{\longrightarrow} \widehat{\mathbb{P}}\right) .
$$

Hence, supposing that $G: \widehat{\mathbb{P}} \rightarrow \mathcal{C}$ is connected colimit preserving such that $G \circ j_{\mathbb{P}_{\perp}} \cong F$ ensures that $G(X) \cong F^{+}(X)$, natural in $X$.

Definition 8.6 The 2-category Conn consists of all small categories as objects, with arrows from $\mathbb{P}$ to $\mathbb{Q}$ being the connected colimit preserving functors from $\widehat{\mathbb{P}}$ to $\widehat{\mathbb{Q}}$, and 2-cells the natural transformations between such functors.

Proposition 8.7 There is an equivalence of categories

$$
\operatorname{Prof}\left(\mathbb{P}_{\perp}, \mathbb{Q}\right) \simeq \operatorname{Conn}(\mathbb{P}, \mathbb{Q}),
$$

for any two small categories $\mathbb{P}$ and $\mathbb{Q}$.

The functors exhibiting the equivalence are

$$
F \mapsto \operatorname{Lan}_{\mathrm{jP}_{\perp}} \bar{F}
$$

from $\operatorname{Prof}\left(\mathbb{P}_{\perp}, \mathbb{Q}\right)$ to $\operatorname{Conn}(\mathbb{P}, \mathbb{Q})$, and

$$
G \mapsto \overline{G \circ \mathrm{j}_{\perp}}
$$

from $\operatorname{Conn}(\mathbb{P}, \mathbb{Q})$ to $\operatorname{Prof}\left(\mathbb{P}_{\perp}, \mathbb{Q}\right)$.

Proof: That the two functors above are mutual inverses to within natural isomorphism follows directly from $\widehat{\mathbb{P}}$ being the free connected colimit completion of $\mathbb{P}_{\perp}$, Proposition 8.5.

The above proposition is really part of a pseudo-adjunction which we most easily express using Cocont in place of Prof. The inclusion 2-functor from Cocont to Conn has a left pseudo-adjoint, the operation of lifting $(-)_{\perp}$ extended to 2 -functor from Conn to Cocont. The definition of lifting as a 2 -functor relies on the rooted decomposition of presheaves - see Proposition 8.3

Let $Y$ and $Z$ be presheaves in $\widehat{\mathbb{P}_{\perp}}$ with rooted decompositions $Y \cong \sum_{i \in Y(\perp)}\left\lfloor Y_{i}\right\rfloor$ and $Z \cong \sum_{j \in Z(\perp)}\left\lfloor Z_{j}\right\rfloor$. A map of presheaves $f: Y \rightarrow Z$ in $\widehat{\mathbb{P}_{\perp}}$ also decomposes:

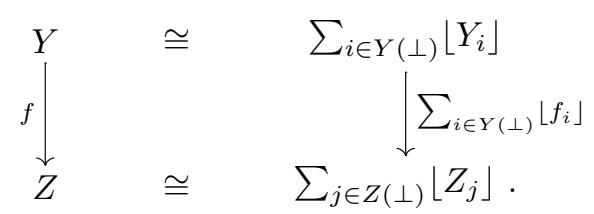

By naturality, for each $i \in Y(\perp), f$ restricts to a map $f_{i}: Y_{i} \rightarrow Z_{f_{\perp}(i)}$ in $\widehat{\mathbb{P}}$. The function $f_{\perp}: Y(\perp) \rightarrow Z(\perp)$ expresses to which components of $Z$ the components of $Y$ are sent.

Via the rooted decomposition of presheaves over lifted categories we can express lifting as a 2-functor from Conn to Cocont. 
Definition 8.8 Define the 2-functor $(-)_{\perp}:$ Conn $\rightarrow$ Cocont to act as follows.

- On objects: An object $\mathbb{P}$ is sent to $\mathbb{P}_{\perp}$, in which an initial object $\perp$ has been adjoined freely to $\mathbb{P}$.

- On arrows: Let $F: \mathbb{P} \rightarrow \mathbb{Q}$ be an arrow in Conn. The functor $F_{\perp}: \mathbb{P}_{\perp} \rightarrow \mathbb{Q}_{\perp}$ takes an arrow $f: Y \rightarrow Z$ with decomposition

$$
\sum_{i \in Y(\perp)}\left\lfloor f_{i}\right\rfloor: \sum_{i \in Y(\perp)}\left\lfloor Y_{i}\right\rfloor \rightarrow \sum_{j \in Z(\perp)}\left\lfloor Z_{j}\right\rfloor
$$

to the arrow

$$
\sum_{i \in Y(\perp)}\left\lfloor F\left(f_{i}\right)\right\rfloor: \sum_{i \in Y(\perp)}\left\lfloor F\left(Y_{i}\right)\right\rfloor \rightarrow \sum_{j \in Z(\perp)}\left\lfloor F\left(Z_{j}\right)\right\rfloor .
$$

- On 2-cells: A 2-cell $\alpha: F \Rightarrow G$ is sent to the 2-cell $\alpha_{\perp}: F_{\perp} \Rightarrow G_{\perp}$, a natural transformation with components

$$
\left(\alpha_{\perp}\right)_{Y}=\sum_{i \in Y(\perp)}\left\lfloor\alpha_{Y_{i}}\right\rfloor: \sum_{i \in Y(\perp)}\left\lfloor F\left(Y_{i}\right)\right\rfloor \rightarrow \sum_{i \in Y(\perp)}\left\lfloor G\left(Y_{i}\right)\right\rfloor,
$$

at $Y$ a presheaf in $\widehat{\mathbb{P}_{\perp}}$.

The 2-functor $(-)_{\perp}$ is a left pseudo-adjoint to the inclusion 2-functor from Cocont to Conn.

Proposition 8.9 Composition with $\lfloor-\rfloor: \widehat{\mathbb{P}} \rightarrow \widehat{\mathbb{P}_{\perp}}$ induces an equivalence of categories

$$
\operatorname{Cocont}\left(\mathbb{P}_{\perp}, \mathbb{Q}\right) \simeq \operatorname{Conn}(\mathbb{P}, \mathbb{Q}) .
$$

There is a pseudo-adjunction in which $(-)_{\perp}$ together with $\left\lfloor-_{-}\right\rfloor$is a left pseudo-adjoint to the inclusion 2-functor from Cocont to Conn:

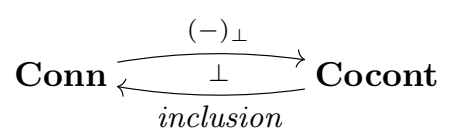

Proof: Similar to that of Proposition 7.10.

The pseudo-adjunction induces a pseudo-comonad on Cocont. Its coKleisli bicategory, biequivalent to Conn, is not cartesian closed, but can be viewed as a model of affine linear logic $[26,40]$.

\subsubsection{Rooted colimits}

Although the results of this section are phrased in terms of connected colimits we could equally well have replaced their use by special connected colimits which we call "rooted".

Definition 8.10 A diagram in a category $\mathcal{C}$ is said to be rooted iff it is a functor $\mathbb{I}_{\perp} \rightarrow \mathcal{C}$, for $\mathbb{I}$ a small category. A colimit is rooted iff its diagram is rooted.

Proposition 8.11 A category is cocomplete iff it has an initial object and all rooted colimits. 
Proof: "If": Any diagram $\mathbb{I} \rightarrow \mathcal{C}$ extends to a rooted diagram $\mathbb{I}_{\perp} \rightarrow \mathcal{C}$ in which $\perp$ is sent to the initial object. The colimiting cone for the rooted diagram restricts to a colimiting cone for the original diagram. "Only if": trivial.

In particular, as we have seen, the free connected colimit completion of $\mathbb{P}_{\perp}$ is $\widehat{\mathbb{P}}$ which has all colimits; because $\mathbb{P}_{\perp}$ has an initial object so must the completion have an initial object in addition to all connected colimits.

Proposition 8.12 Assume that $\mathcal{C}$ is a cocomplete category. A functor $F: \mathcal{C} \rightarrow \mathcal{D}$ preserves connected colimits iff it preserves rooted colimits.

Proof: "Only if": Trivial, as a rooted colimit is a special kind of connected colimit. "If": Any colimiting cone from a connected diagram $\mathcal{K} \rightarrow \mathcal{C}$ extends to colimiting cone from a rooted diagram $\mathcal{K}_{\perp} \rightarrow \mathcal{C}$ in which $\perp$ is sent to the initial object. If $F$ preserves the rooted colimit it will also preserve the original connected colimit.

In particular, because presheaf categories have an initial object, functors from presheaf categories preserve connected colimits iff they preserve rooted colimits. Consequently,

Corollary 8.13 The presheaf category $\widehat{\mathbb{P}}$, with $\mathrm{j}_{\mathbb{P}_{\perp}}: \mathbb{P}_{\perp} \rightarrow \widehat{\mathbb{P}}$, is a free rooted colimit completion of $\mathbb{P}_{\perp}$.

Of course, colimit preserving functors preserve all connected colimits. Amongst the connected colimit preserving functors between presheaf categories we can easily pick out those which satisfy the stronger condition of preserving all colimits; by the next proposition, they are those functors which are strict, i.e. they send the empty presheaf to the empty presheaf.

Proposition 8.14 Assume that $\mathcal{C}$ and $\mathcal{D}$ are cocomplete categories. Suppose $F$ : $\mathcal{C} \rightarrow \mathcal{D}$ is a functor which preserves connected colimits. The following properties are equivalent:

(i) F preserves all colimits.

(ii) F preserves all coproducts.

(iii) F is strict, i.e., F preserves initial objects.

Proof: The implications (i) to (ii) and (ii) to (iii) are obvious. The implication (ii) to (i) follows because any colimit decomposes into a coproduct of connected colimits. The implication (iii) to (ii) follows because a coproduct, whose components are indexed by objects in the discrete category $\mathbb{I}$, can also be viewed as a connected colimit: the indexing is extended to $\mathbb{I}_{\perp}$, so that $\perp$ is sent to the initial object.

\subsection{Bisimulation}

We turn now to consider the preservation of bisimulation by connected colimit preserving functors. We begin with a simple but important observation.

Proposition 8.15 Let $h: X \rightarrow Y$ be a map between presheaves in $\widehat{\mathbb{P}}$. The following statements are equivalent: 
(i) The map $h$ is $j_{\mathbb{P}_{\perp}}$-open.

(ii) The map $\lfloor h\rfloor:\lfloor X\rfloor \rightarrow\lfloor Y\rfloor$ is $\mathrm{y}_{\mathbb{P}_{\perp}}$-open.

(iii) The map $h$ is surjective $\mathrm{y}_{\mathbb{P}}$-open.

Proof: By definition,

$$
\lfloor h\rfloor=j_{\mathbb{P}_{\perp}}^{*} h: j_{\mathbb{P}_{\perp}}^{*} X \rightarrow j_{\mathbb{P}_{\perp}}^{*} Y .
$$

That (i) and (ii) are equivalent is a direct consequence of Lemma 2.6.

To see the equivalence between (ii) and (iii), recall that $j_{\mathbb{P}}^{*} X=\lfloor X\rfloor$ and $j_{\mathbb{P}}^{*} Y=$ $\lfloor Y\rfloor$ are rooted presheaves, for which $\lfloor X\rfloor(\perp)$ and $\lfloor Y\rfloor(\perp)$ are singletons and $\lfloor X\rfloor(\lfloor P\rfloor) \cong$ $X(P)$ and $\lfloor Y\rfloor(\lfloor P\rfloor) \cong Y(P)$. Clearly, the square

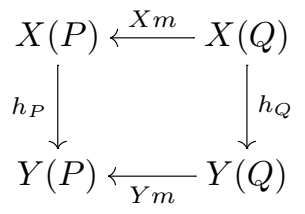

associated with $m: P \rightarrow Q$ is a quasipullback in $\widehat{\mathbb{P}}$ iff the corresponding square

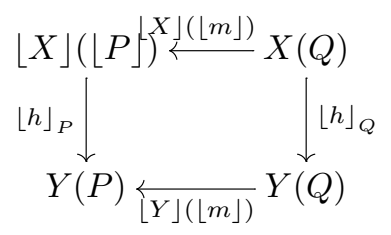

associated with $\lfloor m\rfloor:\lfloor P\rfloor \rightarrow\lfloor Q\rfloor$ is a quasipullback in $\widehat{\mathbb{P}_{\perp}}$. Letting $P$ be an object of $\mathbb{P}$, the square

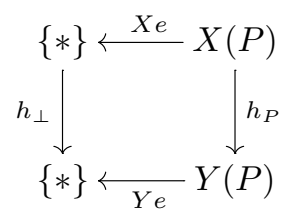

associated with the map $e: \perp \rightarrow\lfloor P\rfloor$, is a quasipullback iff $h_{P}$ is surjective.

Functors $\lfloor-\rfloor: \widehat{\mathbb{P}} \rightarrow \widehat{\mathbb{P} \perp}$ are a form of prefixing operation, as prevalent in process calculi. (Lifting constitutes the basic prefix operation in the presheaf semantics of affine HOPLA, the higher order affine language in [40], and underlies the semantics of many essentially affine process languages $[51,13,52,53]$.) They also play a key role in harnessing open map preservation in Prof to connected colimit preserving functors.

Proposition 8.16 The functor $\lfloor-\rfloor: \widehat{\mathbb{P}} \rightarrow \widehat{\mathbb{P}_{\perp}}$ preserves surjective open maps.

Proof: In relation to Lemma 2.6, we have the following situation:

$$
\mathbb{P}_{\perp} \stackrel{\mathrm{yP}_{\perp}}{\longrightarrow} \widehat{\mathbb{P}_{\perp}} \underset{\operatorname{Lan}_{\mathrm{y}_{\perp}}}{\stackrel{j_{\mathbb{P}_{\perp}}^{*}}{\longrightarrow}} \widehat{\mathbb{P}} .
$$


Notice that $\operatorname{Lan}_{\mathbb{y}_{\perp}} j_{\mathbb{P}_{\perp}} \circ \mathrm{y}_{\mathbb{P}_{\perp}} \cong j_{\mathbb{P}_{\perp}}$, because $\mathrm{y}_{\mathbb{P}_{\perp}}$ is full and faithful. Thus, by Lemma 2.6, $\lfloor-\rfloor=j_{\mathbb{P}_{\perp}}^{*}$, sends $j_{\mathbb{P}_{\perp}}$-open maps to $\mathrm{y}_{\mathbb{P}_{\perp}}$-open maps. As observed above, $j_{\mathbb{P}_{\perp}}$-open maps are the same as surjective $\mathrm{y}_{\mathbb{P}}$-open maps. Moreover, $\lfloor-\rfloor$ preserves epimorphisms as it preserves connected colimits (Proposition 8.4) and so pushouts.

We can use Corollary 3.3 to deduce the preservation of surjective open maps along connected colimit preserving functors.

Theorem 8.17 Let $G: \widehat{\mathbb{P}} \rightarrow \widehat{\mathbb{Q}}$ be a connected colimit preserving functor. Then $G$ preserves surjective open maps and open map bisimulation.

Proof: From Proposition 8.7, we know that $G \cong \operatorname{Lan}_{\mathrm{j}_{\perp}}(F)$ for some functor $F$ : $\mathbb{P}_{\perp} \rightarrow \widehat{\mathbb{Q}}$. By Proposition A.14

$$
G \cong \operatorname{Lan}_{\mathrm{j}_{\perp}} F \cong\left(\operatorname{Lan}_{\mathrm{yp}_{\mathbb{P}}} F\right) \circ \mathrm{j}_{\mathbb{P}_{\perp}}^{*}=\left(\operatorname{Lan}_{\mathrm{yP}} F\right) \circ\lfloor-\rfloor .
$$

Now, from Proposition 8.16 we know that $\lfloor-\rfloor$ preserves surjective open maps, and so does $\operatorname{Lan}_{\mathrm{yP}} F$ by Corollary 3.3. Hence their composition, and so $G$, preserves surjective open maps, and consequently open map bisimulation.

Via the reflection Cocont $\stackrel{\longleftarrow}{\longleftarrow}$ Conn, the category Conn inherits a monoidal closed structure from Cocont, and is sufficiently rich in operations to give semantics to a broad spectrum of process languages, including those with a form of linear process passing. Affine HOPLA is such a linear process passing language, introduced in [39, 40]; its operations, definable within Conn preserve open map bisimulation leading automatically to congruence results $[52,11]$. The category Conn also supports a trace operation associated with a feedback loop in nondeterministic dataflow [24].

\section{Pseudo comonads via families}

\subsection{Motivation}

According to the discipline of linear logic, nonlinear maps from $\mathbb{P}$ to $\mathbb{Q}$ are introduced as linear maps from $! \mathbb{P}$ to $\mathbb{Q}$ - the exponential! applied to $\mathbb{P}$ allows arguments from $\mathbb{P}$ to be copied or discarded freely. We have interpreted $! \mathbb{P}$ as the finite-colimit completion of $\mathbb{P}$. With this understanding of $! \mathbb{P}$, linear maps $! \mathbb{P} \rightarrow \mathbb{Q}$ correspond, to within isomorphism, to filtered colimit preserving functors from $\widehat{\mathbb{P}}$ to $\widehat{\mathbb{Q}}$. But, unfortunately, continuous functors from $\widehat{\mathbb{P}}$ to $\widehat{\mathbb{Q}}$ need not preserve bisimulation. This raises the question of whether other choices of exponential fit better with open maps and bisimulation.

Observe the hopeful sign that maps which are not linear may still preserve bisimulation. For example, a functor yielding a presheaf $H(X, Y)$, for presheaves $X$ and $Y$ over $\mathbb{P}$, which is "bilinear" in the sense that it preserves colimits in each argument separately, when diagonalised to the functor giving $H(X, X)$ for $X$ in $\widehat{\mathbb{P}}$, will still preserve open maps and bisimulation. A well-known example of a bilinear functor is the product operation on presheaves [27]. For essentially the same reason the tensor operation in Prof is bilinear and preserves open maps. 
Bear in mind the intuition that objects of $\mathbb{P}$ correspond to the shapes of computation path a process, represented as a presheaf in $\widehat{\mathbb{P}}$, might perform. An object of $! \mathbb{P}$ should represent a computation path of an assembly of processes each with computation-path shapes in $\mathbb{P}$ - the assembly of processes can then be the collection of copies of a process, possibly at different states. If we take $\mathbb{P}$ to be the finite colimit completion of $\mathbb{P}$, an object of $! \mathbb{P}$ as a finite colimit would express how paths coincide initially and then branch. To understand this object as a computation path of an assembly of processes, we can view the assembly of processes as not fixed once and for all. Rather the assembly grows as further copies are invoked, and these copies can be made of a processes after they have run for a while. The copies can then themselves be run and the resulting processes copied. In this way, by keeping track of the origins of copies, we can account for the identifications of sub-paths.

This intuition suggests exploring other less liberal ways of copying, without, for example, being able to copy after some initial run. If we are to index different copies to distinguish them we are led to consider indexed families of objects in a category.

\subsection{Indexed families}

Definition 9.1 Let $\mathbb{U}$ be a subcategory of Set. Let $\mathcal{A} \in \mathbf{C A T}$. Define $\mathcal{F}_{\mathbb{U}}(\mathcal{A})$ to be the category of $\mathbb{U}$-families which consists of

- objects $\left\langle A_{i}\right\rangle_{i \in I}$ where $I \in|\mathbb{U}|$ and $A_{i} \in|\mathcal{A}|$, for all $i \in I$.

- $\operatorname{arrows}(f, e):\left\langle A_{i}\right\rangle_{i \in I} \rightarrow\left\langle A_{j}^{\prime}\right\rangle_{j \in J}$ where $f: I \rightarrow J$ in $\mathbb{U}$ and $e=\left\langle e_{i}\right\rangle_{i \in I}$ such that $e_{i}: A_{i} \rightarrow A_{f(i)}^{\prime}$, for all $i \in I$.

The operation $\mathcal{F}_{\mathbb{U}}$ extends to a 2-functor on $\mathbf{C A T}$. Letting $F: \mathcal{A} \rightarrow \mathcal{B}$, the functor $\mathcal{F}_{\mathbb{U}}(F): \mathcal{F}_{\mathbb{U}}(\mathcal{A}) \rightarrow \mathcal{F}_{\mathbb{U}}(\mathcal{B})$ takes

$$
\left(f,\left\langle e_{i}\right\rangle_{i \in I}\right):\left\langle A_{i}\right\rangle_{i \in I} \rightarrow\left\langle A_{j}^{\prime}\right\rangle_{j \in J}
$$

to

$$
\left(f,\left\langle F e_{i}\right\rangle i \in I\right):\left\langle F A_{i}\right\rangle_{i \in I} \rightarrow\left\langle F A_{j}^{\prime}\right\rangle_{j \in J} .
$$

For $\varphi: F \Rightarrow G$, define $\mathcal{F}_{\mathbb{U}}(\varphi): \mathcal{F}_{\mathbb{U}}(F) \Rightarrow \mathcal{F}_{\mathbb{U}}(G)$ as

$$
\mathcal{F}_{\mathbb{U}}(\varphi)_{\left\langle A_{i}\right\rangle_{i \in I}}=\left(1_{I},\left\langle\varphi_{A_{i}}\right\rangle_{i \in I}\right):\left\langle F A_{i}\right\rangle_{i \in I} \longrightarrow\left\langle G A_{i}\right\rangle_{i \in I}
$$

It is easy to see that

$$
\mathcal{F}_{\mathbb{U}}(\mathcal{A})\left(\left\langle A_{j}\right\rangle_{j \in J},\left\langle A_{i}^{\prime}\right\rangle_{i \in I}\right) \cong \sum_{f \in \mathbb{U}(J, I)} \prod_{j \in J} \mathcal{A}\left(A_{j}, A_{f(j)}^{\prime}\right) .
$$

Under sufficient conditions, that $\mathbb{U}$ is small, has singletons and dependent sums, we can obtain a 2-monad on CAT.

Definition 9.2 A dependent sum for $\mathbb{U}$ is a functor $\sum: \mathcal{F}_{\mathbb{U}}(\mathbb{U}) \rightarrow \mathbb{U}$ such that

- on objects $\left\langle J_{i}\right\rangle_{i \in I}$ of $\mathcal{F}_{\mathbb{U}}(\mathbb{U})$, the object $\sum\left(\left\langle J_{i}\right\rangle_{i \in I}\right)$ is a sum (disjoint union) of sets $\sum_{i \in I} J_{i}$; write $[i, j]$ for the $i$-th injection of $j$ into the sum.

- on arrows $(f, g):\left\langle J_{i}\right\rangle_{i \in I} \rightarrow\left\langle J_{i^{\prime}}^{\prime}\right\rangle_{i^{\prime} \in I^{\prime}}$ of $\mathcal{F}_{\mathbb{U}}(\mathbb{U})$; so $f: I \rightarrow I^{\prime}$ and $g=\left\langle g_{i}\right\rangle_{i \in I}$ is a family of maps $g_{i}: J_{i} \rightarrow J_{f(i)}^{\prime}$ in $\mathbb{U}$,

$$
\sum(f, g): \sum_{i \in I} J_{i} \rightarrow \sum_{i^{\prime} \in I^{\prime}} J_{i^{\prime}}^{\prime} ;[i, j] \mapsto\left[f(i), g_{i}(j)\right] .
$$


For $\mathbb{U}$ with a singleton, we can define the functor $\eta_{\mathcal{A}}: \mathcal{A} \rightarrow \mathcal{F}_{\mathbb{U}}(\mathcal{A})$ which sends $A$ in $\mathcal{A}$ to the singleton family with $A$ as its single component. For $\mathbb{U}$ with dependent sum, we can define the functor $\mu_{\mathcal{A}}: \mathcal{F}_{\mathbb{U}} \mathcal{F}_{\mathbb{U}}(\mathcal{A}) \rightarrow \mathcal{F}_{\mathbb{U}}(\mathcal{A})$ which takes a family of families $\left\langle\left\langle A_{i, j}\right\rangle_{j \in J_{i}}\right\rangle_{i \in I}$ to the family $\left\langle A_{i, j}\right\rangle_{[i, j] \in \sum_{i \in I} J_{i}}$. Under the conditions that $\mathbb{U}$ has a singleton set $\{*\}$ as object, and a dependent sum, $\mathcal{F}_{\mathbb{U}}$ becomes a 2 -monad on CAT; its unit $\eta$ has components $\eta_{\mathcal{A}}$ and its multiplication components $\mu_{\mathcal{A}}$.

\subsection{Pseudo-comonads on Prof}

We will think of profunctors $\mathcal{F}_{\mathbb{U}}(\mathbb{P}) \rightarrow \rightarrow \mathbb{Q}$ as generalised forms of polynomials. ${ }^{5}$ Consider the category of "polynomials" $\operatorname{Prof}\left(\mathcal{F}_{\mathbb{U}}(\mathbb{P}), \mathbb{Q}\right)$ from $\mathbb{P}$ to $\mathbb{Q}$; the category is clearly isomorphic to the presheaf category $\left(\mathcal{F}_{\mathbb{U}} \widehat{(\mathbb{P}))^{\mathrm{op}}} \times \mathbb{Q}\right.$, so has open maps, and the functor category $\left[\mathcal{F}_{\mathbb{U}}(\mathbb{P}), \widehat{\mathbb{Q}}\right]$. Under sufficient conditions, that $\mathbb{U}$ is small, has a singleton and dependent sums, we can compose polynomials in the manner of the co-Kleisli construction. To do this we use a distributive law converting a family of presheaves into a presheaf over families of paths.

The following distributive law is used to turn $\mathcal{F}_{\mathbb{U}}$ into a pseudo functor on Prof. For a small category $\mathbb{Q}$,

$$
d_{\mathbb{Q}}=\left(\mathcal{F}_{\mathbb{U}} \mathrm{y}_{\mathbb{Q}}\right)^{*}: \mathcal{F}_{\mathbb{U}}(\widehat{\mathbb{Q}}) \rightarrow \widehat{\mathcal{F}_{\mathbb{U}}(\mathbb{Q})}
$$

Recall from the Appendix, A.4.3, that this means that

$$
d_{\mathbb{Q}}\left(\left\langle X_{i}\right\rangle_{i \in I}\right)=\mathcal{F}_{\mathbb{U}}(\widehat{\mathbb{Q}})\left(\mathcal{F}_{\mathbb{U}}\left(\mathrm{y}_{\mathbb{Q}}\right)(-),\left\langle X_{i}\right\rangle_{i \in I}\right)
$$

for $\left\langle X_{i}\right\rangle_{i \in I}$ in $\mathcal{F}_{\mathbb{U}}(\widehat{\mathbb{Q}})$. It thus acts so

$$
d_{\mathbb{Q}}\left(\left\langle X_{i}\right\rangle_{i \in I}\right)\left\langle q_{j}\right\rangle_{j \in J} \cong \sum_{f \in \mathbb{U}(J, I)} \prod_{j \in J} X_{f(j)}\left(q_{j}\right),
$$

for $\left\langle X_{i}\right\rangle_{i \in I}$ in $\mathcal{F}_{\mathbb{U}}(\widehat{\mathbb{Q}})$ and $\left\langle q_{j}\right\rangle_{j \in J}$ in $\mathcal{F}_{\mathbb{U}}(\mathbb{Q})$ - as is easy to show.

With the help of the distributive law we can define a pseudo-endofunctor on Prof: on objects it acts as $\mathcal{F}_{\mathbb{U}}$, and sends an arrow $F: \mathbb{P} \mapsto \mathbb{Q}$ to $\overline{d_{\mathbb{Q}} \circ\left(\mathcal{F}_{\mathbb{U}} \bar{F}\right)}$ : $\mathcal{F}_{\mathbb{U}}(\mathbb{P}) \longrightarrow \mathcal{F}_{\mathbb{U}}(\mathbb{Q})$, and a 2-cell $\alpha: F \Rightarrow G$ to $d_{\mathbb{Q}}\left(\mathcal{F}_{\mathbb{U}} \alpha\right)$.

The pseudo-functor has a counit $\varepsilon$ and comultiplication $\delta$ with components

$$
\begin{aligned}
& \varepsilon_{\mathbb{P}}=\overline{\eta_{\mathbb{P}}^{*} \circ \mathrm{y}_{\mathcal{F}_{\mathbb{U}}(\mathbb{P})}}: \mathcal{F}_{\mathbb{U}}(\mathbb{P}) \rightarrow \mathbb{P} \\
& \delta_{\mathbb{P}}=\overline{\mu_{\mathbb{P}}^{*} \circ \mathrm{y}_{\mathcal{F}_{\mathbb{U}}(\mathbb{P})}}: \mathcal{F}_{\mathbb{U}}(\mathbb{P}) \rightarrow \mathcal{F}_{\mathbb{U}} \mathcal{F}_{\mathbb{U}}(\mathbb{P}) .
\end{aligned}
$$

With suitable coherence modifications this turns $\mathcal{F}_{\mathbb{U}}$ into a pseudo comonad.

Notation: From now on we will use the $\mathcal{F}_{\mathbb{U}}$ for the pseudo-functor on Prof.

\footnotetext{
${ }^{5}$ This view is amplified in $[39,53]$. For now, note that special profunctors of this form, viz., $\mathcal{F}_{\mathbb{B}}(\mathbb{1}) \rightarrow \longrightarrow \mathbb{1}$ where $\mathbb{B}$ is the category of finite sets and bijections, are used in Joyal's theory of species [3]. A profunctor $\mathcal{F}_{\mathbb{B}}(\mathbb{1}) \rightarrow \mathbb{1}$ corresponds to a functor $F: \mathbb{B} \rightarrow$ Set; such a functor in turn corresponds to an analytic functor from Set to Set, taking a set $X$ to $\int{ }^{n \in \mathbb{B}} F n \cdot X^{n}$. See Example 9.9.
} 
Its convenient to write a polynomial $F: \mathcal{F}_{\mathbb{U}}(\mathbb{P})-\rightarrow \rightarrow \mathbb{Q}$, an arrow in Prof as $F$ : $\mathbb{P} \rightarrow \mathbb{U} \mathbb{Q}$. The composition of two such polynomials $F: \mathbb{P} \rightarrow \mathbb{U} \mathbb{Q}$ and $G: \mathbb{Q} \rightarrow \mathbb{U}$ is given, as in the construction of a co-Kleisli category, by the composition

$$
\mathcal{F}_{\mathbb{U}}(\mathbb{P}) \stackrel{\delta_{\mathbb{P}}}{\rightarrow} \mathcal{F}_{\mathbb{U}} \mathcal{F}_{\mathbb{U}}(\mathbb{P}) \stackrel{\mathcal{F}_{\mathbb{U}}(F)}{+\rightarrow} \mathcal{F}_{\mathbb{U}}(\mathbb{Q}) \stackrel{G}{\rightarrow} \mathcal{F}_{\mathbb{U}}(\mathbb{R})
$$

Assume that $\mathbb{U}$, the subcategory of Set, contains the empty set; then $\mathcal{F}_{\mathbb{U}}(\mathbb{O})$, families of the empty category, will be isomorphic to the category $\mathbb{1}$ consisting of a single object and its identity arrow. A $\mathbb{U}$-polynomial $F: \mathbb{P} \rightarrow \mathbb{U} \mathbb{Q}$ gives rise to a functor $F^{\dagger}: \widehat{\mathbb{P}} \rightarrow \widehat{\mathbb{Q}}$ in the following way. Viewing a presheaf $X$ in $\widehat{\mathbb{P}}$ as a profunctor $\mathbb{1} \rightarrow \mathbb{P}$ we can also see it as an arrow $\mathbb{O} \stackrel{X}{\longrightarrow} \mathbb{U} \mathbb{P}$. We define $F^{\dagger}(X)$ as the presheaf obtained by the composition of polynomials

$$
\mathbb{O} \stackrel{X}{\longrightarrow} \mathbb{U} \stackrel{F}{\longrightarrow} \mathbb{U} \mathbb{Q} \text {. }
$$

The result $F^{\dagger}(X)$ is the application of the polynomial $F$ to the presheaf $X$. By simplification of the associated coend expression, the functor $F^{\dagger}$ obtained in this way can be shown to coincide with the left Kan extension $\operatorname{Lan}_{J_{\mathbb{J}}} \bar{F}$, where $J_{\mathbb{U}}: \mathcal{F}_{\mathbb{U}}(\mathbb{P}) \rightarrow \widehat{\mathbb{P}}$ is the functor given on objects by

$$
J_{\mathbb{U}}\left(\left\langle P_{i}\right\rangle_{i \in I}\right)=\sum_{i \in I} \mathrm{y}_{\mathbb{P}}\left(P_{i}\right)
$$

and on arrows $(f, e):\left\langle P_{i}\right\rangle_{i \in I} \rightarrow\left\langle P_{j}^{\prime}\right\rangle_{j \in J}$ by the mediating arrow

$$
J_{\mathbb{U}}(f, e)=\left[i n_{f(i)}^{\prime} \circ \mathrm{y}_{\mathbb{P}}\left(e_{i}\right)\right]_{i \in I}: \sum_{i \in I} \mathrm{y}_{\mathbb{P}}\left(P_{i}\right) \rightarrow \sum_{j \in J} \mathrm{y}_{\mathbb{P}}\left(P_{j}^{\prime}\right),
$$

where $i n_{j}^{\prime}$ are the injections $\mathrm{y}_{\mathbb{P}}\left(\mathbb{P}_{j}^{\prime}\right) \rightarrow \sum_{j \in J} \mathrm{y}_{\mathbb{P}}\left(P_{j}^{\prime}\right)$ :

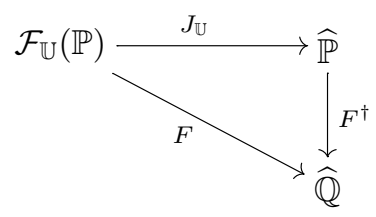

There is the question as to whether the functor $F^{\dagger}: \widehat{\mathbb{P}} \rightarrow \widehat{\mathbb{Q}}$ determines, to within isomorphism, the polynomial $F: \mathbb{P} \rightarrow \mathbb{U} \mathbb{Q}$ from which it is derived. This property holds for interesting special cases: when for instance $J_{\mathbb{U}}$ is full and faithful; and the case of analytic functors [3] obtained when $\mathbb{U}$ consists of finite sets and bijections and $\mathbb{P}$ and $\mathbb{Q}$ are both $\mathbb{1}$-see 9.9 . When polynomials correspond to functors between presheaves we have the simplification of being able to work with 2-category based on the composition of functors rather than a bicategory of polynomials. For $\mathbb{U}$ in general, non-isomorphic polynomials can give rise to isomorphic functors between presheaf categories.

\subsection{On preservation of bisimulation}

For simplicity we only consider preservation of bisimulation by functors $F^{\dagger}$ for polynomials $F: \mathcal{F}_{\mathbb{U}}(\mathbb{P}) \rightarrow+\rightarrow \mathbb{Q}$ (though corresponding results hold for the composition of polynomials). The functor $F^{\dagger}$ coincides with $\operatorname{Lan}_{J_{\mathbb{U}}} \bar{F}$. By Proposition A.14,

$$
F^{\dagger} \cong\left(\operatorname{Lan}_{\mathcal{F}_{\mathcal{F}_{\mathbb{U}}(\mathbb{P})}}(\bar{F})\right) \circ J_{\mathbb{U}}^{*}
$$


This factorisation suggests that we should examine how

$$
J_{\mathbb{U}}^{*}: \widehat{\mathbb{P}} \rightarrow \widehat{\mathcal{F}_{\mathbb{U}}(\mathbb{P})}
$$

preserves bisimulation. For this, it is important to remark that

$$
J_{\mathbb{U}}^{*} X\left(\left\langle P_{j}\right\rangle_{j \in J}\right) \cong \prod_{j \in J} X\left(P_{j}\right),
$$

natural in $X$ and $\left\langle P_{j}\right\rangle_{j \in J}$. This follows from the definition of $J_{\mathbb{U}}^{*}$, as $J_{\mathbb{U}}^{*} X=\widehat{\mathbb{P}}\left(J_{\mathbb{U}}(-), X\right)$, natural in $X$, and the chain of isomorphisms

$$
J_{\mathbb{U}}^{*} X\left(\left\langle P_{j}\right\rangle_{j \in J}\right) \cong \widehat{\mathbb{P}}\left(\sum_{j \in J} \mathrm{y} \mathbb{P}\left(P_{j}\right), X\right) \cong \prod_{j \in J} \widehat{\mathbb{P}}\left(\mathrm{y}_{\mathbb{P}}\left(P_{j}\right), X\right) \cong \prod_{j \in J} X\left(P_{j}\right),
$$

natural in $X$ and $\left\langle P_{j}\right\rangle_{j \in J}$.

First notice that $J_{\mathbb{U}}^{*}$ preserves surjectivity:

Proposition 9.3 Suppose that $h: X \rightarrow Y$ is a surjective map in $\widehat{\mathbb{P}}$. Then, $J_{\mathbb{U}}^{*} h$ is a surjective map in $\widehat{\mathcal{F}_{\mathbb{U}}(\mathbb{P})}$.

Proof: As remarked above,

$$
J_{\mathbb{U}}^{*} X\left(\left\langle P_{j}\right\rangle_{j \in J}\right) \cong \prod_{j \in J} X\left(P_{j}\right),
$$

natural in $X$ and $\left\langle P_{j}\right\rangle_{j \in J}$. In particular, we have the naturality square

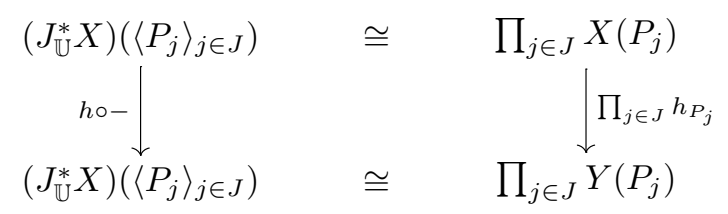

associated with $h: X \rightarrow Y$. Clearly if $h$ is surjective, then each function $h_{P_{j}}$ is surjective, ensuring that the function $\left(J_{\mathbb{U}}^{*} h\right)_{\left\langle P_{j}\right\rangle_{j \in J}}=h \circ-$ is surjective too.

Consider the factorisation $(\dagger)$ of $F^{\dagger}$. By Lemma 2.6 the functor $J_{\mathbb{U}}^{*}$ sends $J_{\mathbb{U}}$-open maps to $\mathrm{y}_{\mathcal{F}_{\mathbb{U}}(\mathbb{P})}$-open maps, which are then sent by the left Kan extension $\operatorname{Lan}_{\mathcal{J}_{\mathcal{F}_{\mathbb{U}}(\mathbb{P})}} \bar{F}$ to y $\mathbb{Q}^{-o p e n ~ m a p s . ~ F u r t h e r m o r e, ~ b o t h ~} J_{\mathbb{U}}^{*}$ and $\operatorname{Lan}_{\mathcal{y}_{\mathcal{U}}(\mathbb{P})}(\bar{F})$ preserve surjectivity. The question of preservation of bisimulation hinges on the nature of $J_{\mathbb{U}}$-open maps. This depends on the choice of $\mathbb{U}$. The next proposition deals with two important general cases.

Proposition 9.4 Let $\mathbb{U}$ be a subcategory of finite sets and functions, which has a singleton.

(i) Suppose $\mathbb{U}$ contains a map $2 \rightarrow 1$ from a set 2 with two distinct elements to a singleton 1 . Then, any surjective $J_{\mathbb{U}}$-open map in $\widehat{\mathbb{P}}$ is an isomorphism.

(ii) Suppose that all maps in $\mathbb{U}$ are injections. A surjective map in $\widehat{\mathbb{P}}$ is $J_{\mathbb{U}}$-open iff it is $\mathrm{y}_{\mathbb{P}}$-open. 


\section{Proof:}

(i) By copying the proof of Proposition 7.11, which, as stated in its acompanying remark, applies quite generally.

(ii) Because $\mathbb{U}$ has singletons, $\mathrm{y}_{\mathbb{P}} \cong J_{\mathbb{U}} \circ \eta_{\mathbb{P}}$ - the Yoneda embedding factors through

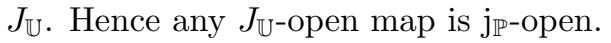

Conversely, suppose that $h$ is surjective and open. We show that $J_{\mathbb{U}}^{*} h$ is open in $\widehat{\mathcal{F}_{\mathbb{U}}(\mathbb{P})}$ - by Lemma 2.6 this is equivalent to $h$ being $J_{\mathbb{U}}$-open. As remarked earlier,

$$
J_{\mathbb{U}}^{*} X\left(\left\langle P_{i}\right\rangle_{i \in I}\right) \cong \prod_{i \in I} X\left(P_{i}\right)
$$

natural in $X$ and $\left\langle P_{i}\right\rangle_{i \in I}$

Consider the naturality square

$$
\begin{aligned}
& \prod_{i \in I} X\left(P_{i}\right) \stackrel{\prod_{i \in I} X m_{i}}{\longleftarrow} \prod_{j \in J} X\left(Q_{j}\right) \\
& \prod_{i \in I} h_{P_{i}} \downarrow \downarrow \prod_{j \in J} h_{Q_{j}} \\
& \prod_{i \in I} Y\left(P_{i}\right) \underset{\prod_{i \in I} Y m_{i}}{\downarrow} \prod_{j \in J} Y\left(Q_{j}\right)
\end{aligned}
$$

associated with the arrow

$$
\left(f,\left\langle m_{i}\right\rangle_{i \in I}\right):\left\langle P_{i}\right\rangle_{i \in I} \rightarrow\left\langle Q_{j}\right\rangle_{j \in J}
$$

in $\mathcal{F}_{\mathbb{U}}(\mathbb{P})$. Note that we have, for instance, written $\prod_{i \in I} X m_{i}: \prod_{j \in J} X\left(Q_{j}\right) \rightarrow$ $\prod_{i \in I} Y\left(P_{i}\right)$ for the map taking $\left\langle x_{j}^{\prime}\right\rangle_{j \in J}$ to $\left\langle X m_{i}\left(x_{f(i)}^{\prime}\right)\right\rangle_{i \in I}$.

We must show that the square is a quasi-pullback. To this end, suppose that

$$
\left(\prod_{i \in I} h_{P_{i}}\right)\left(\left\langle x_{i}\right\rangle_{i \in I}\right)=\left(\prod_{i \in I} Y m_{i}\right)\left(\left\langle y_{j}^{\prime}\right\rangle_{j \in J}\right)=\left\langle y_{i}\right\rangle_{i \in I} .
$$

We now describe how to produce the components of a tuple $x^{\prime}=\left\langle x_{j}^{\prime}\right\rangle_{j \in J} \in \prod_{j \in J} X\left(Q_{j}\right)$ such that

$$
\left(\prod_{i \in I} X m_{i}\right)\left(x^{\prime}\right)=\left\langle x_{i}\right\rangle_{i \in I} \text { and }\left(\prod_{j \in J} h_{Q_{j}}\right)\left(x^{\prime}\right)=\left\langle y_{j}^{\prime}\right\rangle_{j \in J} .
$$

For each $j=f(i) \in J$, for some $i \in I$, the square

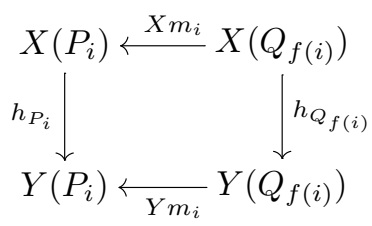

is a quasi-pullback, in which

$$
h_{P_{i}}\left(x_{i}\right)=\left(Y m_{i}\right)\left(y_{f(i)}^{\prime}\right)=y_{i} .
$$

Hence there exists some $x_{f(i)}^{\prime}$ such that

$$
X m_{i}\left(x_{f(i)}^{\prime}\right)=x_{i} \text { and } h_{Q_{f(i)}}\left(x_{f(i)}^{\prime}\right)=y_{f(i)}^{\prime} .
$$

For each $j \in J$ not in the range of $f$, because $h_{Q_{j}}$ is surjective there is $x_{j}^{\prime}$ such that $h_{Q_{j}}\left(x_{j}^{\prime}\right)=y_{j}^{\prime}$.

Taking $x^{\prime}=\left\langle x_{j}^{\prime}\right\rangle_{j \in J}$ we fulfill the quasi-pullback condition for $J_{\mathbb{U}}^{*} h$ to be open. 
If the empty set is an object in $\mathbb{U}$ it need not be initial, for example, if the maps of $\mathbb{U}$ are bijections. In general $J_{\mathbb{U}}$-open maps need not be surjective. However:

Proposition 9.5 If $\mathbb{U}$ includes the function $\emptyset \rightarrow 1$ from the empty set to a singleton, then $J_{\mathbb{U}}$-open maps are surjective open.

Proof: In this case the functor $j_{\mathbb{P}_{\perp}}: \mathbb{P}_{\perp} \rightarrow \widehat{\mathbb{P}}$ factors through $J_{\mathbb{U}}$ via the functor $\mathbb{P}_{\perp} \rightarrow \mathcal{F}_{\mathbb{U}}(\mathbb{P})$ taking $\perp$ to the empty family and objects of $\mathbb{P}$ to their corresponding singleton families.

We consider different examples of $\mathbb{U}$ and the families and polynomials and properties they give rise to.

Example 9.6 Consider the subcategory of sets $\Omega$ which consists of objects subsets $\underline{n}=\{1, \cdots, n\}$, empty when $n=0$, of positive natural numbers with identities as the only maps. Then, $\mathcal{F}_{\Omega}(\mathbb{P})$ is isomorphic to

$$
\mathbb{1}+\mathbb{P}+\mathbb{P}^{2}+\mathbb{P}^{3}+\cdots+\mathbb{P}^{k}+\cdots .
$$

Here the superscripts abbreviate repeated applications of tensor in Prof, so $\mathbb{P}^{k}$ is the product of $k$ copies of the category $\mathbb{P}$ - in particular, $\mathbb{1}$ is the category consisting solely of the empty tuple.

The category $\mathbb{U}$ has a singleton, viz. $\underline{1}=\{1\}$. Its dependent sum is given by:

$$
\sum_{i \in \underline{k}} \underline{j_{i}}=\underline{j_{1}+\cdots+j_{k}} .
$$

Clearly all the maps of $\Omega$ are injections so, by Proposition 9.4(ii), maps which are $J_{\Omega}$-open are surjective open. It follows that application (and, in fact, also composition) of $\Omega$-polynomials preserves surjective open maps, so bisimulation.

However, there is no reasonable sense in which taking $\Omega$-polynomials as maps yields a cartesian-closed bicategory. It easy to see that there is an isomorphism of categories

$$
\operatorname{Prof}\left(\mathcal{F}_{\Omega}(\mathbb{R}), \mathbb{P} \& \mathbb{Q}\right) \cong \operatorname{Prof}\left(\mathcal{F}_{\Omega}(\mathbb{R}), \mathbb{P}\right) \times \operatorname{Prof}\left(\mathcal{F}_{\Omega}(\mathbb{R}), \mathbb{Q}\right)
$$

in fact pseudo-natural in $\mathbb{R}$, showing the sense in which $\mathbb{P} \& \mathbb{Q}$, given by juxtaposition, remains a product with polynomials as maps. There is also clearly an isomorphism of functor categories

$$
\operatorname{Prof}\left(\mathcal{F}_{\Omega}(\mathbb{P}) \times \mathcal{F}_{\Omega}(\mathbb{Q}), \mathbb{R}\right) \cong \operatorname{Prof}\left(\mathcal{F}_{\Omega}(\mathbb{P}),\left(\left(\mathcal{F}_{\Omega}(\mathbb{Q})\right)^{\mathrm{op}} \times \mathbb{R}\right)\right)
$$

But, in general, $\mathcal{F}_{\Omega}(\mathbb{P} \& \mathbb{Q})$ and $\mathcal{F}_{\Omega}(\mathbb{P}) \times \mathcal{F}_{\Omega}(\mathbb{Q})$ are not isomorphic (the analogue of the Seely condition $[47]$ is not met), so that $\left(\mathcal{F}_{\Omega}(\mathbb{Q})\right)^{\text {op }} \times \mathbb{R}$ is not a function space for the polynomials with respect to $-\&-$. (This example is dealt with in more detail in $[39,53]$.)

Example 9.7 Now consider the full subcategory of sets $\mathbb{F}$ consisting of all finite sets with functions as arrows. (Alternatively we can work with the equivalent category in which the objects are natural numbers understood as sets, as in $\Omega$ above, but this time allowing all functions as maps.) In this case $\mathcal{F}_{\mathbb{F}}(\mathbb{P})$ is the finite coproduct completion of a small category $\mathbb{P}$ (a construction dual to the categorical powerdomain [32, 1]). 
Clearly $\mathbb{F}$ has singletons. It has a dependent sum given by disjoint union.

There is an isomorphism

$$
\mathcal{F}_{\mathbb{F}}(\mathbb{P}) \otimes \mathcal{F}_{\mathbb{F}}(\mathbb{Q}) \cong \mathcal{F}_{\mathbb{F}}(\mathbb{P} \& \mathbb{Q})
$$

expressing how a family in $\mathcal{F}_{\mathbb{F}}(\mathbb{P} \& \mathbb{Q})$ can be broken down into a pair of families, one component from $\mathcal{F}_{\mathbb{F}}(\mathbb{P})$ and the other from $\mathcal{F}_{\mathbb{F}}(\mathbb{Q})$. So the analogue of the Seely condition is met, and $\left(\mathcal{F}_{\mathbb{F}}(\mathbb{Q})\right)^{\text {op }} \times \mathbb{R}$ is a reasonable function space.

But, by Proposition $9.4(\mathrm{i})$, in this case $J_{\mathbb{F}}$-bisimulation is degenerate and coincides with isomorphism. Application (and composition) of $\mathbb{F}$-polynomials does not in general preserve open map bisimulation. Because the functors $\mathbb{J}_{\mathbb{F}}$ are full and faithful, $\mathbb{F}$-polynomials correspond to within isomorphism to special functors between presheaf categories (under suitable conditions, they are exact functors [10]).

Example 9.8 The category II consists of finite sets and injections. (Alternatively we can work with the equivalent category with objects natural numbers understood as sets with injections.)

There is an isomorphism

$$
\mathcal{F}_{\mathbb{I}}(\mathbb{P}) \otimes \mathcal{F}_{\mathbb{I}}(\mathbb{Q}) \cong \mathcal{F}_{\mathbb{I}}(\mathbb{P} \& \mathbb{Q})
$$

expressing how a family in $\mathcal{F}_{\mathbb{I}}(\mathbb{P} \& \mathbb{Q})$ can be broken down into a pair of families - the Seely condition. This ensures an isomorphism of functor categories

$$
\operatorname{Prof}\left(\mathcal{F}_{\mathbb{I}}(\mathbb{P} \& \mathbb{Q}), \mathbb{R}\right) \cong \operatorname{Prof}\left(\mathcal{F}_{\mathbb{I}}(\mathbb{P}),\left(\left(\mathcal{F}_{\mathbb{I}}(\mathbb{Q})\right)^{\mathrm{op}} \times \mathbb{R}\right)\right)
$$

the sense in which $\left(\mathcal{F}_{\mathbb{I}}(\mathbb{Q})\right)^{\text {op }} \times \mathbb{R}$ is a function space when maps are $\mathbb{I}$-polynomials.

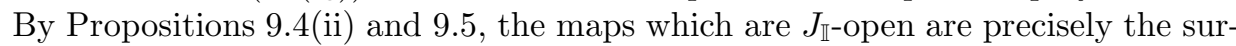
jective open maps, so that application (and, in fact, also composition) of I-polynomials preserves surjective open maps and bisimulation.

It is possible for two non-isomorphic $\mathbb{I}$-polynomials $F, G: \mathcal{F}_{\mathbb{I}}(\mathbb{1}) \rightarrow \mathbb{1}$ to give rise to isomorphic functors $F^{\dagger} \cong G^{\dagger}:$ Set $\rightarrow$ Set. (Our counterexample relies on one of the functors not preserving pullbacks.)

$\mathcal{F}_{\mathbb{I}}$ seems a sensible choice of exponential. With $\mathcal{F}_{\mathbb{I}}$ processes may be copied some arbitrary and extensible number of times, the copies being assembled as tuples with shape an object in $\mathbb{I}$.

If we restrict families to the full subcategory of $\mathbb{I}_{0}$ of $\mathbb{I}$ consisting of just two objects, the empty and singleton sets, we obtain $\mathcal{F}_{\mathbb{I}_{0}}(\mathbb{P}) \cong \mathbb{P}_{\perp}$. With $\mathbb{I}_{0}$-polynomials (a form of "affine" polynomials) we obtain a biequivalence with Conn:

$$
\operatorname{Prof}\left(\mathcal{F}_{\mathbb{I}_{0}}(\mathbb{P}), \mathbb{Q}\right) \simeq \operatorname{Conn}(\mathbb{P}, \mathbb{Q})
$$

Example 9.9 The category $\mathbb{B}$ consists of finite sets and bijections. (Alternatively we get a category equivalent to $\mathbb{B}$ by taking objects the natural numbers understood as sets with permutations as maps.)

We have the Seely condition

$$
\mathcal{F}_{\mathbb{B}}(\mathbb{P}) \otimes \mathcal{F}_{\mathbb{B}}(\mathbb{Q}) \cong \mathcal{F}_{\mathbb{B}}(\mathbb{P} \& \mathbb{Q})
$$

and accordingly a function space $\left(\mathcal{F}_{\mathbb{B}}(\mathbb{Q})\right)^{\text {op }} \times \mathbb{R}$. 
By Proposition 9.4(ii), maps which are surjective $J_{\mathbb{B}}$-open are surjective open, so that application (and, in fact, also composition) of $\mathbb{B}$-polynomials preserves surjective open maps and bisimulation.

With $\mathcal{F}_{\mathbb{B}}$ as the choice of exponential, processes may be copied some arbitrary but non-extensible number of times. We obtain another form of "affine" polynomials if we restrict families to the full subcategory of $\mathbb{B}_{0}$ of $\mathbb{B}$ consisting of just two objects, the empty and singleton sets; in this case, $\mathcal{F}_{\mathbb{B}_{0}}(\mathbb{P}) \cong \mathbb{P}+\mathbb{1}$.

In general we can specialise $\mathbb{U}$-polynomials to polynomials $F: \mathbb{1} \rightarrow \mathbb{U}$. As $\mathcal{F}_{U}(\mathbb{1}) \cong \mathbb{U}$, such $\mathbb{U}$-polynomials are functors $F: \mathbb{U} \rightarrow$ Set. In particular, special $\mathbb{B}$-polynomials, functors $F: \mathbb{B} \rightarrow$ Set correspond up to isomorphism to analytic functors $F^{\dagger}:$ Set $\rightarrow$ Set $[3]$.

\section{Conclusions}

This paper lays down the basic mathematics which underlies a theory of processes at the level of intricacy found in concurrent computation. We have found the mathematics essential in developing a domain theory for concurrency.

The mathematics has a life of its own, which is only patchily covered and understood in terms of existing process languages and their operational semantics. There have been successes in applying the mathematics, in connecting with process languages and operational semantics [39, 40], the semantics of nondeterministic dataflow [24], independence/causal models [23, 38], fairness [22], pi-Calculus and name generation for higher order processes [13, 57], and weak bisimulation [17]. These are all examples of how we can bring categorical reasoning to bear on issues of concurrent computation. (Much of this work is summarised along with the present limitations in [40].) But there is still some way to go in making that mathematics operational. For example, a full operational understanding of open map bisimulation for higher order processes would seem to require a syntax and operational reading of the duality between input and output given by $(-)^{\perp}$ in the bicategory of profunctors.

One way forward is to build operational semantics from the presheaf semantics; a guiding principle has been that elements of presheaves should correspond to derivations in an operational semantics. Another is via representations of presheaf denotations in terms of more traditional process models such as event structures; these can give a more detailed understanding of elements of presheaves (and so derivations in an operational semantics) as configurations of an event structure.

Such work is likely to take us to refinements of profunctors and open map bisimulation, and to other (bi)categories. But the results of this paper make, we believe, a strong case that the links between nondeterministic processes and profunctors, operations on processes and categorical constructions, open maps and bisimulation, are truly fundamental.

\section{Acknowledgements}

Thanks are due to Martin Hyland on several counts. First, for pointing out a long time ago (at the Newton Institute programme on Semantics of Computation, 1995) that we were using profunctors. Then, his Cambridge Part III lectures on category theory in the Michaelmas term 1995, emphasising ends and coends, could hardly 
have been more timely. Later the development of Section 9 (while Winskel visited Microsoft Research Cambridge) owed a great deal to discussions with Martin Hyland and John Power. John Power's course and notes on 2-categories at BRICS, Aarhus, have been very helpful, as have discussions and collaboration with Marcelo Fiore. Luca Cattani was partly supported by the EPSRC grant GR/L62290: Calculi for Interactive Systems: Theory and Experiment. A lot of the ground work was done while both authors were at BRICS, Aarhus, where it formed much of Cattani's PhD thesis [11]. 


\section{A A primer on coends and left Kan extensions}

We introduce here the key categorical notions and results that we make use of in the paper. We refer the reader to [33] and [6] for further background. ${ }^{6}$ (For the newly worked-out notions of pseudo-comonad and pseudo-distributive law we rely on $[16,46,49]$.)

Terminology and Notation: We say a category $\mathcal{C}$ is small when it is equivalent to a category whose objects and arrows form sets. We say it is locally small when for each pair of objects, $C$ and $D$, the hom-class $\mathcal{C}(C, D)$ is a set.

Correspondingly we say that a 2-category or bicategory $\mathcal{C}$ is locally small when for each pair of objects $C, D$ the category $\mathcal{C}(C, D)$ is small.

Small categories will be indicated with symbols such as $\mathbb{C}, \mathbb{D}, \mathbb{P}, \mathbb{Q}, \ldots$, while $\mathcal{C}, \mathcal{D}, \ldots$ will be used for general categories, most often locally-small.

If $\mathcal{C}$ is a category, we write $|\mathcal{C}|$ for the class of objects of $\mathcal{C}$.

We write Cat for the 2-category of small categories and CAT for the 2-category of locally small categories.

\section{A.1 Representations, universality and parametricity}

Let $\mathcal{C}$ be a category. A representation for a functor $H: \mathcal{C} \rightarrow$ Set consists of $R, \theta$, an object $R$ of $\mathcal{C}$ together with an isomorphism

$$
\theta: \mathcal{C}(R,-) \cong H
$$

A universal element of $H$ consists of $R, u$, an object $R$ of $\mathcal{C}$ and an element $u \in$ $H(R)$, such that for any object $C$ of $\mathcal{C}$ and element $x \in H(C)$ there is unique $f: R \rightarrow$ $C$ for which $x=H(f)(u)$. A representation for $H$ determines a universal element of $H$, viz. the object $R$ with the element $u=\theta_{R}\left(1_{R}\right)$. Conversely, a universal element $R, u$ of $H$ determines the representation $R, \theta$ in which the component of isomorphism $\theta$ at an object $C$ sends $f \in \mathcal{C}(R, C)$ to $\theta_{C}(f)=H f(u)$.

Parametrised representability Assume a functor $H: \mathcal{C} \times \mathcal{B} \rightarrow$ Set such that for every (parameter) $B$ an object of $\mathcal{B}$,

$$
\theta_{B}: \mathcal{C}(R(B),-) \cong H(-, B)
$$

is a representation. From the full and faithfulness of the (contravariant) Yoneda embedding, it follows that there is a unique extension of $R(-)$ to a functor $R(-)$ : $\mathcal{B} \rightarrow \mathcal{C}$ such that the isomorphism $(*)$ is natural in $B$.

A representation for a functor $H: \mathcal{C}^{o p} \rightarrow$ Set is defined dually, and parametricity follows similarly. Universal elements of such a functor have various names (universal cones or limits, universal wedges or ends, universal arrows, $\cdots$ ) according to the nature of the sets $H$ yields (cones, wedges, arrows, $\cdots$ ). Similarly, universal elements of a functor $H: \mathcal{C} \rightarrow$ Set have names (universal (co)cones or colimits, universal wedges or coends, universal arrows, $\cdots)$ according to the nature of the sets $H$ yields ((co)cones, wedges, arrows, $\cdots$ ).

\footnotetext{
${ }^{6}$ Although we shall not be so formal here the constructions on categories and functors form the basis for a term language for functors and typing judgements assigning categories as types; a correct typing judgement will ensure the functoriality of a term in its free variables. Such judgements can be accompanied by a useful catalogue of natural isomorphisms of the kind appearing here [8,7].
} 


\section{A.2 (Co)Ends and their properties}

\section{A.2.1 Dinatural transformations}

Coends and ends are generalisations of colimits and limits to functors of mixed variance. Functors of mixed variance are related by dinatural transformations.

Definition A.1 (Dinatural transformations) Let

$$
F, G: \mathcal{C}^{\text {op }} \times \mathcal{C} \rightarrow \mathcal{D}
$$

be two functors. A dinatural transformation $\alpha: F \stackrel{.}{\longrightarrow} G$ from $F$ to $G$ consists of a family of arrows $\left(\alpha_{C}: F(C, C) \rightarrow G(C, C)\right)_{C \in \mathcal{C} \mid}$, such that for every arrow of $\mathcal{C}$, $f: C \rightarrow C^{\prime}$ the following hexagonal diagram commutes:

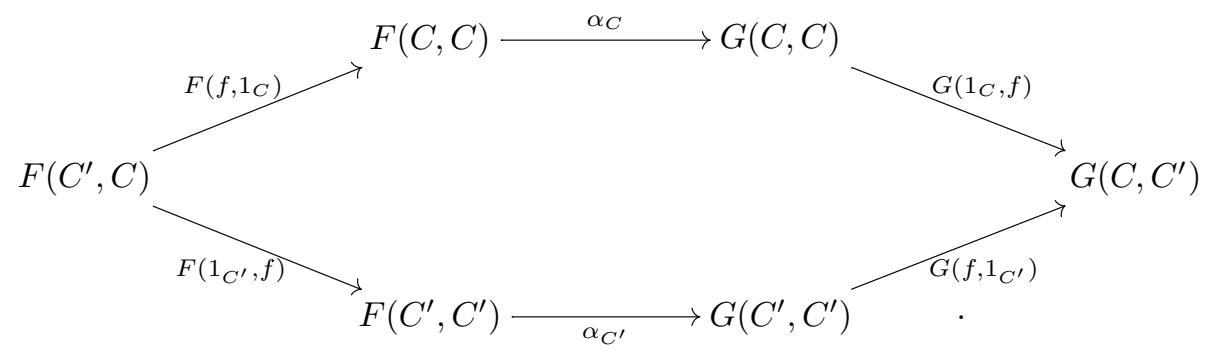

Write Dinat $(F, G)$ for the class of dinatural transformations from $F$ to $G$.

We obtain special dinatural transformations by restricting natural transformations $\beta$ in $\left[\mathcal{C}^{\text {op }} \times \mathcal{C}, \mathcal{D}\right]$ to their diagonal components, of shape $\beta_{C, C}$. Dinatural transformations do not compose in general. However, dinaturals do compose with dinaturals obtained from natural transformations. For small $\mathcal{C}$, this ensures that the $\operatorname{set} \operatorname{Dinat}(F, G)$ is functorial in $F$ and $G$, both ranging over the functor category $\left[\mathcal{C}^{\text {op }} \times \mathcal{C}, \mathcal{D}\right]$.

\section{A.2.2 Coends}

Wedges are dinatural transformations to or from a constant functor. They are thus a generalisation of cones which are natural transformations to or from a constant functor.

Notation: Any object $D$ of $\mathcal{D}$, gives rise to a constant functor, $\Delta D: \mathcal{C}^{\text {op }} \times \mathcal{C} \rightarrow \mathcal{D}$, always returning $D$ on objects and $1_{D}$ on arrows.

Definition A.2 (Wedges) Let $F: \mathcal{C}^{\mathrm{op}} \times \mathcal{C} \rightarrow \mathcal{D}$ be a functor and let $D$ be an object of $\mathcal{D}$. A wedge from $F$ to $D$ is a dinatural transformation $\alpha: F \stackrel{. \cdot}{\longrightarrow} \Delta$. In other words such a wedge consists of components $\alpha_{C}: F(C, C) \rightarrow \Delta D$, such that for any $f: C \rightarrow C^{\prime}$ the following diamond commutes:

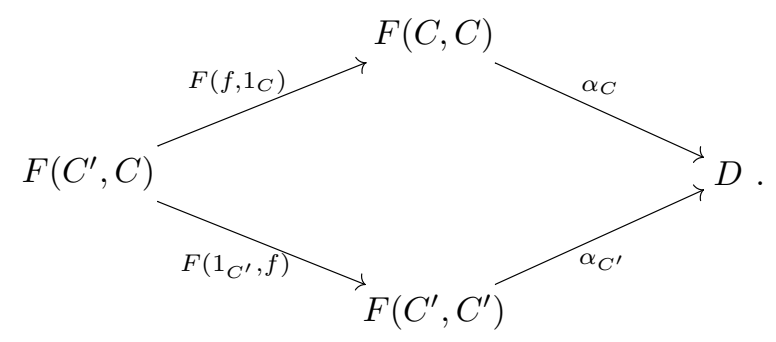


Coends are universal wedges, just as colimits are universal cones.

We can describe a coend for $F$ compactly as a representation determined by an object coend $F$ together with an isomorphism

$$
\mathcal{D}(\text { coend } F,-) \cong \operatorname{Dinat}\left(F, \Delta_{-}\right) \text {. }
$$

Equivalently we can define coends in terms of universal wedges:

Definition A.3 (Coends) $A$ coend of a functor $F: \mathbb{C}^{\mathrm{op}} \times \mathbb{C} \rightarrow \mathcal{D}$ is a universal wedge of $F$, i.e., it consists of $D_{0}, \omega$ where $D_{0}$ is an object of $\mathcal{D}$ and $\omega$ is a wedge from $F$ to $D_{0}$ such that, given any other wedge $\alpha: F \ddot{\longrightarrow} D$, there exists a unique arrow $h: D_{0} \rightarrow D$ such that $\alpha_{C}=h \omega_{C}$ for every $C \in|\mathbb{C}|$.

As usual with colimits (and limits), by abuse of language the object $D_{0}$ itself will be often called the coend of $F$, and sometimes written as coend $F$. More often though we will use the integral notation, writing

$$
\text { coend } F=\int^{C} F(C, C),
$$

always understood with respect to a particular choice of universal wedge.

Colimits as coends Colimits amount to coends of functors in which the contravariant argument is dummy. A colimit of a functor $F: \mathbb{C} \rightarrow \mathcal{D}$, can be viewed as a coend of a functor $F \pi_{2}: \mathbb{C}^{\mathrm{op}} \times \mathbb{C} \rightarrow \mathcal{D}$, where $\pi_{2}: \mathbb{C}^{\mathrm{op}} \times \mathbb{C} \rightarrow \mathbb{C}$ is the obvious projection functor. The colimit colim $F$ can be written as the coend

$$
\int^{C} F(C),
$$

in which the first dummy variable is not mentioned.

Natural transformations in $[\mathbb{C}, \mathcal{D}]$ correspond to dinatural transformations between functors in $\left[\mathbb{C}^{\mathrm{op}} \times \mathbb{C}, \mathcal{D}\right]$ in which the contravariant arguments are dummy. The characterisation of the colimit as a representation

$$
\mathcal{D}(-, \operatorname{colim} F) \cong[\mathbb{C}, \mathcal{D}](F, \Delta-),
$$

of the functor giving the set of cones from $F$ to - is a special case of the representation for coends.

Coends as colimits We can regard coends as special kinds of colimits. Assume $F: \mathbb{I}^{\mathrm{op}} \times \mathbb{I} \rightarrow \mathcal{D}$ is a functor.

We construct a category $\mathbb{I}^{\mathbb{S}}$ and a functor $d^{\mathbb{S}}: \mathbb{I}^{\mathbb{S}} \rightarrow \mathbb{I}^{\text {op }} \times \mathbb{I}$ such that $\int^{I} F(I, I) \cong \operatorname{colim}\left(F \circ d^{\mathbb{S}}\right)$.

The category $\mathbb{I}^{\mathbb{S}}$ is built from the objects and arrows in the category $\mathbb{I}$ as follows:

- objects consist of the disjoint union of the objects and arrows of I;

- arrows in addition to identity arrows we have the two arrows

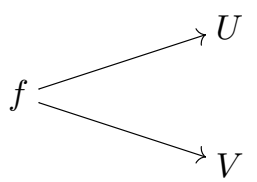

for each $f: V \rightarrow U$ in $\mathbb{I}$. 
The only composition in this category is with identities. The functor $d^{\mathbb{S}}: \mathbb{I} \rightarrow \mathbb{I}^{\text {op }} \times \mathbb{I}$ is defined as acting on objects and arrows in the following way:
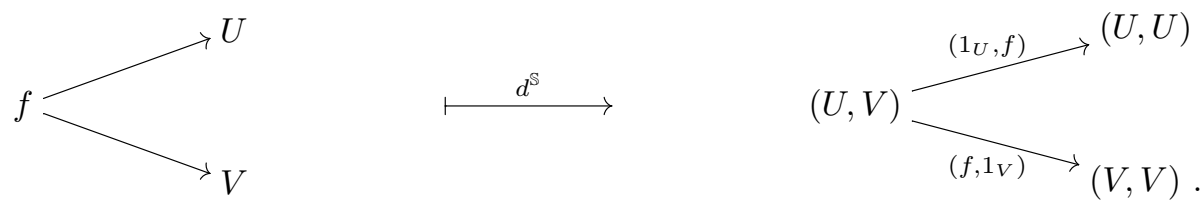

Observe that cocones in $\left[\mathbb{I}^{\mathbb{S}}, \mathcal{D}\right]\left(F \circ d^{\mathbb{S}}, \Delta D\right)$ are exactly the wedges in $\operatorname{Dinat}(F, \Delta D)$, and that a coend $\left(\int^{I} F(I, I), \omega\right)$ is a colimit for $F \circ d^{\mathbb{S}}$.

Consequently a category $\mathcal{D}$ has all small coends iff it is cocomplete, i.e. it has all small colimits.

In particular, the calculation of small coends in in Set reduces to that of a colimit in Set. The explicit construction of colimits there (see for example [33] or [6]) yields an explicit construction of coends in Set.

Proposition A.4 Let II be a small category. Let $F: \mathbb{I} \rightarrow$ Set be a functor. Then, $F$ has a colimit in Set given explicitly as the cone consisting of the set $X$ and functions $\gamma_{I}: F(I) \rightarrow X$, for $I \in|\mathbb{I}|$, described as follows. The set $X$ is the set of equivalence classes

$$
X=\sum_{I \in|\mathbb{I}|} F(I) / \sim
$$

where $\sim$ is the least equivalence relation on the set $\sum_{I \in|\mathbb{I}|} F(I) \stackrel{\text { def }}{=}\{(I, x)|I \in| \mathbb{I} \mid$ , $x \in F(I)\}$ for which

$$
(I, x) \sim(J, y) \text { if } F(f)(x)=y, \text { for some } f: I \rightarrow J \text { in } \mathbb{I} .
$$

The function $\gamma_{I}: F(I) \rightarrow X$, where $I \in|\mathbb{I}|$, takes $x \in F(I)$ to the equivalence class $\{(I, x)\}_{\sim}$.

\section{A.2.3 Parametricity for coends}

As a special case of parametrised representability, we obtain that the formation of coends maintains functoriality in parameters.

Theorem A.5 (Parametricity for coends) If $F: \mathbb{C}^{\mathrm{op}} \times \mathbb{C} \times \mathbb{B} \rightarrow \mathcal{D}$ is a functor such that for every $B \in|\mathbb{B}|$, a coend $\left(\int^{C} F(C, C, B), \omega^{B}\right)$ exists. Then, with respect to a choice of coend for each parameter $B$, the mapping

$$
B \longmapsto \int^{C} F(C, C, B)
$$

extends uniquely to a functor

$$
\int^{C} F(C, C,-): \mathbb{B} \rightarrow \mathcal{D}
$$


such that

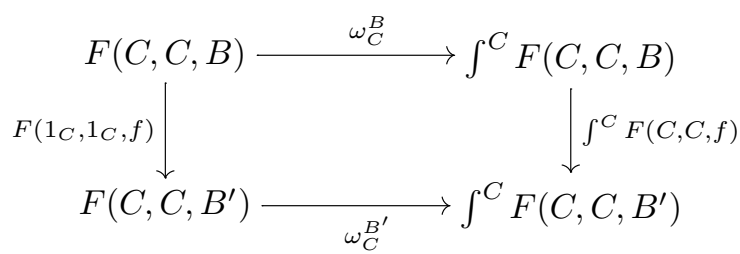

commutes for all arrows $f: B \rightarrow B^{\prime}$.

In a more compact form, the assignment $B \mapsto \int^{C} F(C, C, B)$ extends uniquely to a functor in the parameter $B$ such that the isomorphism

$$
\mathcal{D}\left(\int^{C} F(C, C, B), D\right) \cong \operatorname{Dinat}(F(-,+, B), \Delta D)
$$

natural in $D$, determined by the choice of universal wedge $\omega^{B}$, is also natural in $B$.

In line with the notation of the Theorem A.5 above, we shall write

$$
\int^{C} F(C, C, f): \int^{C} F(C, C, B) \rightarrow \int^{C} F\left(C, C, B^{\prime}\right)
$$

for the action of the functor above on the arrows $f: B \rightarrow B^{\prime}$ of $\mathbb{B}$.

In practice, parametricity often allows us to specify functors without treating objects and arrows separately. For example, with an implicit reference to parametricity, we can describe the functor above as the functor which acts so

$$
X \mapsto \int^{C} F(C, C, X)
$$

where $X$ can be understood to range over both objects and arrows. This relies on $F$ being a functor, and implicitly on a choice of coend for each object $X$.

In particular, colim $F$, which we can regard as the coend $\int^{C} F(C)$, for diagrams $F$ in $[\mathbb{C}, \mathcal{D}]$ where $\mathcal{D}$ is cocomplete, extends to a functor colim from diagrams $[\mathbb{C}, \mathcal{D}]$ to $\mathcal{D}$. Again, this assumes a choice of colimit for each diagram $F$.

\section{A.2.4 The Fubini theorem for coends}

In the manipulation of coends the interchange of "integrals" is often important, and is justified by the following theorem.

Theorem A.6 (Fubini) Given a functor $F: \mathbb{I}^{\mathrm{op}} \times \mathbb{I} \times \mathbb{J}^{\mathrm{op}} \times \mathbb{J} \rightarrow \mathcal{D}$, where $\mathcal{D}$ is a cocomplete category,

$$
\int^{I} \int^{J} F(I, I, J, J) \cong \int^{(I, J)} F(I, I, J, J) \cong \int^{J} \int^{I} F(I, I, J, J) .
$$

Moreover, the isomorphisms are natural in F.

Normally the Fubini theorem is stated in greater generality to allow for the category $\mathcal{D}$ to not have all colimits. However the simpler version suffices here. 


\section{A.2.5 Ends}

Ends are defined in a dual way to coends, as universal wedges from an object to a functor of mixed variance.

Just as colimits are special kinds of coends, we can regard limits as special ends in which the contravariant argument is dummy, and, given a functor $F: \mathcal{C} \rightarrow \mathcal{D}$ can write both $\int_{C} F(C)$ and $\lim F$ for the limit.

We can calculate ends as limits, by dualising the construction shown above for coends. In particular, we can regard an end in Set as a limit in Set from which we obtain the following explicit construction.

Proposition A.7 Let $F: \mathbb{I}^{\mathrm{op}} \times \mathbb{I} \rightarrow$ Set be a functor. Then, $F$ has an end in Set given explicitly as the wedge consisting of the set

$$
X=\left\{x \in \prod_{I \in|\mathbb{I}|} F(I, I) \mid F(I, f)\left(x_{I}\right)=F(f, J)\left(x_{J}\right) \text { for all } f: I \rightarrow J \text { in } \mathbb{I}\right\}
$$

and functions $\gamma_{I}: X \rightarrow F(I, I)$, where $I \in|\mathbb{I}|$, projecting $x$ to its component $x_{I}$.

\section{A.2.6 End and coend formulae}

Via the explicit construction of ends in Set, we can express the set of dinatural transformations between appropriate functors as an end. Letting $F, G: \mathbb{I}^{\mathrm{op}} \times \mathbb{I} \rightarrow \mathcal{D}$,

$$
\operatorname{Dinat}(F, G)=\int_{I} \mathcal{D}(F(I, I), G(I, I)) .
$$

By specialising to particular kinds of functors, we obtain an end expression for the set of natural transformations between functors $F, G: \mathbb{I} \rightarrow \mathcal{D}$ :

$$
[\mathbb{I}, \mathcal{D}](F, G)=\int_{I} \mathcal{D}(F(I), G(I)) .
$$

Recalling the compact presentation of coends and ends we obtain the following natural isomorphisms, characterising coends and ends:

$$
\mathcal{D}\left(\int^{I} F(I, I), D\right) \cong \int_{I} \mathcal{D}(F(I, I), D)
$$

natural in $D$, and

$$
\mathcal{D}\left(D, \int_{I} F(I, I)\right) \cong \int_{I} \mathcal{D}(D, F(I, I))
$$

natural in $D$.

\section{A.3 Preservation of colimits}

A functor $G: \mathcal{C} \rightarrow \mathcal{D}$ is said to preserve colimits of a diagram $d: \mathbb{I} \rightarrow \mathcal{C}$ if it sends any universal (i.e., colimiting) cone from $d$ to $X$ to a universal cone from $G \circ d$ to $G(X)$. Clearly, when $G$ preserves colimits of a diagram $d$ this entails $G(\operatorname{colim} d) \cong$ colim $G \circ d$. In general, such an isomorphism alone is not sufficient to ensure that $G$ preserves the colimit. However, with minor side conditions, naturality of the isomorphism in $d$ does ensure the colimit is preserved. Proofs of the following lemmas may be found in $[9,7]$. 
Lemma A.8 Suppose the category $\mathbb{I}$ is small and connected. Suppose categories $\mathcal{C}, \mathcal{D}$ have initial objects and all $\mathbb{I}$-colimits.

A functor $G: \mathcal{C} \rightarrow \mathcal{D}$ preserves $\mathbb{I}$-colimits iff there are isomorphisms

$$
\theta_{d}: G(\operatorname{colim} d) \cong \operatorname{colim}(G \circ d)
$$

natural in $d$ in $[\mathbb{I}, \mathcal{C}]$.

Lemma A.9 Suppose the category $\mathbb{I}$ is small. Suppose categories $\mathcal{C}, \mathcal{D}$ have all $\mathbb{I}$ colimits. Suppose that $G$ sends initial objects to initial objects.

$A$ functor $G: \mathcal{C} \rightarrow \mathcal{D}$ preserves $\mathbb{I}$-colimits iff there are isomorphisms

$$
\theta_{d}: G(\operatorname{colim} d) \cong \operatorname{colim}(G \circ d)
$$

natural in $d$ in $[\mathbb{I}, \mathcal{C}]$.

If we are interested in all colimits, we obtain the following simple statement.

Lemma A.10 Suppose categories $\mathcal{C}, \mathcal{D}$ are cocomplete.

A functor $G: \mathcal{C} \rightarrow \mathcal{D}$ preserves all colimits iff for all small $\mathbb{I}$ there are isomorphisms

$$
\theta_{d}: G(\operatorname{colim} d) \cong \operatorname{colim}(G \circ d)
$$

natural in $d$ in $[\mathbb{I}, \mathcal{C}]$.

From the Fubini theorem for coends we see a sense in which the operation of formation of coends preserves colimits. More precisely, suppose $\mathcal{D}$ is cocomplete. For any functor $F: \mathbb{J}^{\mathrm{op}} \times \mathbb{J} \rightarrow \mathcal{D}$ we can form the coend $\int^{J} F(J, J)$, and this operation is functorial in $F$. Call this resulting functor $G$-we might alternatively describe the functor $G$ using lambda notation as $\lambda F . \int^{J} F(J, J)$. Now, $G$ preserves colimits. In other words, $\int^{J} F(J, J)$ preserves colimits in the parameter $F$. By Lemma A.10, it is sufficient to observe the following chain of isomorphisms are all natural in $d: \mathbb{I} \rightarrow$ $\left[\mathbb{J}^{\mathrm{op}} \times \mathbb{J}, \mathcal{D}\right]:$

$$
\begin{aligned}
G\left(\int^{I} d(I)\right) & \cong \int^{J}\left(\int^{I} d(I)\right)(J, J) \\
& \cong \int^{J}\left(\int^{I} d(I)(J, J)\right) \\
& \cong \int^{I}\left(\int^{J} d(I)(J, J)\right) \quad \text { by Fubini, } \\
& \cong \int^{I} G(d(I)) .
\end{aligned}
$$

\section{A.4 Kan extensions and their properties}

\section{A.4.1 Left Kan extensions}

Definition A.11 (Left Kan Extensions) For functors $\mathcal{C} \stackrel{G}{\stackrel{A}{\longrightarrow}} \stackrel{F}{\longrightarrow}$, one says that a pair $K, \alpha$ is a left Kan extension of $G$ along $F$ if 
- $K: \mathcal{B} \rightarrow \mathcal{C}$ is a functor

- $\alpha: G \Rightarrow K F$ is a natural transformation satisfying the following universal property:

for every other pair $H, \beta$ with $H: \mathcal{B} \rightarrow \mathcal{C}$ and $\beta: G \Rightarrow H F$ there exists a unique $\gamma: K \Rightarrow H$ such that $\beta=\gamma F \cdot \alpha$.

By the usual abuse of language we will often call the functor $K$ the left Kan extension of $G$ along $F$ and write it as $\operatorname{Lan}_{F}(G)$.

We can summarise the data provided by the definition of left Kan extension in the diagram:

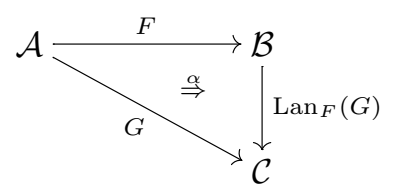

We can alternatively present such a left Kan extension as a representation, consisting of the object $\operatorname{Lan}_{F}(G)$ and an isomorphism

$$
[\mathcal{B}, \mathcal{C}]\left(\operatorname{Lan}_{F}(G),-\right) \cong[\mathcal{A}, \mathcal{C}](G,-\circ F) .
$$

Though note for $[\mathcal{A}, \mathcal{C}]$ to be locally small, so that we always obtain a set on the right, we need to assume that $\mathcal{A}$ is small $([\mathcal{B}, \mathcal{C}]$ need not be locally small).

Suppose that every $G: \mathcal{A} \rightarrow \mathcal{C}$ has a left Kan extension $\operatorname{Lan}_{F}(G), \alpha_{G}$. As a special case of parametrised representability, the operation of forming a left Kan extension on objects $G$ of $[\mathcal{A}, \mathcal{C}]$ extends uniquely to a functor $\operatorname{Lan}_{F}(-):[\mathcal{A}, \mathcal{C}] \rightarrow[\mathcal{B}, \mathcal{C}]$ such that

$$
\left(\left(\operatorname{Lan}_{F} \gamma\right) F\right) \cdot \alpha_{G}=\alpha_{G^{\prime}} \cdot \gamma
$$

for all $\gamma: G \rightarrow G^{\prime}$.

Note that the triangle above need not commute, not even up to natural isomorphism. Still, this happens in many cases of interest.

Proposition A.12 Suppose $F$ is full and faithful.

If $\left(\operatorname{Lan}_{F}(G), \alpha\right)$ exists, then $\alpha$ is a natural isomorphism.

If $\left(\operatorname{Lan}_{F}(G), \alpha\right)$ exists for all $G: \mathcal{A} \rightarrow \mathcal{C}$, then the functors $\operatorname{Lan}_{F}(-):[\mathcal{A}, \mathcal{C}] \rightarrow$ $\operatorname{Im}[\mathcal{B}, \mathcal{C}]$ and $-\circ F: \operatorname{Im}[\mathcal{B}, \mathcal{C}] \rightarrow[\mathcal{A}, \mathcal{C}]$, form an equivalence of categories between the functor category $[\mathcal{A}, \mathcal{C}]$ and $\operatorname{Im}[\mathcal{B}, \mathcal{C}]$, the full subcategory of $[\mathcal{B}, \mathcal{C}]$ consisting of functors naturally isomorphic to $\operatorname{Lan}_{F}(G)$ for some $G: \mathcal{A} \rightarrow \mathcal{C}$.

\section{A.4.2 Pointwise left Kan extensions}

As we will see shortly, if $\mathcal{C}$ is cocomplete and $\mathbb{A}$ is small, then $\operatorname{Lan}_{F}(G)$ always exists for any $F$ and $G$. The proof of this fact relies on an important general construction, that of the category of elements of a presheaf.

Definition A.13 Let $X: \mathbb{P}^{\mathrm{op}} \rightarrow$ Set be a presheaf. Define $\mathcal{E} l(X)$ to be the category consisting of

- objects pairs $(P, x)$, where $P \in|\mathbb{P}|$ and $x \in X(P)$, and 
- arrows $f:(P, x) \rightarrow\left(P^{\prime}, x^{\prime}\right)$ if $f: P \rightarrow P^{\prime}$ is an arrow of $\mathbb{P}$ and $X f\left(x^{\prime}\right)=x$.

The composition of arrows is given by the composition in $\mathbb{P}$.

The construction extends to a functor $\mathcal{E} l(-)$ from $\widehat{\mathbb{P}}$ to the category of small categories. Let $h: X \rightarrow Y$ be a map in $\widehat{\mathbb{P}}$, i.e. a natural transformation between presheaves. The naturality of $h$ ensures that we can define the functor $\mathcal{E} l(h): \mathcal{E l}(X) \rightarrow \mathcal{E} l(Y)$ by sending an object $(P, x)$ in $\mathcal{E} l(X)$ to $\left(P, h_{P}(x)\right)$ and an arrow $f:(P, x) \rightarrow\left(P^{\prime}, x^{\prime}\right)$ in $\mathcal{E} l(X)$ to the arrow $f:\left(P, h_{P}(x)\right) \rightarrow\left(P^{\prime}, h_{P}\left(x^{\prime}\right)\right)$ in $\mathcal{E} l(Y)$.

Assuming that $\mathcal{C}$ is cocomplete and $\mathcal{A}$ is small we can compute the left Kan extension $\operatorname{Lan}_{F}(G)$ "pointwise", at any object $B \in \mathcal{B}$, by taking

$$
\operatorname{Lan}_{F}(G)(B)=\operatorname{colim}(\mathcal{E l}(\mathcal{B}(F(-), B)) \stackrel{\pi}{\longrightarrow} \mathcal{A} \stackrel{G}{\longrightarrow} \mathcal{C})
$$

using the category of elements of the presheaf $\mathcal{B}(F(-), B): \mathcal{A}^{\text {op }} \rightarrow$ Set. With the understanding that $(A, x)$ ranges over this category of elements, we can abbreviate this colimit expression to

$$
\operatorname{Lan}_{F}(G)(B)=\int^{(A, x)} G A
$$

On an arrow $h: B \rightarrow B^{\prime}$, the left Kan extension produces a unique arrow $\operatorname{Lan}_{F}(G)(h)$ : $\operatorname{Lan}_{F}(G)(B) \rightarrow \operatorname{Lan}_{F}(G)\left(B^{\prime}\right)$, mediating between the two colimiting cones $\left\langle G A \stackrel{\gamma_{A, x}}{\longrightarrow}\right\rangle_{(A, x) \in|\mathcal{E} l(\mathcal{B}(F(-), B))|}$ and $\left\langle G A \stackrel{\gamma_{A, y}^{\prime}}{\longrightarrow}\right\rangle_{(A, y) \in\left|\mathcal{E} l\left(\mathcal{B}\left(F(-), B^{\prime}\right)\right)\right|}$, such that

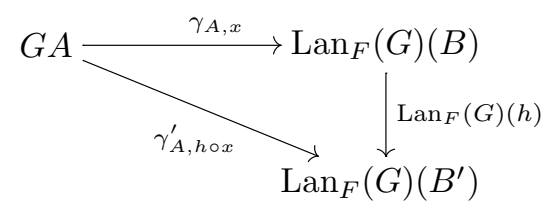

commutes for all $(A, x) \in|\mathcal{E} l(\mathcal{B}(F(-), B))|$. (See [6], Vol. 1, for a detailed proof that this construction yields a left Kan extension.)

Still assuming that $\mathcal{C}$ is cocomplete and $\mathcal{A}$ is small, there is also a useful description of (pointwise) left Kan extensions in terms of coends (Exercise 4, p. 239 of [33]):

$$
\operatorname{Lan}_{F}(G)(B) \cong \int^{a} \mathcal{B}(F(A), B) \cdot G(A),
$$

where by a copower $S . C$ is meant the coproduct $\sum_{s \in S} C$ of as many copies of $C$ as there are members of the set $S$.

\section{A.4.3 Left Kan extensions along Yoneda}

Of special interest is the case of left Kan extensions along the Yoneda embedding $\mathrm{y}_{\mathbb{P}}: \mathbb{P} \rightarrow \widehat{\mathbb{P}}$, where $\mathbb{P}$ is a small category, and and the category $\mathcal{C}$ is cocomplete:

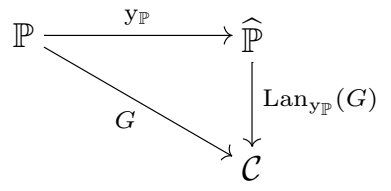


In this case $\operatorname{Lan}_{y_{\mathbb{P}}}(G)$ will always have a right adjoint $G^{*}: \mathcal{C} \rightarrow \widehat{\mathbb{P}}$ given by

$$
G^{*}(C)=\mathcal{C}(G(-), C) .
$$

When extending along Yoneda, we can use the Yoneda lemma to simplify the colimit and coend formulations of the left Kan extension given above, in Section A.4.2.

For $X$ a presheaf in $\widehat{\mathbb{P}}$,

$$
\left.\operatorname{Lan}_{\mathrm{y} \mathbb{P}}(G)(X)=\operatorname{colim}(\mathcal{E l}(X)) \stackrel{\pi}{\longrightarrow} \mathbb{P} \stackrel{G}{\longrightarrow} \mathcal{C}\right) .
$$

Let $X$ and $X^{\prime}$ be presheaves in $\widehat{\mathbb{P}}$, associated with the colimiting cones

$$
\begin{aligned}
& \left\langle G P \stackrel{\gamma_{P, x}}{\longrightarrow} \operatorname{Lan}_{\mathrm{y}_{\mathbb{P}}}(G)(X)\right\rangle_{(P, x) \in|\mathcal{E} l(X)|}, \\
& \left\langle G P \stackrel{\gamma_{P, x}^{\prime}}{\longrightarrow} \operatorname{Lan}_{\mathbb{Y}_{\mathbb{P}}}(G)\left(X^{\prime}\right)\right\rangle_{\left(P, x^{\prime}\right) \in\left|\mathcal{E} l\left(X^{\prime}\right)\right|} .
\end{aligned}
$$

For a map $h: X \rightarrow X^{\prime}$, we can define $\operatorname{Lan}_{\mathrm{yP}}(G)(h)$ to be the unique arrow in $\mathcal{C}$ such that

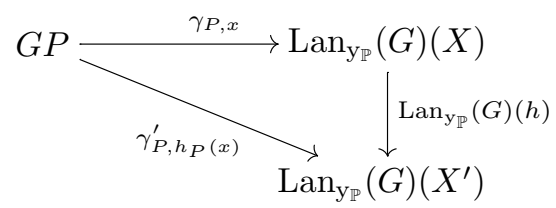

commutes for all $(P, x) \in|\mathcal{E} l(X)|$.

From the coend expression for left Kan extensions, by the Yoneda lemma,

$$
\operatorname{Lan}_{\mathrm{yP}}(G)(X) \cong \int^{P} X(P) \cdot G(P) .
$$

\section{A.4.4 The density formulae}

The left Kan extension of a Yoneda embedding along itself always exists and is naturally isomorphic to the identity. From the two ways of describing pointwise left Kan extensions we obtain two forms of the density formula. One form expresses a presheaf $X$ as a colimit of representables:

$$
X \cong \int^{(P, x)} \mathrm{y}_{\mathbb{P}}(P),
$$

where $(P, x)$ ranges over the category of elements $\mathcal{E} l(X)$. The other exhibits a presheaf as a coend:

$$
X \cong \int^{P} X(P) \cdot \mathrm{y}_{\mathbb{P}}(P) .
$$

\section{A.4.5 A factorisation lemma}

It is often useful to observe that pointwise left Kan extensions can be factored into a composition described by the following lemma. 
Lemma A.14 Let $I: \mathbb{R} \rightarrow \widehat{\mathbb{P}}$ and $G: \mathbb{R} \rightarrow \mathcal{C}$ be functors, where the category $\mathcal{C}$ is assumed cocomplete. Then,

$$
\operatorname{Lan}_{I}(G) \cong \operatorname{Lan}_{\mathrm{y}_{\mathbb{R}}}(G) \circ I^{*},
$$

where $I^{*}: \widehat{\mathbb{P}} \rightarrow \widehat{\mathbb{R}}$ is given by $I^{*}(X)=\widehat{\mathbb{P}}(I(-), X)$ :

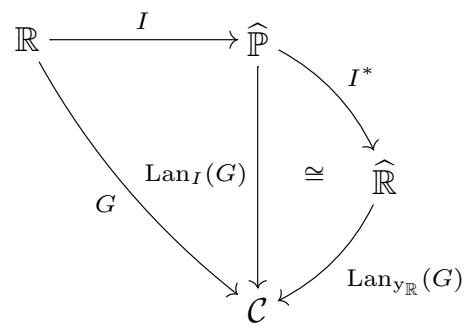

Proof: By considering the coend expressions for left Kan extensions we see that

$$
\left(\operatorname{Lan}_{I} G\right)(X) \cong \int^{R} \widehat{\mathbb{P}}(I(R), X) \cdot G R=\int^{R}\left(I^{*} X\right) R . G R \cong\left(\operatorname{Lan}_{\mathrm{y}_{\mathbb{R}}} G\right) \circ I^{*}(X)
$$

natural in $X \in \widehat{\mathbb{P}}$.

\section{A.4.6 Extensions of functors}

A functor $F: \mathbb{P} \rightarrow \mathbb{Q}$, between small categories $\mathbb{P}$ and $\mathbb{Q}$, extends to a functor

$$
\operatorname{Lan}_{\mathrm{y} \mathbb{P}}\left(\mathrm{y}_{\mathbb{Q}} \circ F\right): \widehat{\mathbb{P}} \rightarrow \widehat{\mathbb{Q}},
$$

which is traditionally denoted by $F_{\text {! }}$

As we have just seen this left Kan extension has a right adjoint $\left(\mathrm{y}_{\mathbb{Q}} \circ F\right)^{*}: \widehat{\mathbb{Q}} \rightarrow \widehat{\mathbb{P}}$, which, overloading notation, we will also write as $F^{*}$.

In fact, the functor $F^{*}$ is itself a left Kan extension along $\mathrm{y}_{\mathbb{Q}}$ of the functor $\mathbb{Q} \rightarrow \widehat{\mathbb{P}}$ taking $Q$ to the presheaf $\mathbb{Q}(F(-), Q)$. So $F^{*}$ has a right adjoint, traditionally written as $F_{*}: \widehat{\mathbb{P}} \rightarrow \widehat{\mathbb{Q}}$.

Summarising, in the special case, where $F$ is a functor from $\mathbb{P}$ to $\mathbb{Q}$ (as distinct from $\widehat{\mathbb{Q}})$ there is a triple of adjoints

$$
F_{!} \dashv F^{*} \dashv F_{*}: \widehat{\mathbb{P}} \rightarrow \widehat{\mathbb{Q}} .
$$

Further discussion on such adjoints, which form an essential geometric morphism, can be found, e.g., in [34].

\section{References}

[1] Samson Abramsky. On semantic foundations for applicative multiprogramming. In ICALP '83, Tenth Colloquium on Automata, Languages and Programming, volume 154 of Lecture Notes in Computer Science, pages 1-14. Springer-Verlag, 1983. 
[2] Jiři Adámek and Jiři Rosický. Locally Presentable and Accessible Categories, volume 189 of London Mathematical Society Lecture Notes Series. Cambridge University Press, 1994.

[3] Joyal André. Foncteurs analytiques et espaces de structures. In Proceedings of a Colloquium on Enumerative Combinatorics, volume 1234 of Lecture Notes in Mathematics. Springer-Verlag, 1985.

[4] Michael Barr. *-autonomous categories, volume 752 of Lecture Notes in Mathematics. Springer-Verlag, Berlin, 1979. With an appendix by Po Hsiang Chu.

[5] Jean Bénabou. Les distributeurs. Rapport $n^{\circ}$ 33. Seminaires de Mathématiques Pure, Institut de Mathématiques, Université Catholique de Louvain, 1973.

[6] Francis Borceux. Handbook of categorical algebra I, volume 50 of Encyclopedia of Mathematics and its Applications. Cambridge University Press, 1994.

[7] Mario Caccamo and Glynn Winskel. Lecture notes in category theory. Notes for a course given by Glynn Winskel based on Martin Hyland's Cambridge Part III lectures 1995. Available at http://www.brics.dk/ mcaccamo/, 2000.

[8] Mario Caccamo and Glynn Winskel. A higher-order calculus for categories. In Proceedings of TPHOLs 2001, volume 2152 of Lecture Notes in Computer Science. Springer-Verlag, 2001.

[9] Mario Caccamo and Glynn Winskel. Limit preservation from naturality. In Proceedings of the International Conference on Category Theory and Computer Science, CTCS'04, Electronic Notes in Theoretical Computer Science. Elsevier, 2004.

[10] Aurelio Carboni. Some free constructions in realizability and proof theory. Journal of Pure and Applied Algebra, 103(2), 1995.

[11] Gian Luca Cattani. Presheaf Models for Concurrency. PhD thesis, University of Aarhus, 1999.

[12] Gian Luca Cattani, Marcelo P. Fiore, and Glynn Winskel. A theory of recursive domains with applications to concurrency. In LICS '98, Proceedings of the Thirteenth Annual IEEE Symposium on Logic in Computer Science, pages 214-225. IEEE Computer Society Press, 1998.

[13] Gian Luca Cattani, Ian Stark, and Glynn Winskel. Presheaf models for the $\pi$ calculus. In Proceedings of the 7th International Conference on Category Theory and Computer Science, CTCS '97, number 1290 in Lecture Notes in Computer Science, pages 106-126. Springer-Verlag, 1997.

[14] Gian Luca Cattani and Glynn Winskel. Presheaf models for concurrency. In D. van Dalen and M. Bezem, editors, Computer Science Logic. 10th International Workshop, CSL '96, Annual Conference of the European Association for Computer Science Logic. Selected Papers, volume 1258 of Lecture Notes in Computer Science, pages 58-75. Springer-Verlag, 1997. 
[15] Gian Luca Cattani and Glynn Winskel. Presheaf models for CCS-like languages. Theoretical Computer Science, 300(1-3):47-89, 2003.

[16] Eugenia Cheng, Martin Hyland, and John Power. Pseudo-distributive laws. In Mathematical Foundations of Program Semantics, MFPS 19, volume 83 of Electronic Notes in Theoretical Computer Science. Elsevier, 2003.

[17] Marcelo P. Fiore, Gian Luca Cattani, and Glynn Winskel. Weak bisimulation and open maps (extended abstract). In LICS '99, Proceedings of the Fourteenth Annual IEEE Symposium on Logic in Computer Science, pages 67-76. IEEE Computer Society Press, 1999.

[18] Jean-Yves Girard. Linear logic. Theoretical Computer Science, 50(1):101, 1987.

[19] Rob van Glabbeek and Ursula Goltz. Equivalence notions for concurrent systems and refinement of actions. In Mathematical Foundations of Computer Science 1989, number 379 in Lecture Notes in Computer Science, pages 237-248. Springer-Verlag, 1989.

[20] Matthew Hennessy. A fully abstract denotational model for higher-order processes. Information and Computation, 112(1), 1994.

[21] Matthew Hennessy and Gordon D. Plotkin. Full abstraction for a simple parallel programming language. In J. Bečvár, editor, Mathematical Foundations of Computer Science (MFCS) 1979, volume 74 of Lecture Notes in Computer Science, pages 108-120, Berlin, 1979. Springer-Verlag.

[22] Thomas T. Hildebrandt. A fully abstract presheaf semantics for SCCS with finite delay. In Proceedings of the 8th International Conference on Category Theory and Computer Science, CTCS '99, ENTCS. Elsevier, 1999. To appear.

[23] Thomas T. Hildebrandt. Categorical Models for Concurrency: Independence, Fairness and Dataflow. PhD thesis, University of Aarhus, 2000.

[24] Thomas T. Hildebrandt, Prakash Panangaden, and Glynn Winskel. A relational model of non-deterministic dataflow. In D. Sangiorgi and R. de Simone, editors, Proceedings of the 9th International Conference on Concurrency Theory, CONCUR '98, volume 1466 of Lecture Notes in Computer Science, pages 613-628. Springer-Verlag, 1998.

[25] B. Jacobs and J. Rutten. A tutorial on (co)algebras and (co)induction. EATCS Bulletin, 62:222-259, 1997.

[26] Bart Jacobs. Semantics of weakening and contracion. Annals of Pure and Applied Logic, 69:73-106, 1994.

[27] André Joyal and Ieke Moerdijk. A completeness theorem for open maps. Annals of Pure and Applied Logic, 70(1):51-86, 1994.

[28] André Joyal, Mogens Nielsen, and Glynn Winskel. Bisimulation from open maps. Information and Computation, 127(2):164-185, 1996. 
[29] Gregory M. Kelly. Basic Concepts of Enriched Category Theory, volume 64 of London Mathematical Society Lecture Notes Series. Cambridge University Press, 1982.

[30] Gregory M. Kelly and M. L. Laplaza. Coherence for compact closed categories. Journal of Pure and Applied Algebra, 19:193-213, 1980.

[31] F. William Lawvere. Metric spaces, generalized logic and closed categories. Rend. Sem. Mat. Fis. Milano, 43:135-166, 1973.

[32] Daniel J. Lehmann. Categories for fixpoint semantics. In Foundations of Computer Science 17, 1976.

[33] Saunders Mac Lane. Categories for the Working Mathematician, volume 5 of Graduate Texts in Mathematics. Springer-Verlag, 1971.

[34] Saunders Mac Lane and Ieke Moerdijk. Sheaves in Geometry and Logic: A First Introduction to Topos Theory. Springer-Verlag, 1992.

[35] Michael Makkai and Robert Paré. Accessible Categories: The Foundations of Categorical Model Theory, volume 104 of Contemporary Mathematics. American Mathematical Society, 1989.

[36] Massimo Merro and Francesco Zappa Nardelli. Bisimulation Proof Methods for Mobile Ambients. In ICALP'03, Int. Colloquium on Automata Languages and Programming, number 2719 in Lecture Notes in Computer Science, pages 584598. Springer-Verlag, 2003.

[37] Robin Milner. Communication and Concurrency. International Series in Computer Science. Prentice Hall, 1989.

[38] Mikkel Nygaard. Towards an operational understanding of presheaf models. Phd progress report, BRICS, University of Aarhus, 2001.

[39] Mikkel Nygaard and Glynn Winskel. Linearity in process languages. In LICS '02, Proceedings of the Seventeenth Annual IEEE Symposium on Logic in Computer Science. IEEE Computer Society Press, 2002.

[40] Mikkel Nygaard and Glynn Winskel. Domain theory for concurrency. Theoretical Computer Science, 316(1):153-190, 2004.

[41] Robert Paré. Simply connected limits. Canadian Journal of Mathematics, XLII(4):731-746, 1990.

[42] D. M. R. Park. Concurrency and automata on infinite sequences. In Theoretical Computer Science, 5th GL-conference, volume 104 of Lecture Notes in Computer Science. Springer-Verlag, 1981.

[43] Gordon D. Plotkin. A powerdomain construction. SIAM Journal of Computation, $5(3): 456-487,1976$.

[44] Gordon. D. Plotkin. Domains. Technical report, Department of Computer Science, University of Edinburgh, 1983. Includes the "Pisa notes". 
[45] John Power. 2-Categories. BRICS Lecture Notes. University of Aarhus, 1998.

[46] John Power and Miki Tanaka. Pseudo-distributive laws and axiomatics for variable binding. Submitted for publication, 2004.

[47] R. A. G. Seely. Linear logic, *-autonomous categories and cofree algebras. In J. Gray and A. Scedrov, editors, Categories in computer science and logic, volume 92 of Contemporary Mathematics, pages 371-382. American Mathematical Society, Providence, RI, 1989.

[48] Ross Street. Fibrations in bicategories. Cahiers de Topologie et Géométrie Différentielle Catégoriques, XXI(2):111-160, 1980.

[49] Miki Tanaka. Pseudo-Distributive Laws and a Unified Framework for Variable Binding. PhD thesis, University of Edinburgh, 2004. Forthcoming.

[50] Alfred Tarski. A lattice-theoretical fixpoint theorem and its applications. Pacific Journal of Mathematics, 5, 1955.

[51] Glynn Winskel. A presheaf semantics of value-passing processes (extended abstract). In U. Montanari and V. Sassone, editors, CONCUR'96, Proceedings of the 7 th International Conference on Concurrency Theory, volume 1119 of Lecture Notes in Computer Science, pages 98-114. Springer-Verlag, 1996.

[52] Glynn Winskel. A linear metalanguage for concurrency. In Proceedings of AMAST '98, volume 1548 of Lecture Notes in Computer Science. Springer-Verlag, 1999. Invited lecture.

[53] Glynn Winskel. Linearity and nonlinearity in distributed computation. In Book on Linear Logic. Cambridge University Press, 2004. To Appear.

[54] Glynn Winskel and Mogens Nielsen. Models for concurrency. In Handbook of logic in computer science, Vol. 4, Oxford Sci. Publ., pages 1-148. Oxford Univ. Press, 1995.

[55] Glynn Winskel and Mogens Nielsen. Models for concurrency. In A. M. Pitts and P. Dybjer, editors, Semantics and Logics of Computation. Cambridge University Press, 1997.

[56] Glynn Winskel and Mogens Nielsen. Presheaves as transition systems. In D. Peled, V. Pratt, and G. Holzmann, editors, Partial Order Methods in Verification, volume 29 of DIMACS Series in Discrete Mathematics and Theoretical Computer Science, pages 129-140. AMS, 1997.

[57] Glynn Winskel and Francesco Zappa Nardelli. New-hopla-a higher order process language with name generation. In Proceedings TCS@2004, Third IFIP International Conference on Theoretical Computer Science, 2004. Kluwer, 2004. 


\section{Recent BRICS Report Series Publications}

RS-04-22 Gian Luca Cattani and Glynn Winskel. Profunctors, Open Maps and Bisimulation. October 2004. 64 pp. To appear in Mathematical Structures in Computer Science.

RS-04-21 Glynn Winskel and Francesco Zappa Nardelli. New-HOPLAA Higher-Order Process Language with Name Generation. October 2004. 38 pp.

RS-04-20 Mads Sig Ager. From Natural Semantics to Abstract Machines. October 2004. $21 \mathrm{pp}$. Presented at the International Symposium on Logic-based Program Synthesis and Transformation, LOPSTR 2004, Verona, Italy, August 26-28, 2004.

RS-04-19 Bolette Ammitzbøll Madsen and Peter Rossmanith. Maximum Exact Satisfiability: NP-completeness Proofs and Exact Algorithms. October 2004. 20 pp.

RS-04-18 Bolette Ammitzbøll Madsen. An Algorithm for Exact Satisfiability Analysed with the Number of Clauses as Parameter. September 2004. 4 pp.

RS-04-17 Mayer Goldberg. Computing Logarithms Digit-by-Digit. September 2004. 6 pp.

RS-04-16 Karl Krukow and Andrew Twigg. Distributed Approximation of Fixed-Points in Trust Structures. September 2004. 25 pp.

RS-04-15 Jesús Fernando Almansa. Full Abstraction of the UC Framework in the Probabilistic Polynomial-time Calculus ppc. August 2004.

RS-04-14 Jesper Makholm Byskov. Maker-Maker and Maker-Breaker Games are PSPACE-Complete. August 2004. 5 pp.

RS-04-13 Jens Groth and Gorm Salomonsen. Strong Privacy Protection in Electronic Voting. July 2004. 12 pp. Preliminary abstract presented at Tjoa and Wagner, editors, 13th International Workshop on Database and Expert Systems Applications, DEXA '02 Proceedings, 2002, page 436. 